\title{
SURFACE DIFFUSION STUDIES BY OPTICAL DIFFRACTION TECHNIQUES
}

\author{
XU-DONG XIAO \\ Ph.D. Thesis \\ DEPARTMENT OF PHYSICS \\ University of California \\ and \\ MATERIALS SCIENCES DIVISION \\ Lawrence Berkeley Laboratory \\ University of California \\ Berkeley, CA 94720
}

NOVEMBER 1992

This work was supported by the Director, Office of Energy Research, Office of Basic Energy Sciences, Materials Sciences Division, of the U.S. Department of Energy under Contract No. DE-AC03-76SF00098.

\section{MASTER}




\begin{abstract}
by

Xu-dong Xiao

Doctor of Philosophy in Physics

University of California at Berkeley

Professor Yuen Ron Shen, Chair
\end{abstract}

Surface Diffusion Studies By Optical Diffraction Techniques

Optical techniques have been proven to be powerful tools for surface studies. In this thesis, we have presented the newly developed techniques with either second harmonic $(\mathrm{SH})$ diffraction or linear diffraction off a monolayer adsorbate grating for surface diffusion measurement. Their development will change the situation with surface diffusion field, which has been impeded by the lack of convenient and powerful measurement methods. The anisotropy of surface diffusion of $\mathrm{CO}$ on $\mathrm{Ni}(110)$ has been used as a demonstration for the second harmonic diffraction method. The linear diffraction method, which possesses a much higher sensitivity than the SH diffraction method, has been employed to study the effect of adsorbate-adsorbate interaction on $\mathrm{CO}$ diffusion on $\mathrm{Ni}(110)$ surface. The results unambiguously showed that only the short range direct CO-CO orbital overlapping interaction influences $\mathrm{CO}$ diffusion but not the long range dipole-dipole and $\mathrm{CO}-\mathrm{Ni}-\mathrm{CO}$ interactions. Effects of impurities and defects on surface diffusion have been further explored by using the linear diffraction method on the $\mathrm{CO} / \mathrm{Ni}(110)$ system. It has been found that a few percent $\mathrm{S}$ impurity can alter the $\mathrm{CO}$ diffusion barrier height to a much higher value through changing the $\mathrm{Ni}(110)$ surface. The point defects of $\mathrm{Ni}(110)$ 
surface seem to speed up CO diffusion significantly. A mechanism with long jumps over multiple lattice distance initiated by $\mathrm{CO}$ filled vacancy has been proposed to explain the observed defect effect. 


\section{Acknowledgement}

I never could have dreamed of receiving a Ph. D. degree if there had not been political changes in China in 1979. The cultural revolution once terminated my highest level of education at middle school. I cannot deny my gratitude for a life that put me at the right time, in the right place, and with the right people.

Most of the children in my village lost their opportunities for education before entering middle school. It was my parents, who themselves did not have higher education, who encouraged and supported me to be exposed to higher and higher education. They would never have believed that in ten years, their son would leave his village forever. I want to express my warmest gratitude and love to my parents and wish someday I could be with them to truly compensate for their emotional loss due to my years abroad.

I am grateful to my advisor, Professor Yuen Ron Shen, who guided me to become an experimentalist in surface science. Without his unselfish devotion of knowledge and time it would never be possible for me to reach this final stage. His enthusiasm towards science, optimism towards difficulties, and profound intuition in physics have set a high standard that I hope to continue following in the future.

I thank all the members of the Shen group. With your help and understanding, the six and half years I have spent in graduate school was quite a pleasant journey. Marla Feller, with whom I shared an office for five years, also shared my ups and downs. Along with being a good scientist, she is also full of human emotion and enthusiasm in life. I have enjoyed her friendship and 
will cherish it for many years to come. Wei Chen, a wonderful scientist and an understanding individual, spent tremendous time with me not only to wonder why we became physicists but also to wonder what is the fate of our motherland. Chris Mullin and Dieter Wilk-- who I thank for kindly reading through my thesis and correcting a great many grammar and spelling mistakes-with your accompaniment, my final year in Berkeley has become less miserable than it would have been. Rich Superfine, Tom Moses, John O'Brien, Quan Du, and Rodney Chin, have all made their contribution to make the group a joyful environment.

I owe special thanks to Viola Vogel and Yuanlin Xie, two outstanding postdocs with whom I have worked. Viola shared her precious experience in Langmuir-Blodgett films and helped me make my first experiment a success. Yuanlin worked with me day and night in the last two years, jumping up and down around the vacuum chamber, sharing all the frustration and success. Without his collaboration and contribution in the experiment, I would never have finished the major part of this thesis.

I will always remember the invaluable advice from Rita Jones, the former group secretary. Her common sense and caring for the students in the group impressed me from the very day I joined. The day Rita left, the life seemed more boring.

I am thankful to Pete Miller, the electrician in the department. We pulled out the four heavy, about 30-years-old laser power supplies for repair nearly a hundred times in the last three years. He once joked that it was miracle that I was still alive after sticking my head underneath the laser table to disconnect and reconnect the power cables that many times. I agree with him.

Two special friends of mine outside the lab, Agnes and Felix Merz, my 
American host parents, deserve my special acknowledgement. Our friendship had started from the very day I came into this country. Wherever I go in the future, I will always think of them and remember a very true story that an American lady waited in the San Francisco airport for more than 7-hours to welcome her un-met Chinese son.

Finally, I give my deep-heart appreciation to my dear friend, Qin Xia, who has been very special to me since last year. 


\section{Table of Contents}

Acknowledgements iii

Table of Contents vi

I. General Introduction 1

$\begin{array}{ll}\text { References } & 6\end{array}$

II. Overview on Recent Development of Surface Diffusion 8

A. Introduction 8

B. Single Particle Diffusion on Surfaces 8

$\begin{array}{ll}\text { C. Chemical Surface Diffusion } & 17\end{array}$

D. Anisotropic Surface Diffusion 23

E. Effects of Defects on Surface Diffusion 25

F. Review of Surface Diffusion Techniques 30

$\begin{array}{ll}\text { References } & 35\end{array}$

III. Nonlinear and Linear Optical Diffraction Methods for Surface Diffusion Study

A. General Principles of Surface Diffusion Measurement with Optical Diffraction Methods

B. Methods to Determine Laser-Induced-Thermal-Desorption Yield

a) Reflection SHG Method $\quad 40$

b) Linear Reflectance Method 42

c) Laser Heating Model and Laser Induced 
C. Adsorbate Gratings $\quad 49$

D. Optical Diffraction as Probes of Surface Diffusion 52

a) SH Diffraction Probe 52

b) Linear Diffraction Probe $\quad 54$

E. Measuring Coverage Dependence of Diffusion 57

References $\quad 62$

Figures $\quad 63$

IV. Optical Second Harmonic Diffraction Study of Anisotropic

Surface Diffusion: CO/Ni(110)

A. Introduction $\quad 74$

B. Experiment $\quad 75$

a) Sample Preparation $\quad 75$

b) Diffusion Measurement 76

C. Relations Between Diffusion Coefficient and the

SH Diffraction Signal $\quad 78$

D. Experimental Results 81

E. Discussion 83

a) Heating Effect from the Probing Beam 83

b) Coverage Dependence of Nonlinear Susceptibility 84

c) Coverage Dependence of Diffusion Coefficient 87

d) Effects of Surface Defect 91

e) Diffusion Results 93

F. Conclusion $\quad 95$

$\begin{array}{ll}\text { References } & 97\end{array}$

$\begin{array}{ll}\text { Figures } & 98\end{array}$ 
V. Adsorbate- Adsorbate Interaction on Surface Diffusion: CO/Ni(110) 107

$\begin{array}{ll}\text { A. Introduction } & 107\end{array}$

B. Experiment Details 108

C. Results and Discussion 111

a) Measurement Accuracy 111

b) Coverage Dependent Diffusion Results 112

c) Discussion on Coverage Dependence 113

d) Comparison with Other Nickel Surfaces 115

e) Discussion on Anisotropic Activation Energy 117

f) Discussion on the Anisotropy of Preexponential Factor 119

e) Theoretical Fittings of $D(\theta) \quad 123$

$\begin{array}{ll}\text { D. Summary } & 126\end{array}$

$\begin{array}{ll}\text { Reterences } & 127\end{array}$

$\begin{array}{ll}\text { Figures } & 130\end{array}$

VI. Impurity, Defect Effects on Surface Diffusion 139

A. Introduction 139

B. Experiment 140

C. Results and Discussion 142

a) $S$ Impurity Effect 142

b) Defects Effect 144

c) Diffusion on Stepped Surface 148

$\begin{array}{ll}\text { Appendix } & 151\end{array}$

References 155

$\begin{array}{lr}\text { Figures } & 159\end{array}$

$\begin{array}{ll}\text { VII. Future Prospects } & 163\end{array}$ 


\section{General Introduction}

Surface science has been progressed enormously in the last two decades. A variety of surfaces have been investigated. In particular, many aspects of crystalline surfaces such as surface relaxation, surface reconstruction ${ }^{1-8}$, kinetics of adsorption and desorption of foreign particles ${ }^{9-17}$, and chemical reactions on surfaces ${ }^{18-23}$ have been intensively studied with a number of powerful techniques. Considerable activity in surface diffusion has also been undertaken in the last 10 years ${ }^{24-26}$. In contrast to the other subfields of surface science, however, surface diffusion is less explored and less understood.

Surface diffusion is a subject that studies stochastic motion of adparticles on surfaces. As is well kncwri, surfaces have two dimensional periodic structures and a particle, aiom or molecule, adsorbed on a surface sees a periodic potential. Most of the time the adparticle resides at a local minimum of the potential -- the adsorption site. Occasionally this adparticle jumps from one adsorption site to another in a random walk fashion through the interaction with the suostrate phonons and electrons without leaving the surface. The adparticle's random motion is usually characterized by a quantity loosely called the surface diffusion coefficient.

There are two distinctive classes of surface diffusion. The first one is concerned with a single adparticle random motion and is described by tracer diffusion coefficient $\mathrm{D}^{*}$. The second one is concerned with random motion of a macroscopic number of adparticles and is described by chemical diffusion coefficient $D^{24}$. The first one is the limit of the second at zero coverage. Much effort has been devoted to tracer diffusion by field ion microscopy (FIM) and 
other techniques ${ }^{26}$. Theoretical studies of tracer diffusion are coming into maturity as well. On the other hand, chemical diffusion study has just been started, especially in theory. The existing experimental techniques for chemical surface diffusion study are limited one way or another and a consistent picture of chemical surface diffusion has not been formed yet.

Apart from the fundamental interests of searching for surface diffusion mechanisms and measuring surface potential corrugations, surface diffusion is practically important for surface catalysis, crystal growth, and material fabrication. For example, the hydrogenation reaction of $\mathrm{CO}(\mathrm{a})+\mathrm{H}(\mathrm{a})-->\mathrm{CH}_{4}(\mathrm{~g})$ $+\mathrm{CO}_{2}(\mathrm{~g})$ on catalysts such as $\mathrm{Ni}^{27}$ relies on the mobilities of these two reactants and are likely controlled by their surface diffusions. Therefore, surface diffusion measurements for single species system as well as for mixed species system are of primary importance in understanding the reaction mechanism.

In this thesis we will first briefly review the status of the surface diffusion theory for both tracer diffusion and chemical diffusion. Despite some interesting features revealed by the theories, most of these theories are phenomenological in treating the interactions between the adparticle and the substrate as well as the interactions among the adparticles. There, we will see what still needs to be done in order to have a better understanding of surface diffusion. The experimental techniques of surface diffusion measurements will be summarized in the same chapter (chapter II) with a discussion on the limitations of each method. To overcome the limitations of these existing techniques, we have devoted our effort in developing new surface diffusion measurement techniques in the past few years and have successfully applied the new techniques to study anisotropy, coverage dependence, and impurity and defect effects of surface diffusion for $\mathrm{CO}$ of $\mathrm{Ni}(110)$.

The new techniques we have developed are based on the following 
principles. First, an adsorbate grating is created by laser induced thermal desorption (LITD) with two laser beams interfering at an adsorbate-covered solid surface. With this initial coverage profile, diffraction (linear or nonlinear) off the grating of a probing laser beam is then used to monitor the time evolution of the coverage profile. The adsorbate grating is expected to get smeared out by surface diffusion and the diffraction signal to decay correspondingly. From the decay time constant of the diffraction signal the diffusion coefficient of the adsorbate can be deduced. The details of these techniques with both $\mathrm{SH}$ diffraction and linear diffraction probes will be described in chapter III. These methods bear a number of very attractive features. First, they involve a simple one-dim insinnal diffusion process for which the data analysis is relatively straightiorimerd. Second, by properly orienting the grating, the diffusion coefficient along any direction on the surface can be directly measured. This makes the anisotropic diffusion measurement very easy. Third, as an optical method, the technique can be applied to a wide variety of adsorbates on any substrate. Fourth, with a tunable probe beam selectively probing particular species of adsorbates, surface diffusion of individual components of a mixed adsorbate system can be monitored. This allows the study of influence of surface diffusion on surface reactions such as catalysis. Fifth, the dynamic range can be extremely wide, ranging from $10^{-6}$ to $10^{-15} \mathrm{~cm}^{2} / \mathrm{s} a \mathrm{c}$ for the diffusion coefficient. Finally, the technique can be used to study other forms of surface diffusion like diffusion of electronic or vibrational excitations.

Surface second harmonic generation (SHG) as a versatile tool for surface and interface studies has been reviewed by a number of authors ${ }^{28,29}$ and has been documented in detail in a number of thesis from the Shen group $^{30,31}$. The surface specificity of $\mathrm{SH}$ process allowed us to develop a 
simple, straightforward diffraction scheme off an adsorbate monclayer grating for surface diffusion study ${ }^{31,32}$. In chapter $u$, the anisotropic surface diffusion of $\mathrm{CO} / \mathrm{Ni}(110)$ will be presented and a number of related issues will be discussed.

Despite its initial success, the SH diffraction technique still has a few limitations. The intrinsic weak response of the $\mathrm{SH}$ process prevents us from studying coverage dependence in surface diffusion. The polarization modulated linear diffraction scheme we have developed has lifted these limitations and exploited the full advantages of the optical diffraction technique for surface diffusion study. The coverage dependent diffusion study for CO/Ni(110) will be presented in chapter V. It has been found that only the short range CO-CO direct interaction influences the diffusion of $\mathrm{CO}$ on $\mathrm{Ni}(110)$, while the long range dipole-dipole interaction and $\mathrm{CO}-\mathrm{Ni}-\mathrm{CO}$ interaction do not.

There exists the question how impurities and defects could affect surface diffusion. In many surface reactions it has been found that impurities and defects can play critical roles. Is this also true for surface diffusion? If so, what are the mechanisms? We have answered these questions to a certain extent by studying $\mathrm{CO}$ diffusion on $\mathrm{S}$ contaminated $\mathrm{Ni}(110)$ surfaces and $\mathrm{Ar}^{+}$sputtered Ni(110) surfaces. The results will be presented in chapter VI. The S impurity has been found to be able to modify substantially the $\mathrm{Ni}(110)$ surface even at very low densities and thus after the $\mathrm{CO}$ diffusion activation energy. The defects of $\mathrm{Ni}(110)$ surface introduced by $\mathrm{Ar}^{+}$sputtering can also influence $\mathrm{CO}$ diffusion. However, the effect is quite different as the diffusion speed actually increases with increase of defects. Mechanism based on long jumps over multiple lattice distance assisted by $\mathrm{CO}$ filled vacancy has been proposed to interpret the the observation.

Future prospects will be discussed in Chapter VII. A few important issues 
in surface diffusion will be addressed and some interesting experiments will be proposed. 


\section{References}

1 A. Thomy, X. Dual and J. Regnier, Surf. Sci. Rep. 1, 1(1981).

2 A. Kahn, Surf. Sci. Rep. 3, 193(1983).

${ }^{3}$ I. P. Ipatova and Yu. E. KitseV, Prog. Surf. Sci. 18, 189(1985).

${ }^{4}$ H. Ohtani, C.-T. Kao, M. A. Hove and G. A. Somorjai, Prog. Surf. Sci. 23, 155(1986).

${ }^{5}$ R. Feidenhans'l, Surf. Sci. Rep. 10, 105(1989).

${ }^{6}$ S. Pick, Surf. Sci. Rep. 12, $G_{9}(1991)$.

${ }^{7}$ B. N. J. Person, Surf. Sci. Rep. 15, 1(1992).

8 J. P. LaFemina, Surf. Sci. Rep. 16, 133(1992).

9 J. Czarniecki and M. Jaroniec, Surf. Sci. Rep. 3, 301(1983).

${ }^{10}$ G. Comsa and R. David, Surf. Sci. Rep. 5, 145(1985).

${ }^{11}$ E. Shustorovich, Surf. Sci. Rep. 6, 1(1986).

12 H. P. Bonzel, Surf. Sci. Rep. 8, 43(1987).

${ }^{13}$ T. Aruga and Y. Murata, Prog. Surf. Sci. 31, 61(1989).

14 J. K. Norskov, Rep. Prog. Phys. 53, 1253(1990).

${ }^{15}$ V. P. Zhdanov, Surf. Sci. Rep. 12, 183(1991).

${ }^{16}$ R. D. Ramsier and J. T. Yates, Jr., Surf. Sci. Rep. 12, 243(1991).

17 S. J. Lombardo and A. T. Bell, Surf. Sci. Rep. 13, 1(1991).

${ }^{18}$ M. P. Kiskinova, Surf. Sci. Rep. 8, 359(1988).

${ }^{19}$ L. K. Doraiswamy, Prog. Surf. Sci. 37, 1(1991).

${ }^{20}$ H. F. Winters and J. W. Coburn, Surf. Sci. Rep. 14, 161(1992).

21 J. Nishizawa and H. Sakuraba, Surt. Sci. Rep. 15, 137(1992).

22 E. A. Colbourn, Surf. Sci. Rep. 15, 281(1992).

${ }^{23}$ L. J. Richter and R. R. Cavanagh, Prog. Surf. Sci. 39, 155(1992). 
${ }^{24}$ Robert Gomer, Rep. Prog. Phys. 53, 917(1990).

25 A. Kapoor, R. T. Yang, and C. Wong, Catal. Rev.-Sci. Eng., 31, 129(1989);

A. G. Naumovets and Yu. S. Vedula, Surt. Sci. Rep. 4, 365(1985);

Gert Ehrlich and Kaj Stolt, Ann. Rev. Phys. Chem. 31, 603(1980).

${ }^{26}$ T. T. Tsong, Surf. Sci. Rep. 8, 127(1988); Prog. Surf. Sci. 10, 165(1980).

${ }^{27}$ G. A. Somorjai, Chemistry in Two Dimensions: Surfaces, (Cornell University Press, Ithaca New York, 1981).

${ }^{28}$ Y. R. Shen, Annu. Rev. Phys. Chem. 40, 327(1989).

${ }^{29}$ G. L. Richmond, J. M. Robinson and V. L. Shannon, Prog. Surf. Sci. 28, 1(1988).

${ }^{30}$ Tony F. Heinz, Ph. D. Thesis, Berkeley, 1984;

Harry K. Tom, Ph. D. Thesis, Berkeley, 1985;

Wei Chen, Ph. D. Thesis, Berkeley, 1990;

Marla B. Feller, Ph. D. thesis, Berkeley, 1991.

31 X. D. Zhu, Ph. D. Thesis, Berkeley, 1989.

${ }^{32}$ X. D. Zhu, Th. Raising and Y. R. Shen, Phys. Rev. Lett. 61, 2883(1988). 


\section{Overview on Recent Development of Surface Diffusion}

\section{A. Introduction}

As pointed out in Chapter I, surface diffusion is of interest from both basic and technological view points. Theoretical studies of surface diffusion have undergone substantial progress in the past 10 years $^{1-4}$. Analytical and numerical tools have been developed for this purpose. The results obtained so far will be briefly reviewed in the next few sections. The surface diffusion theory can be classified into two categories: one deals with tracer diffusion of a single adparticle; the other deals with chemical diffusion of a large number of adparticles among which interactions may not be negligible.

Experimentally, quite a few techniques have been developed in the last two decades. Using these methods a 'arge base of data on surface diffusion has been accumulated. However, all these techniques have limitations which have prohibited the building of a consistent picture for surface diffusion. Most of these techniques will be discussed in section F. Similar to the theoretical situation, the experimental studies iall into two classes as well. In the first class, tracer diffusion of single adsorbed particles is monitored. In the second class, chemical diffusion of a large number of adparticles is measured.

\section{B. Single Particle Diffusion on Surfaces}

An understanding of single particle diffusion on a surface from first principles was achieved early in this century ${ }^{5,6}$. This was a first step towards 
understanding more complicated diffusion. A microscopic picture of the motion of an adsorbed particle, atom or molecule, can be established in terms of interaction between the adparticle and the substrate atoms. The substrate applies not only a two-dimensional periodic static potential but also a random force to the adsorbed particle. It is this dynamic random force that initiates a jump of the adparticle and then damps the motion ${ }^{5,7}$. The origin of the random force is the thermal vibration of the substrate atoms and the excitation of the substrate electrons ${ }^{7}$. Because of the randomness of the dynamic interaction between the adparticle and the substrate, the motion of the adparticle is essentially Brownian. To understand the nature of surface diffusion it is critical to first understand the nature of these interactions.

In order to describe the motion of the adparticle, models of the dynamic interaction with the substrate (or, the heat bath) must be employed. The very first systematic treatment was due to Kramers ${ }^{5}$, who described the random force from the surroundings (heat bath) by a fluctuating force $\xi(t)$ with a white noise spectrum and a linear damping force $-M \eta \dot{x}$, with $\eta$ as the friction coefficient and $\dot{x}$ as the velocity. The fluctuating force obeys the fluctuation-dissipation theorem

$$
\begin{aligned}
& <\xi(t)>=0, \\
& <\xi(t) \xi(s)>=2 M \eta k_{B} T \delta(t-s),
\end{aligned}
$$

where $M$ is the mass of the adparticle, $k_{B}$ the Boltzmann constant, and $T$ the absolute temperature. With the above mentioned forces, the equation of motion for the adsorbed particle is then given by the Langevin equation

$$
M \ddot{x}=-\nabla U(x)-M m \dot{x}+\xi(t),
$$


where $U(x)$ is the static potential. Unlike classical mechanics, the motion of the adparticle in this situation is not determined since the fluctuating force has only statistical meaning. Starting from the Langevin equation, Kramers was able to derive the Klein-Kramers equation ${ }^{5,6}$

$$
\frac{\partial \rho(x, v, t)}{\partial t}=\left[-\frac{\partial}{\partial x} v+\frac{\partial}{\partial v} \frac{U^{\prime}(x)+M \eta v}{M}+\frac{\eta k T \partial^{2}}{M} \partial v^{2}\right] \rho(x, v, t),
$$

with $\rho(x, v, t)$ as the probability density for finding the adparticle at $x$ with velocity $v$ at time $t$, and $U^{\prime}(x)$ the derivative of the static potential with respect to $x$. There is no general solution to this equation. To treat the jumping rate problem for a particle from one potential well to another, Kramers simplified this equation with further assumptions and then deduced the jump rate in terms of the vibrational frequency $\omega$ of the adparticle and the friction coefficient $\eta$. In the case of strong viscosity, the effect of the Brownian forces on the velocity of the particle is much larger than that of the static force $-\nabla U(x)$, and a Maxwell velocity distribution can be established in a time scale shorter than $1 / \omega$. Thus, we have

$$
\rho(x, v, t)=\sigma(x, t) \exp \left(-m v^{2} / 2 k T\right) .
$$

This probability density leads to a jump rate $e^{5,6}$

$$
\begin{array}{ll}
k=\frac{\omega \omega *}{2 \pi \eta} \exp \left(-E_{a} / k_{B} T\right), & \text { for } \eta>\omega^{*} \\
k=\frac{\omega}{2 \pi} \exp \left(-E_{a} / k_{B} T\right), & \text { for } \omega k_{B} T / 2 \pi E_{a}<\eta<\omega^{*}
\end{array}
$$

where $\omega=(U " / M)^{1 / 2}$ evaluated at the potential minimum is the angular 
frequency of small oscillations of an adsorbed particle near the equilibrium position, $\omega^{*}=(-U / M)^{1 / 2}$ evaluated at the saddle point is the angular frequency characterizing the potential barrier ( $U$ " being the second order derivative), and $E_{a}$, the barrier height, is the energy difference between the saddle point and the bottom of the potential well. In the case of small viscosity, the Brownian forces cause only a small change of the energy during a period of oscillation of the particle in a potential well and thus the probability density can be written as a function of energy and time, $\rho(x, v, t)=\rho(E, t)$, and a jump rate

$$
k=\eta \frac{E_{a}}{k_{B} T} \exp \left(-E_{a} / k_{B} T\right), \quad \text { for } \eta<\omega k_{B} T / 2 \pi E_{a}
$$

can be deduced. In the moderate friction regime, the result Eq. (4b) is identical to that from absolute rate theory (or transition state theory, TST, see discussion below), in which the friction coefficient is irrelevant. However, in both weak and strong friction regimes, the diffusion rate is significantly different from that of the absolute rate theory. It is also clear that the Kramers theory only provides a way for calculating the jump rate rather than the diffusion coefficient. Further assumptions on jumping mechanisms are required in order to find the diffusion coefficient. For instance, in most surface diffusion cases, the elemental jumps of the adparticle are to the adjacent site. Therefore the diffusion coefficient is given by 8

$$
D=\frac{1}{4} k\left\langle i^{2}\right\rangle
$$

where $\left\langle\left.\right|^{2}\right\rangle$ is the mean square jump length corresponding to the lattice distance. 
Kramers theory deals with the jump rate of a structureless adparticle in one dimension. For surface diffusion, the adparticle can have many degrees of freedom of motion. How to couple these other degrees of freedom into the jump rate is a difficult problem. As a result, the extension of Kramers theory into multidimensions is very complicated ${ }^{6}$. Opposite to this, the transition state theory provides a simple, direct way to solve the problem to some extent.

The basic assumptions in the transition state theory (TST) are the following: (1) the adparticles are in equilibrium with the static substrate potential well at every point; (2)only adparticles with energy higher than the potential barrier will jump to the next site. Under these assumptions, finding the jump rate becomes a simple statistical problem. With $\mathrm{Z}_{0}$ as the partition function of the adparticle at the bottom of the potential well and $Z^{\neq}$as the partition function at the saddle point, excluding the diffusion coordinate, the jump rate can be expressed as 6

$$
k_{T S T}=\frac{k_{B} T}{h} \frac{Z^{\neq}}{Z_{0}} \exp \left(-E_{a} / k_{B} T\right) .
$$

For an adparticle without internal degrees of freedom and in one dimension, it is easy to verify that Eq. (6) gives result (4b).

The degrees of freedom other than the diffusion coordinate can affect the jump rate if the corresponding partition functions at the bottom of the well and at the saddle point are not the same. For example, for an adparticle diffusing on a surface, the second translational degree of freedom will come into the two partition functions differently. Furthermore, if the adparticle is a molecule, its bending modes can affect the jump rate through the two corresponding partition 
functions as well.

Despite the success of the Kramers' theory, it is still an oversimplified model. The actual dynamic interaction between the adparticle and the substrate is far more complicated ${ }^{7}$. Furthermore, the phenomenological parameter $\eta$ should be calculable from the microscopic origin of the interaction. Several authors have derived expressions for this parameter $\eta$ by considering the dynamic interaction between the adparticle and the substrate due to both electronic excitations and lattice vibrations ${ }^{9}$. In general, since the electrons are much lighter than the adparticle and consequently their fluctuating motions are fast with respect to the motion of the adparticle. Treating the forces from the electrons as a Brownian-like force as in the Kramers model is a very good approximation. However, fluctuations of the substrate atoms are on a time scale comparable to or even longer than the motion of the adparticle, making the assumption of the Kramers model for the dynamic interaction very questionable.

Goran Wahnstrom ${ }^{7}$ and S. C. Ying ${ }^{10}$ independently developed theories to account for the above effect. In their theories, information about the dynamic interactions is contained in a so called memory function, from which the diffusion coefficient $D$ can be deduced. The details of these theories must be referred to the original papers. The important result is that in most cases, a spatially dependent friction coefficient $\eta(\mathbf{r})$, in contrast to Kramers' constant friction coefficient, must be used in the Langevin equation in order to describe the diffusive motion of the adparticle well. In the case of light adparticles; even a position dependent friction coefficient is no longer appropriate to describe the adparticle's motion and a full account for the couplings between the adparticle and the substrate must be taken. This is because that the motion of the substrate atoms is slower than the motion of the adparticle, the forces applied to 
the adparticle by the substrate atoms can no longer be described as Brownian force.

Aside from the analytical description of adparticle diffusion, molecular dynamics simulation has been extensively used and has proved to be a powerful tool to study surface diffusion of a single adsorbate on crystalline surfaces ${ }^{11}$. The principles for applying molecular dynamics simulation in surface diffusion are the following. The diffusion coefficient $D^{*}$ is defined in the random walk picture by ${ }^{11}$

$$
D^{*}=\lim _{t \rightarrow \infty} \frac{\leq \Delta R^{2}(t)>}{4 t}
$$

with $<\Delta f^{2}(t)>$ as the mean square displacement of the adparticle. This formula can be rewritten in terms of the velocity correlation

$$
D^{*}=\int_{0}^{\infty} d t \frac{1}{2}\langle v(0) \cdot v(t)>
$$

With either Lennard-Jones or Morse potential describing the interacion between the adparticle and substrate atoms as well as between substrate atoms, the system can be thermalized at finite temperature and then the trajectories of the adparticle followed so that the mean square displacement, or the velocity correlation, can be calculated and the diffusion coefficient found. The diffusion activation energy and the pre-exponential factor can be subsequently determined from an Arrhenius fit.

The trajectory tracing procedure in the molecular dynamics simulation is very transparent and many interesting features of surface diffusion have been 
found with this method. For instance, concerted motion with the adparticle exchanging position with a substrate atom ${ }^{12}$, correlated successive hoppings $^{12,13}$, multiple-lattice distance hoppings ${ }^{12,13}$, and recrossing of the saddle point have been observed. These provide essential information for understanding the diffusion mechanisms.

Unfortunately, molecular dynamics simulation is not adequate for diffusion studies at all temperatures for most given systems. Especially, at low temperatures, the adparticle resides at its local minimum energy site for such a long time that a jumping event is very rare. Directly simulating such a system for surface diffusion takes an unreasonably long time and it is impossible in practice. An alternative method to solve this problem is to incorporate a molecular dynamics simulation into the transition state theory (TST).

Formalisms for this purpose have been developed ${ }^{14}$. The key point in this approach is to factor the jump rate constant into two parts: (1) an equilibrium factor which is simply the transition state theory (TST) rate constant. The rareevent nature of the process is included in this factor; (2) a dynamic correction factor, $f_{d}(i->j)$, which accounts for the fact that the flux crossing the dividing surface that separates the initial and final sites of a jump in (1) contains spurious crossings. The spurious crossings do not correspond to true sitechange event. The jump rate from site $i$ to site $j$ itius can be expressed as

$$
k_{i->j}=k_{T S T}{ }_{d}^{(i->j)}
$$

The dynamic correction factor $f_{d}$ (i->j) can be evaluated by following the trajectories of the adparticle in a short-time regime in the molecular dynamics simulation. To illustrate this point further, we look at two different time scales; $\tau_{\text {corr }}$, which is the average time it takes an adparticle to thermalize with its 
surroundings; and $\tau_{\mathrm{rxn}}$, which is the average time between two reactive successive crossings. Since the residence time for the adparticle in its equilibrium site is usually very long in the low temperature regime, it is generally true that $\tau_{\text {corr }} \ll \tau_{\text {rxn }}$. With the dynamic phenomena such as saddle point recrossing, multiple-lattice distance jumping, and correlated successive jumping all happening in the time scale $\tau_{\text {corr }}$, it is easy to see that only the dynamic information on this time scale is needed for calculating the dynamic correction factors and this dynamic information can be obtained by setting the initial conditions properly in a simulation.

Once the rates $k_{i->j}$ are known, the diffusion coefficient is then given by

$$
D=\frac{1}{4} \sum_{j \neq i} k_{i->j} l_{i j}^{2}
$$

where multiple-lattice distance jumping has been explicitly included. It is found in the low temperature regime that the TST theory is quite accurate. However, in the high temperature regime, multiple lattice distance jump rate $k_{i->j}(|i-j|>1)$ is appreciable and contributes to the diffusion coefficient signaificantly ${ }^{11}$.

Recently, a molecular dynamics simulation was applied to the surface diffusion of $\mathrm{CO}$ on $\mathrm{Ni}(11 \mathrm{i})^{15}$. It was found that $\mathrm{CO}$ is subjected to a rather weak dissipative force or the $\mathrm{Ni}(111)$ surface, so that correlated jumps and multiplelattice distance jumps are rather common (with a probability of 0.5 ). The internal degrees of freedom of the $\mathrm{CO}$ molecule is also important. In particular, the coupling between the diffusion coordinate and the bending mode is very strong. The potential seen by $\mathrm{CO}$ can be significantly modified when the molecule rocks uphill and downhill. 
If only the static interaction is concerned, more advanced methods such as the ab initio, local density function (LDF) method can be used to calculate the diffusion activation energy and the lowest energy path. An example is Al/Al $(100)^{16}$, in which concerted motion is found by the LDF method.

\section{Chemical Surface Diffusion}

In the tracer surface diffusion case, where only motion of noninteracting individual atom or molecule is involved, random walk models can be applied. The diffusion coefficient can be directly related to the microscopic quantities such as mean square displacement and jump rate. However, in the chemical surface diffusion case, where a macroscopic number of interacting atoms or molecules are involved, the diffusion can no longer be defined by simple microscopic quantities ${ }^{1}$. The counterpart of the tracer diffusion coefficient $D^{*}$ in the chemical diffusion case is the chemical surface diffusion coefficient $D$, defined by Fick's first law',

$$
\mathbf{J}=-\stackrel{\leftrightarrow}{\mathbf{D}} \cdot \nabla C
$$

where $\mathbf{J}$ is the adparticle flux and $\mathrm{C}$ the adparticle density. Here, we explicitly indicate the tensor characteristic of the surface diffusion coefficient by $\stackrel{\leftrightarrow}{\mathbf{D}}$. In the isotropic case, the diffusion tensor reduces to a scalar and

$$
\mathbf{J}=-\mathrm{D} \nabla \mathbf{C}
$$

In a macroscopic system, equilibrating adatoms or molecules on a 
surface is a response to the gradient in chemical potential $\mu$ rather than the gradient of the concentration. Therefore, a more appropriate way to express the flux is ${ }^{17}$

$$
\begin{aligned}
\mathrm{J} & =-\mathrm{L}_{\mathrm{T}} \nabla_{\mu} \\
& =-\frac{\mathrm{L}_{T}}{\mathrm{~N}_{S}}\left(\frac{\partial \mu}{\partial \theta}\right)_{\Gamma} \nabla C,
\end{aligned}
$$

where $L_{T}$ is a constant. The variable $C$ in the chemical potential has been replaced by coverage $\theta$ througn the relation $C=N_{S} \theta$, with $N_{S}$ being the density of available adsorption sites on the surface. We have

$$
\begin{aligned}
D & =\frac{L_{T}}{N_{S}}\left(\frac{\partial \mu}{\partial \theta}\right) T \\
& =\frac{1}{4} a^{2} \Gamma(\theta)\left(\frac{\partial\left(\mu / k_{B} T\right)}{\partial \ln \theta}\right) T .
\end{aligned}
$$

The second step here is to introduce a new parameter $\Gamma(\theta)=\frac{4}{a^{2}} \frac{k_{B} T}{\theta} \frac{L_{T}}{N_{S}}$, a quantity called effective jump frequency in analogy to the jump rate $k$ in tracer dittusion case. The thermodynamic factor $\left(\frac{\partial(\mu / k T)}{\partial \ln \theta}\right)_{\Gamma}$, historicaily from Darken equation ${ }^{1}$, is related to the mean value and the mean square fluctuation of the number of adparticles ${ }^{1,17}$ : 


$$
\left(\frac{\partial(\mu / k T)}{\partial \ln \theta}\right)_{T}=\frac{<N>}{\langle\Delta N\rangle^{2}}
$$

The effect of this thermodynamic factor on diffusion coefficient has been discussed rather in detail by Ref.[17] and can be calculated once the thermodynamic property is known. However, Eq. (13) only defines the effective jump frequency $\Gamma(\theta)$ phenomenologically and does not provide a way for calculating it.

Zhdanov has provided a better picture for the effective jump frequency $\Gamma(\theta)$ in the framework of lattice-gas model ${ }^{18}$. Consider a system with adsorioate interaction which affects the energy potential at adsorption site by $\varepsilon_{\mathrm{i}}$ and the saddle point by $\varepsilon^{*}$ under a specific environment configuration (the arrangement of the rest adparticles) marked by $i$. Assuming a one dimensional coverage gradient exists, the flux of particles from row 1 to row 2 is then given by

$$
J_{12}=N_{l} \frac{1}{4} v \exp \left(-E_{a} / k_{B} T\right) \sum_{i} P_{A 0} \exp \left(-\left(\varepsilon_{i}^{*}-\varepsilon_{i}\right) / k_{B} T\right)
$$

where $N_{\ell}=1 / a$ is the number of sites on a unit length and $v$ the effective vibrational frequency of the adparticle at the bottom of the potential well at $\theta=0$. $E_{a}$ is the diffusion activation energy at $\theta->0 . \quad P_{A O} i$ is the probability that a site in the first row is occupied and the nearest site in the second row is empty, with the environment configuration marked by $i$. It can be further expressed as

$$
P_{A O i}=P_{00 i} \exp \left[\left(\mu_{1}-\varepsilon_{i}\right) / k_{B} T\right]
$$


where $\mu_{1}$ is the chemical potential in row 1 and $P_{00 i}$ is the probability that a pair of the nearest neighbor sites, one in row 1 and the other in row 2, are empty, with an environment configuration marked by $i$. Similarly the flux of particles from row 2 to row 1 can be found. With $S$ defined as

$$
S=\sum_{i} P_{00 i} \exp \left(-\varepsilon_{i}^{*} / k_{B} T\right)
$$

the net flux from row 1 to row 2 is given by

$$
\begin{aligned}
J & =J_{12}-J_{2} 1 \\
& =N_{2} \frac{1}{4} v \exp \left(-E_{a} / k_{B} T\right) S\left[\exp \left(\mu_{1} / k_{B} T\right)-\exp \left(\mu_{2} / k_{B} T\right)\right] \\
& =-\frac{1}{4} v a^{2} \exp \left(-E_{a} / k_{B} T\right) \exp \left(\mu(\theta) / k_{B} T\right) \frac{1}{\theta}\left(\frac{\partial\left(\mu / k_{B} T\right)}{\partial \ln \theta}\right) T \nabla C,
\end{aligned}
$$

where the difference in the square bracket has been replaced by an appropriate derivative. Up to this point, the effective jump frequency $\Gamma(\theta)$ has been related to fundamental quantities through $S$ and chemical potential $\mu$ as

$$
\Gamma(\theta)=v \exp \left(-E_{a} / k_{B} T\right) \operatorname{Sexp}\left(\mu(\theta) / k_{B} T\right) / \theta .
$$

and can be calculated once those quantities are known. The diffusion coefficient $D$ as a function of coverage is given by

$$
D(\theta)=\frac{1}{4} v a^{2} \exp \left(-E_{a} / k_{B} T\right) \operatorname{Sexp}\left(\mu(\theta) / k_{B} T\right) \frac{1}{\theta}\left(\frac{\partial\left(\mu / k_{B} T\right)}{\partial \ln \theta}\right) r
$$


As an example, we consider the mean field approximation with a square lattice in the lattice gas frame and restrict ourselves to the nearest-neighbor interaction. We write down ${ }^{19,19 a}$

$$
\begin{aligned}
& \exp (\mu(\theta) / k T)=\frac{\theta}{1-\theta} \exp (4 \varepsilon \theta / k T), \\
& S=(1-\theta)^{2} \exp (-6 \varepsilon * \theta / k T) .
\end{aligned}
$$

The detail derivation of Eq. (21a) can be found in Ref. [19a]. To derive Eq. (21b) one simply has used the fact that the probability of finding an empty site is (1 $\theta)$. The coverage dependent diffusion coefficient is thus obtained as

$$
\begin{aligned}
D(\theta)= & \frac{1}{4} a^{2} v \exp \left(-E_{a} / k T\right) \\
& \times(1-\theta) \exp (4 \varepsilon \theta / k T) \exp (-6 \varepsilon * \theta / k T) \\
& \times\left\{\frac{1}{1-\theta}+4 \varepsilon \theta / k T\right\}
\end{aligned}
$$

The physical meaning of this formula is worth exploring. The first line is the diffusion coefficient for a single adparticle. The factor $(1-\theta)$ is the site blocking effect in a random distribution configuration of adsorbates. The two exponential factors are the effect on the effective jump frequency from the lateral interactions of the adsorbates. The last factor is the thermodynamic factor.

For repulsive nearest-neighbor interaction, $\varepsilon$ is positive. If there is no effect on the saddle point $\left(\varepsilon^{*}=0\right)$, the repulsive interaction not only lowers the jumping barrier so as to increase the effective jumping frequency, but also increases the thermodynamic factor. Therefore, the diffusion coefficient 
increases with coverage until a very high coverage where the blocking effect (1 - $\theta)$ dominates, and then it decreases. If the nearest-neighbor interaction is attractive, the diffusion coefficient will monotonically decrease to 0 . Certainly the interactions between the adsorbates can affect the saddle point as well. As a further complication, the lateral interaction can be long range (e.g., dipole interactions) so that one adparticle can interact with many other adparticles which are far away. Many body effects such as adsorbate-substrate-adsorbate interaction can be important as well. It is this interaction that provides attraction between adsorbates in most cases ${ }^{20}$. None of these have been fully explored and they still comprise the most challenging problems in surface diffusion.

More sophisticated models, such as the quasi-chemical approximation (QCA), have been used to calculate the effective jump frequency $\Gamma(\theta)$ to investigate the effect of adsorbate interactions on surface diffusion. Nearestneighbor interactions ${ }^{17,18,21}$, next nearest-neighbor interactions and interactions at saddle points ${ }^{18}$ have all been considered. The qualitative behavior of the diffusion coefficient as a function of coverage is similar to that in the mean field approximation. In the same frame, surface diffusion in the case with adsorbate-induced surface reconstruction ${ }^{22}$ and coadsorbate surface diffusion ${ }^{23}$ have also been studied.

As we discussed in the previous section, the dynamic interaction of adparticles with the substrate is the driving force for surface diffusion. It is also true that other adparticles can exert dynamic fluctuating forces on the adparticle under consideration. How this dynamic interaction affects surface diffusion and depends on coverage are not included in the lattice gas model and has never been studied. It is certain that the other adparticles on the surface can also 
serve as a heat bath for the specific adparticle in a similar way as the substrate. Therefore, including only the static interaction from other adsorbates as in the lattice gas model is insufficient. The importance of such effects may require a first principle calculation to reveal.

The blocking effect can be more complicated in the case with ordered domain formation. If attractive interactions exist so that the molecules like to form two dimensional islands, the blocking effect is apparently larger than what the $(1-\theta)$ factor can account for. This factor $(1-\theta)$ can only describe a random adsorbates distribution case. Therefore, correct description of the site blocking effect is not a simple matter.

In principle, molecular dynamics simulation can be used in chemical diffusion study as well. Unfortunately, the large number of molecules involved in the problem makes it too time consuming and practically impossible. Instead, Monte Carlo simulations have been applied in many cases ${ }^{17,24,25}$ including ordered surface layers ${ }^{25}$. The lateral interactions used in these Monte Carlo simulations are basically the lattice gas type. Mostly, only the first nearestneighbor and second nearest-neighbor interactions have been considered. The results are qualitatively the same as those from the analytical study with the lattice gas model.

\section{Anisotropic Surface Diffusion}

For surfaces with crystallographic unequivalent directions, surface diffusions along them are expected to be different. In the extreme case, adparticles on such surfaces can preferentially hop in one direction, leading to 
one-dimensional diffusion. In most cases, diffusion on such surfaces can be a competition between the two directions. In understanding the details of diffusion mechanisms, measurement of the anisotropic surface diffusion is important. One natural question is how many measurements are needed in order to describe a two dimensional diffusion completely.

As we know, surface diffusion is mathematically described by a secondrank tensor $\stackrel{\leftrightarrow}{\mathbf{D}}$, which has four elements. In general, the diffusion tensor can be diagonalized so that there are only two independent elements that should be determined ${ }^{26}$. To see this diagonalization, let's break the diffusion tensor into a symmetric and an antisymmetric part as

$$
\overleftrightarrow{\mathbf{D}}=\left(\begin{array}{ll}
D_{11} & D_{12} \\
D_{21} & D_{22}
\end{array}\right)=\mathbf{D}^{+}+\mathbf{D}^{-}
$$

Substituting this into Fick's second law of diffusion

$$
\frac{\partial \mathrm{C}}{\partial \mathrm{t}}=\nabla \cdot(\mathbf{D} \cdot \nabla \mathrm{C})
$$

we obtain

$$
\frac{\partial C}{\partial t}=\nabla \cdot\left(D^{+} \cdot \nabla C\right)+\nabla \cdot\left(D^{-} \cdot \nabla C\right)
$$

For a diffusion coefficient which can be approximated by a constant in the specific coverage range, the second term is given by 


$$
\nabla \cdot D^{*} \cdot \nabla C=D_{12}^{-} \frac{\partial^{2} C}{\partial x_{1} \partial x_{2}}+D_{21}^{-} \frac{\partial^{2} C^{2}}{\partial x_{2} \partial x_{1}}
$$

which vanishes since $D_{12}^{-}=-D_{21}^{-}$. Therefore, the antisymmetric part of the diffusion coefficient has no physical importance in the real diffusion measurement and the surface diffusion can be fully characterized by a symmetric diffusion tensor, which can always be diagonalized. To further prove $D_{12}^{-}=-D_{21}^{-}=0$, general Onsager reciprocity relations must be applied ${ }^{26 a}$.

From the above argument, it is clear that anisotropic surface diffusion in general needs only two independent measurements in order to fully specify the diffusion properties.

Anisotropic surface diffusion has been rarely investigated theoretically. For single adparticle diffusion, can the diffusion along a principal axis be treated as a one dimensional diffusion or not? Is there a coupling between diffusion along the two principal directions? These questions have not yet been answered.

In the case of chemical surface diffusion, if the adsorbate-adsorbate interaction is anisotropic, how can this anisotropy be incorporated into the lattice gas model or other theories? This needs to be addressed before we can fully understand the diffusion anisotropy.

\section{E. Effects of Defects on Surface Diffusion}

An important question in surface diffusion is how the surface defects 
influence the adparticle diffusion. In tracer surface diffusiun, the measurement techniques are microscopic and the defect problem has been simply avoided. It is however possible that a defect can cause some modification in the vicinity of the defect site, thus affecting diffusion of the adparticle in that vicinity. Such problems unfortunately have remained unexplored. On a different note, diffusion of single adparticles in a disordered lattice system has been extensively studied by random walk models with pre-specified distributions of the jump rate ${ }^{27}$.

In the chemical surface diffusion cases, a large crystal surface is usually used and the effect of surface defects could be important. With some density of point defects, the dynamic interaction of the adparticle with the substrate, or the friction force, may be strongly altered but the static potential may remain approximately unchanged. As a result, the diffusion measurement of such a system may provide an intrinsic activation energy but not a reliable preexponential factor. How to evaluate this statement remains as an unsolved interesting problem. On the other hand, theoretical studies of chemical dittusion on an inhomogeneous surface in the framework of lattice gas model have appeared recently ${ }^{28}$.

The effect of steps on surface diffusion is easier to analyze. In the case of low step densities, we can assume that surface diffusion consists of two independent parts, one on terraces and the other on steps. No interactions between steps or between steps and terraces have to be introduced in the first order approximation. Consider a case with unidirectional steps. For diffusion parallel to steps, the diffusion coefficient is simply the sum of two diffusion coefficients with the proper weighting: 


$$
D=\eta_{s} D_{s}+\eta_{t} D_{t}
$$

where $D_{s}$ and $D_{t}$ are diffusion coefficients for diffusion on terraces and on steps, respectively, and $\eta_{s}, \eta_{t}$ are the area percentages of steps and terraces on the surface. For diffusion perpendicular to the steps, the diffusion coefficient can be obtained by summing up the diffusion time for adparticles to cross a terrace and to traverse an adjacent step. With $D_{s}^{\prime}$ denoting the diffusion coefficient when the duration time of the adparticle on terraces is negligible and $D_{t}$ denoting the diffusion coefficient when the duration time of the adparticle on steps is negligible respectively, the diffusion coefficient in general is given by

$$
\frac{1}{D}=\frac{1}{D_{s}^{\prime}}+\frac{1}{D_{t}^{\prime}}
$$

We have used the relation that the diffusion coefficient is inversely proportional to the diffusion time to obtain Eq.(27). Equations (26) and (27) indicate that the diffusion coefficients in the case with unidirectional steps can be constructed as either in series or in parallel, analogous to the case in electric circuits.

Recently, it was pointed out that the steps can dominate surface diffusion even if the step density is low ${ }^{19,29}$. The important issue here is the difference between the terrace diffusion activation energy and the step diffusion activation energy. If the time a molecule is trapped in the potential well associated with a step is long compared to the time it spends to cross a terrace, the surface diffusion perpendicular to the steps will be controlled by the steps. The condition for this to occur is the following:

$$
\gamma=N^{2} \exp \left[\left(E_{\text {diff }}(\text { terrace })-E_{\text {diff }}(\text { step })\right) / k_{B} T\right] \ll 1,
$$


where $\mathrm{N}$ is the number of rows of atoms in a terrace. For obtaining Eq. (28), we have assumed that the pre-exponential factors for the elementary jumping processes of an atom or molecule from one site to another on a terrace and across a step are the same. It is clear that the maximum $\mathbf{N}$ for step dominated diffusion is closely related to the difference between the terrace diffusion activation energy and the step diffusion activation energy. If the assumption on the pre-exponential factors is lifted, a modification on the step density will result in the step controlled diffusion condition.

In addition to controlling surface diffusion, steps can change the anisotropy of surface diffusion. In general, steps make an angle with respect to the principal axes, e.g., a surface miscut is in some arbitrary direction with respect to the principal axes. Although microscopically steps are developed to be parallel to either of the two principal axes, we assume that these step-kinklike small segments of steps can be considered to form macroscopic steps at an angle with respect to the surface principal axes and a unique diffusion activation energy across these macroscopic steps exists. Bearing this assumption in mind, we are going to construct the diffusion tensor on a surface with such unidirectional steps.

The intrinsic surface diffusion tensor for a surface without steps is given by

$$
\overleftrightarrow{\mathbf{D}}=\left(\begin{array}{cc}
\mathrm{D}_{1} & 0 \\
0 & \mathrm{D}_{2}
\end{array}\right)
$$

with the diagonal elements along the principal axes of the surface. Denoting the 
angle between the steps and the first principal axis as $\phi_{0}$, and using the coordinate frame with the two axes along or perpendicular to the steps, the intrinsic diffusion tensor is transformed to

$$
\stackrel{\leftrightarrow}{\mathbf{D}}^{\prime}=\left(\begin{array}{cc}
\mathrm{D}_{1} \cos ^{2} \phi+\mathrm{D}_{2} \sin ^{2} \phi & \left(\mathrm{D}_{1}-\mathrm{D}_{2}\right) \sin \phi \cos \phi \\
\left(\mathrm{D}_{1}-\mathrm{D}_{2}\right) \sin \phi \cos \phi & \mathrm{D}_{1} \sin ^{2} \phi+\mathrm{D}_{2} \cos ^{2} \phi
\end{array}\right)
$$

The effect of steps on the diffusion tensor now only shows up in the diagonal elements but not in the off-diagonal elements. The diffusion tensor element in the direction perpendicular to the steps should be replaced by $D_{1}{ }^{\prime}$ with (see Eq. (27))

$$
\frac{1}{D_{1}^{\prime}}=\frac{1}{D_{1} \cos ^{2} \phi+D_{2} \sin ^{2} \phi}+\frac{1}{D_{s}}
$$

where $D_{S}^{\prime}$ is the diffusion coefficient across steps. The diffusion tensor element in the direction parallel to the step is replaced by (səe Eq. (23))

$$
D_{2}^{\prime}=\eta_{t}\left(D_{1} \sin ^{2} \phi+D_{2} \cos ^{2} \phi\right)+\eta_{s} D_{s}
$$

With the two off-diagonal elements remaining the same as in Eq. (30), the new diffusion tensor is also symmetric. Generally, it can not be diagonalized in any of the two coordinate frames mentioned above. However, the general property of a symmetric tensor allows it to be diagonalization in some other coordinate frame. 


\section{F. Review of Surface Diffusion Techniques}

The techniques that are available for surface diffusion measurements have been reviewed ${ }^{1}$. Generally, these techniques are divided into two different categories: those that measure the tracer diffusion and those that measure the chemical diffusion. Detail description of these techniques can be found in Gomer's review article. In the following I will briefly describe these techniques and point out their limitations.

The techniques to measure tracer diffusion are the field ion microscopy (FIM) and scanning tunneling microscopy (STM). In the FIM technique the adatom and the atoms in the first layer of the substrate can be imaged by imaging gases, usually $\mathrm{He}$ or $\mathrm{Ne}$, so that the random motion of the adatom can be directly observed ${ }^{30}$. The very high electric field $(\sim 1 V / \AA)$ near the tip surface (substrate) involved in the image process and the field-induced stress can alter the surface potential in some cases so that the diffusion is not purely intrinsic. Also, the tip materials are usually restricted to refractory metals and the adparticles in most cases are limited to metal atoms.

In comparison, the recently developed scanning tunneling microscope, or STM, has several adyantages in measuring surface diffusion ${ }^{31}$. There, a tip with nominally one or a few atoms at the end is used to image the surface structure. The principle of operation is the following. When the tip is close enough to the surface (a few $\AA$ ), the electrons can tunnel through the vacuum gap between the tip and the surface with an applied bias voltage. As the tip moves around at a constant height the local electron density of the substrate can be mapped. An adatom on the surface can change the local electron density enough for the recognition of the existence of the adatom. This image 
process clearly allows direct observation of the adatom diffusion. Other mode of operation exist as well. The electric field required for STM is at least an order of magnitude weaker than that for FIM. Furthermore, the only requirement on the substrate surface is a large enough conductance. Therefore, adatom diffusion on semiconductors can also be studied. On the other hand, for some metal substrates with electrons highly delocalized, the atomic resolution might be lost and diffusion of adparticles on such surfaces are not observable. As a whole, STM is a more versatile technique for tracer surface diffusion study. Because STM is able to image a large area $(1 \mu \times 1 \mu)$, diffusion of a large number of adparticles (submonolayer) has been investigated with this method as well ${ }^{31}$.

Most of the diffusion measurement techniques were developed to measure chemical surface diffusion. All but one (FEM, see below) require an initial coverage gradient profile. The technique that has been employed to measure surface diffusion for many systems is the fluctuation-correlation field emission microscopy (FEM) ${ }^{32}$. The principle of this method is that the number of electrons emitted from a tip to a screen under a high electric field depends on the adsorbate coverage on the tip because the work function of the tip depends on the adsorbate coverage. With the numbers of atoms or molecules fluctuating in a restricted area, the emitted electron current fluctuates. This fluctuation can then be related to the surface diffusion coefficient through the current fluctuation-correlation function. Since no initial coverage gradient profile is required in this method, it is basically an equilibrium method and very suitable for study of coverage dependence in surface diffusion. The drawbacks are mainly from the restriction on the tip material, similar to those menticned for FIM. Therefore, the systems commonly studied with this method are molecules or atoms on various tungsten and nickel surfaces ${ }^{1}$. 
Another technique that has gained popularity in recent years is the so called "hole burning" LITU method ${ }^{33}$, in which a laser pulse is first used to desorb adsorbed molecules or atoms from the surface to create a hole in the adsorbate layer, and then a second laser pulse is subsequentially applied to probe the hole with a mass spectrometer to measure the amount of molecules that backfilled the hole from the surroundings via surface diffusion. This second laser pulse can be applied at different delayed times so that a refilling curve can be measured, and subsequently the diffusion coefficient can be deduced. The advantage of this method is its simplicity and its applicability to a large family of adsorbates and substrates. The difficulties are as follows. First, with lateral adsorbat-adsorbate interactions the diffusion coefficient is not simply related to the refilling rate. A theoretical expression of the refilling that takes these lateral interactions into account does not exist ${ }^{34}$. Second, the application of multiple laser pulses can damage the substrate surface ${ }^{35}$. Third, the coverage dependence is difficult to measure in a well sharacterized manner. Lastly, the information on diffusion anisotropy is difficult to obtain. Recently, a modified scheme of LITD has been proposed oy King's group ${ }^{36}$ where a step-like initial coverage profile is formed with laser desorption and the time evolved profile is also detected by laser desorption. By applying Boltzmann-Matano analysis, the coverage dependence of surface diffusion of $H, D / R h(111)$ has been investigated. The disadvantage of this method is the experimental complexity and the low spatial resolution ( $250 \mu \mathrm{m})$. Only relatively fast diffusion can be measured, which can be a problem if the desorption rate competes with the diffusion rate.

Many other methods have been developed to measure surface diffusion. Usually, these methods monitor a time-dependent change of a coverage profile with a certain spatial resolution. These methods include the work function 
change method, the field emission shadowing method, and the electron beam scanning method ${ }^{1}$. The work function change method has a low spatial resolution, typically in the $\sim 100 \mu \mathrm{m}$ regime. The energy resolution $(\sim 0.1 \mathrm{eV})$ associated with this method limits the systems it can measure. Typically, a work function change of $\sim 1 \mathrm{eV}$ upon adsorption is required for good measurements. The field emission shadowing method has the same limitations of FIM and FEM. The Auger electron scanning method has high spatial resolution ( $50 n \AA$ ) , but is limited to species it can detect (for example, ilydrogen is not observable) and to substrates with high enough electric conductance in order to avoid the surface charging effect. All these methods share a common disadvantage, which is the difficulty in creating a well tharacterized initial coverage profile. Therefore, they have not been very popular and only limited systems have been studied.

Methods based on FTIR and NMR have been used to study surface cififusion in some systems. The FTIR method ${ }^{37}$ relies on the spectral difference for adpa tizles on different sites, and therefore it can not be used to study diffusion on a horiogeneous surface with single type sites. The NMR method ${ }^{38}$ uses the property that an adparticle has different resonant frequencies in different environments. A spectral density analysis of the NMR spectra then allows the deduction of the diffusion coefficient. This method is very limited because of its complicated data analysis and low signal-to-noise ratio.

Helium scattering has been applied to study surface diffusion as well ${ }^{39}$. The diffusion coefficient is related to the scattering peak width. The analysis is very model dependent and therefore the results are only qualitatively meaningful.

One important aspect of surface diffusion is the directional dependence 
of the diffusion coefficient on a crystalline surface. Only some of the above methods can be used to study surface diffusion anisotropy. The difficulties lies mostly in preparing the initial coverage profile. In this thesis I will describe optical diffraction methods for surface diffusion studies. The newly developed methods can overcome many of the shortcomings mentioned above. In short, the methods allow one to study surface diffusion of any adsorbate on any substrate with coverage dependence and anisotropy of surface diffusion measured in simple manner. 


\section{References:}

1 Robert Gomer, Rep. Prog. Phys. 53, 917(1990).

${ }^{2}$ A. Kapoor, R. T. Yang, and C. Wong, Catal. Rev.-Sci. Eng., 31, 129(1989).

${ }^{3}$ A. G. Naumovets and Yu. S. Vedula, Surf. Sci. Rep. 4, 365(1985).

${ }^{4}$ Gert Ehrlich and Kaj Stolt, Ann. Rev. Phys. Chem. 31, 603(1980).

5 H. A. Kramers, Physica 7, 284(1940); S. Chandrasekher, Rev. Modern Phys. 15, 1(1943).

${ }^{6}$ Peter Hanggi, Peter Talkneer, and Michal Borkovec, Rev. Modern Phys. 62, 251(1990); V. P. Zhdanov, Surt. Sci. Rep. 12, 183(1991).

7 Goran Wahnstrom, Surf. Sci. 159, 311(1985); Surf. Sci. 164, 449(1985); J. Chem. Phys. 84,5931(1986); Phys. Rev. B 33, 1020(1985).

${ }^{8}$ V.P Zhdanov, Surf. Sci. 214, 289(1989)

${ }^{9}$ W. L. Schaich, J. Chem. Phys.60, 1087(1974); A. Blandin, A. Nourtier and D. W. Hone, J. Physique 37, 369(1976); C. Caroli, B. Roulet and D. Sain-James, Phys. Rev. B 18, 545(1978); B. Hellsing and M. Persson, Phys. Scripta 29, 360(1984); P. Minnhagen, J. Phys C 15, 2293(1982).

${ }^{10}$ S. C. Ying, Phys. Rev.B 41, 7068(1990).

11 J. D. Doll and A. F. Voter, Ann, Rev. Phys. Chem. 38, 413(1987).

12 G. De Lorenzi and G. Jacucci, Surf. Sci. 116, 391(1982).

${ }^{13}$ M. R. Mruzik and G. M. Pound, J. Phys. F:Metal Phys. 11, 1403(1981); J. D. Doll and H. K. McDowell, J. Chem. Phys. 77, 479(1982); John C. Tully, George H. Gilmer and Mary Shrgard, J. Chem. Phys. 71, 1630(1979). 14 J. C. Keck, Discuss. Faraday Soc. 33, 173(1962); D. Chandler, J. Chem. Phys. 68, 2959(1978); J. A. Montgomery, Jr., D. Chandler, and B. J. Berne, J. Chem. Phys. 70, 4056(1979); J. L. Skinner and P. G. Wolynes, J. Chem. 
Phys. 69, 2143(1978); 72, 4913(1980); Arthur F. Voter and Jimmie D. Doll, J. Chem. Phys. 82, 80(1984).

D. J. Doren, APS meeting Bulletin 37, 527(1992); K. D. Dobbs and D. J.

Doren, preprint (submitted to J. Chem. Phys.).

${ }^{16}$ P. J. Feibelman, Phys. Rev. Lett. 65, 729(1990).

17 David A. Reed and Gert Ehrlich, Surf. Sci. 102, 588(1981); 105, 603(1981).

18 V. P. Zhdanov, Surf. Sci. Lett. 149, L13(1985).

${ }^{19}$ V. P. Zhdanov, Phys. Lett. A 137, 409(1989).

${ }^{19 a}$ Follower, Statistical Thermodynamics, (Camnridge Univ. Press, 1939).

20 Yu. K. Toubin, Prog. in Surt. Sci. 34, 1(1991).

${ }^{21}$ V. P. Zhdanov, Surf. Sci. 257, 63(1991).

22 V. P. Zhdanov, Langmuir 5, 1044(1989).

${ }^{23}$ V. P. Zhdanov, Phys. Lett. A 137, 225(1989); Surf. Sci. 194, 1(1988); V.

Pereyra, G. Zgrablish, and V. P. Zhdanov, Langmuir 6, 691(1990).

24 Michael Bowker and David A. King, Surf. Sci. 71, 583(1978); 72, 208(1978).

25 G. E. Murch, Philos. mag. A 43 871(1981); Xue-Pei Jiang and Horia Metiu, J. Chem. Phys. 88, 1891(1988).

${ }^{26}$ W. Jost, Diffusion in Solids, Liquids, Gases, (Academic Press, New York, 1960).

$26 a$ L. Onsager, Phys. Rev. 37, 405(1931); 38, 2265(1931).

27 J. W. Haus and K. W. Kehr, Phys. Rep. 150, 264(1987).

${ }^{28}$ C. H. Mak, H. C. Anderson, S. M. George, J. Chem. Phys. 88, 4052(1988); V. Pereyra, G. Zgrablich, and V. P. Zhdanov, Langmuir 6, 691(1990); F. Bulnes, J. L. Riccardo, G. Zgrablich and V. Pereyra, Surf. Sci. 260, 304(1992).

${ }^{29}$ XU-dong Xiao, X. D. Zhu, W. Daum and Y. R. Shen, accepted for publication by Phys. Rev. B.

${ }^{30}$ G. Ehrlich and F. G. Hudda, J. Chem. Phys. 44, 1039(1966); G. Ehrlich, J. 
Chem. Phys. 44, 1050(1966); G. Ehrlich, Surf. Sci. 63,422(1977); G. L. Kellogg, T. T. Tsong, and P. Cowan, Surf. Sci. 70, 485(1978); T. Tsong, Rep. Prog. Phys. 51, 759(1988); G. L. Kellogg and P. J. Feibelman, Phys. Rev. Lett. 64, 3143(1990); G. L. Kellogg, Phys. Rev. Lett. 67, 216(1991); T. T. Tsong and C. Chen, Phys. Rev. B 43, 2007(1991).

${ }^{31}$ G. Binnig, H. Fuchs, and E. Stoll, Surf. Sci. 169, L295(1986); R. C. Jaklevic and L. Elie, Phys. Rev. Lett. 60, 120(1988); M. G. Lagally, Y.-W. Mo, R. Kariotis, B. S. Swartzentruber, and M. B. Webb, in Kinetics of Ordering and Growth at surfaces, edited by M. G. Lagally (Plenum, New York, 1990), p.145; H. B. Elswijk, A. Hoeven, E. J. van Loenen, and Dijkamp, J. Vac. Sci. Technol. B 9, 451(1991); Y.-W. Mo, J. Kleiner, M. B. Webb, and M. G. Lagally, Phys. Rev. Lett. 66 1998(1991); R. M. Feenstra, A. J. Slavin, G. A. Held, and M. A. Lutz, Phys. Rev. Lett. 66, 3257(1991); Eric Ganz, Silva K. Theiss, Ing-Shouh Hwang, and Jene Golovchenko, Phys. Rev. Lett. 68, 1567(1992). R. Gomer, Surf. Sci. 38, 373(1973); J. R. Chen, and R. Gomer, Surf. Sci. 81, 589(1979); R. Gomer, Vacuum 33, 537(1983).

R. Viswanathan, D. R. Burgess,Jr., P. C. Stair, and E. Weitz, J. Vac. Sci. Technol. 20, 605(1982); R. B. Hall, and A. M. DeSantolo, Surf. Sci. 137, 421(1984); S. M. George, A. M. DeSantolo, and R. B. Hall, Surf. Sci. 159, L425(1985); E. G. Seebauer and L. D. Schmidt, Chem. Phys. Lett. 123, 129(1986); C. H. Mak, J. L. Brand, A. A. Deckert, and S. M. George, J. Chem. Phys. 85, 1676(1986); C. H. Mak, J. L. Brand, B. G. Koehler, and S. M. George, Surf. Sci. 191, 108(1987); D. R. Mullins, B. Roop, S. A. Costello, and J. M. White, Surf. Sci. 186, 67(1987); C. H. Mak, and S. M. George, Surf. Sci. 172, 509(1986).

${ }^{34}$ M. C. Tringides, Surf. Sci. 204, 345(1988).

35 I. N. Rusell, Jr., R. B. Hall, Surf. Sci. 203, L642(1988). 
S. S. Mann, T. Seto, C. J. Barnes, and D. A. King, Surf. Sci. 261, 155(1992).

37 J. E. Reutt-Robey, D. J. Dore, Y. J. Chabal, and S. B. Christman, Phys. Rev. Lett. 61, 2778(1988); S. S. Iyer, T. F. Heinz, and M. M. T. Loy, J. Vac. Sci. \& Technol. B 5, 709(1987).

38 Po-Kang Wang, Jean-Phillipe Ansermet, C. P. Slichter, and J. H. Sinfelt, Phys. Rev. Lett. 55, 2731(1985); Po-Kang Wang, Jean-Phillipe Ansermet, S.

L. Rudaz, Z. Wang, S. E. Shore, C. P. Slichter, and J. H. Sinfelt, Science 234, 35(1986); S. E. Shore, Jean-Phillipe Ansermet, C. P. Slichter, and J. H.

Sinfelt, Phys. Rev. Lett. 58, 953(1987); Mamfred Riehl-Chudoba, U.

Memmert, and D. Fick, Surf. Sci. 245, 180(1991).

39 J. W. M. Frenken, B. J. Hinch, and J. P. Toennies, Surf. Sci. 211/212, 21(1989); C.-H. Hsu, B. E. Larson, M. El-Batanouny, and C. R. Willis, Phys. Rev. Lett. 66, 3164(1991). 
III. Nonlinear And Linear Optical Diffraction Methods For Surface Diffusion Study

\section{A. General Principles of Surface Diffusion Measurement With Optical Diffraction Methods}

As mentioned in chapter II, there exist several techniques for surface diffusion measurement. The techniques that measure the tracer diffusion coefficient rely on the random walk model to obtain the quantity $D^{*}$

$$
D^{*}=\lim _{t \rightarrow \infty} \frac{\left\langle\Delta R^{2}(t)>\right.}{4 t}
$$

For those techniques that measure the chemical diffusion coefficient $D$, the fundamental equation is Fick's second law (except the Fluctuation Correlation FEM method):

$$
\frac{\partial C}{\partial t}=\nabla \cdot(D \cdot \nabla C)
$$

To ultilize this equation, two questions immediately arise: First, there must be an adsorbate density gradient in space; how is this density gradient created? Second, the time evolution of the density distribution must be known in order to extract the quantity $D$; how is this time evolution of the density distribution measured? 
In the techniques that I am going to describe for surface diffusion meas, urements, the gradient of adsorbate surface density (coverage) is generated by laser induced thermal desorption. By using a one-dimensional spatially modulated laser intensity, the desorption yields a one-dimensional monolayer grating. To probe surface diffusion of adsorbates in such a case, one monitors optical diffraction from the grating. Before diffusion, there is a finite diffraction signal from the grating. As diffusion proceeds, the adsorbate grating gets smeared out. This results in a decay of the diffraction signal, from which one can deduce the surface diffusion coefficient.

\section{B. Methods To Determine Laser-Induced-Thermal-Desorption Yield}

The key step in preparing for a diffusion measurement is to create a monolayer grating that will yield a strong enough diffraction signal. This can be achieved by laser-induced thermal desorption (LITD) with a spatial intensity modulation formed by interfering two laser beams. In order to create a prescribed adsorbate grating profile, the desorption yield versus desorbing laser energy has to be known. In this section I will describe the methods we use to measure this relation.

\section{a) Reflection Second Harmonic Generation (SHG) Method}

Reflected second harmonic generation (SHG) can be used to probe laser desorption. Because of the surface specificity, SHG is sensitive to the presence of adsorbates on a surface ${ }^{1}$. The SHG signal usually has a one-to-one 
correspondence with the adsorbate coverage and thus can be used to measure of the adsorbate coverage. In light of this, we can first measure the reflected SHG as a function of gas exposure (pressure $x$ time) to the surface. The absolute adsorbate coverage from exposure can be calibrated by from thermal desorption spectroscopy (TDS) ${ }^{2}$. Combining the results of SHG and TDS, the relation between reflected SHG and adsorbate coverage can be determined. To eliminate coniribution from the bare surface, a nonlinear interference method can be used to directly measure the adsorbate induced SHG change, namely $\left|\Delta \chi_{\text {eff }}^{(2)}(\theta)\right|^{2}$, as a function of coverage $\theta$. The details of this method can be found in Ref. [3]. A p-in(fundamental)/p-out(SH) polarization geometry is usually chosen for SHG measurements since this SHG signal is normally the strongest among all the different polarization combinations, which is due to the fact that $\chi_{z z z}^{(2)}(\theta)$ is the dominating component in the nonlinear susceptibility tensor.

If an intense enough laser pulse is applied to the adsorbate-covered sample surface, it can thermally desorption of the adsorbed atoms or molecules. The number of adsorbates that remain on the surface can then be measured by reflection SHG. Consequently, the desorption yield can be determined. By varying the energy of the laser pulse the relation of the desorption yield versus desorbing laser energy can be determined.

The set-up for the LITD experiment is shown in Fig.1, where the desorbing laser beam is aligned collinearly with the probing laser (for SHG). The probing beam radius is one tenth that of the desorbing beam and probes only the central uniform part of the desorbed area. As an example, the measured result of $\left|\Delta \chi_{\mathrm{eff}}^{(2)}(\theta)\right|^{2}$ is shown in Fig.2 for $\mathrm{CO} / \mathrm{Ni}(110)$. The CO 
desorption yield from $\mathrm{Ni}(110)$ as a function of laser energy is depicted in Fig.3(a) ${ }^{4}$.

\section{b) Linear Reflectance Method}

A second method for measuring the desorption yield of laser induced thermal desorption is linear optical reflectance. As we showed in Ref. [5] the linear reflectance can be used to monitor the adsorption and desorption processes in situ.

The experimental setup is shown in Fig.4. Since the adsorbate-induced signal is low, a lock-in technique is used. A chopped He-Ne beam first passes through a polarizer and then a Babinet phase compensator. The polarizer is adjusted to transmit equal intensities from the bare surface reflected $p$ - and $s$ polarized light. The phase compensator is adjusted to compensate the phase shift introduced by the metal surface between the $p$ and $s$ components. In this way, an analyzer set at $45^{\circ}$ with respect to the plane of incidence can cross out reflected light from the bare metal surface. Because of scattering from various optical components, this polarization cross-out can be achieved only to $10^{-5}$ of the total reflected light intensity from the surface. A lock-in amplifier can detect this background with a noise level of $1 \%$. Therefore, a change of $10^{-7}$ or larger in reflection due to adsorption of atoms or molecules on the metal surface can be detected.

With the above set-up, the following quantity

$$
S(\theta) \propto\left|r_{p}(\theta) E_{p}-r_{s}(\theta) E_{s} e^{i \Delta \phi}\right|^{2}+\Delta^{\prime}
$$




$$
\begin{aligned}
& \propto\left|\frac{r_{p}(\theta)}{r_{p}(0)}-\frac{r_{s}(\theta)}{r_{s}(0)}\right|^{2}+\Delta \\
& \propto|\Delta r(\theta)|^{2}+\Delta,
\end{aligned}
$$

is measured with $\Delta$ being the scattered background, and $r_{p}(\theta)\left(r_{s}(\theta)\right)$ and $E_{p}$ $\left(E_{S}\right)$ the field reflectivity at coverage $\theta$ and the field amplitude for the $p$ (s) polarization, respectively. The quantity $\Delta \phi=\phi_{p}-\phi_{S}$ is the phase shift between the $p$ and $s$ polarizations introduced by the Babinet phase compensator. The relation $\frac{E_{s}}{E_{p}} e^{i \Delta \phi}=\frac{r_{p}(0)}{r_{s}(0)}$ set in the experiment has been used to obtain the second equation. The last equation defines $\Delta r(\theta)=\frac{r_{p}(\theta)}{r_{p}(0)}-\frac{r_{s}(\theta)}{r_{s}(0)}$.

The principle underlying this method is simple. It is well known that atoms or molecules adsorbed on a metal surface respond to p-polarized light much more strongly than to s-polarized light. For s-polarized light, the reflectance from metal surface is almost unity and the phases between the incident and the reflected light are opposite, so that the field immediately outside the metal is nearly zero. With a molecule adsorbed at such positions, the response must be very weak. For p-polarized light, the situation is completely different. The boundary conditions at the metal surface do not require the field at the surface to be zero. This component induces a polarization on the adsorbed molecules and causes $r_{p}(\theta)$ to differ appreciably from $r_{p}(0)$. Therefore, the quantity $\Delta r(\theta)$ is finite. 
Although the principle is described for a metal substrate, it is valid for any material with a high index of refraction such as semiconductors.

In practice, the condition $\frac{E_{s}}{E_{p}} e^{i \Delta \phi}=\frac{r_{p}(0)}{r_{s}(0)}$ can never be met exactly due to mis-adjustment. If we introduce two parameters, $\delta_{\mathrm{am}}$ and $\delta_{\mathrm{ph}}$, to represent the mis-adjustment for the amplitude and the phase, respectively:

$\frac{E_{s}}{E_{p}} e^{i \Delta \phi}=\frac{r_{p}(0)}{r_{s}(0)}\left(1+\delta_{a m}\right) e^{i} \delta_{p h}$, then the final form of the measured signal is:

$$
\begin{aligned}
S(\theta) & \propto\left\{\mid \frac{r_{p}(\theta)}{r_{p}(0)}-\frac{r_{s}(\theta)}{r_{s}(0)}\left(1+\delta_{a m}\right) e^{\left.\left.i \delta_{p h}\right|^{2}\right\}+\Delta}\right. \\
& \propto\left\{\left|\Delta r(\theta)-\delta_{a m}-i \delta_{p h}\right|^{2}\right\}+\Delta .
\end{aligned}
$$

Unlike the background $\Delta$, which is incoherent with respect to $\Delta r(\theta)$, the misadjustment terms $\delta_{\mathrm{am}}$ and $\delta_{\mathrm{ph}}$ are coherent with respect to $\Delta r(\theta)$. They can be exploited in a heterodyne technique to improve the sensitivity of measuring $\Delta r(\theta)$ by about one order of magnitude ${ }^{5}$.

To reach the highest sensitivity of measuring $\Delta r(\theta)$, in principle, $\delta_{\mathrm{am}}$ and $\delta_{\mathrm{ph}}$ should be chosen as large as possible so that the signal is dominated by the interfering term $2 \operatorname{Re}\left\{\left(\delta_{a m}+i \delta_{p h}\right) \Delta r(\theta)\right\}$. Unfortunately, along with the enhancement in signal, $\delta_{\mathrm{am}}$ and $\delta_{\mathrm{ph}}$ also contribute to the noise significantly through the term $\left|\delta_{a m}-i \delta_{p h}\right|^{2}$. Therefore the compromised values of $\left|\delta_{a m}\right|^{2}$ and $\left|\delta_{\mathrm{ph}}\right|^{2}$ should be on the order of $1 \times 10^{-5}$ of the reflected light intensity. 
As mentioned earlier, the minimum reflectance change $S(\theta)$ we are able to measure is $10^{-7}$ of the reflected light intensity. Although it is two orders of magnitude better than other schemes for measuring $\Delta R(\theta) / R^{6,7}$, the sensitivity of measuring $\Delta r(\theta)$ is only comparable to those schemes, in which the effective $\delta_{\mathrm{am}}$ and $\delta_{\mathrm{ph}}$ have been chosen nearly unity and therefore the strength of the signal $2 \operatorname{Re}\left\{\left(\delta_{a m}+i \delta_{p h}\right) \Delta r(\theta)\right\}$ is correspondingly about two orders of magnitude larger. As a result, our scheme has not improved the sensitivity for $\Delta r(\theta)$. The advantage here is the simplicity of the set-up.

With this linear reflectance technique, we measured the adsorption and the laser induced thermal desorption for $\mathrm{CO} / \mathrm{Ni}(110)$. In Fig. 5 we plot the differential reflectance signal $S(\theta(t))$ as a function of time. The signal increases as we dose $\mathrm{CO}$ onto the $\mathrm{Ni}(110)$ surface until saturation, which corresponds to a full CO monolayer. At time $t \sim 350 \mathrm{sec}$ a strong laser pulse with $1.1 \mathrm{~J} / \mathrm{cm}^{2}$ is applied to desorb $\mathrm{CO}$ from the surface. Immediately the signal drops to a low level which corresponds to a low CO coverage. At time $t=410 \mathrm{sec}$ the signal recovers as more $\mathrm{CO}$ molecules are adsorbed on to the surface until saturation is reached again. A second laser puls $\Theta$ with $0.9 \mathrm{~J} / \mathrm{cm}^{2}$ is applied some time later and the signal drop is less, corresponding to a ieduceú CO desorption.

With a calibrated relation between coverage and exposure time as obtained by $\operatorname{TDS}^{2}$, the differential reflectance signal $S(\theta(t))$ can be related to CO coverage. In Fig. 6, the desorption yield is shown as a function of desorption laser energy for three different initial coverages. The accuracy in determining the cesorption yield is better than 0.05 monolayer, which is much better than reflection SHG. In principle a sensitivity of 0.01 monolayer can be achieved. We are basically limited by the long term instability from mechanical drifts of the optics and sample in our set-up. 


\section{c) Laser Heating Model and Laser Induced Thermal Desorption}

The reason that a laser pulse can desorb adsorbed atoms or molecules from the surface is that the laser heats up the surface in a very short time. Models have been developed for this process ${ }^{8}$. In all the models, the absorbed laser energy is instantaneously converted into heat, which results in an increase in the surface temperature. The equations that govern this temperature change are the heat-conduction equation and the Fouric r's law:

$$
\begin{aligned}
& \nabla . J(r, t)+\rho C_{p} \frac{\partial T(r, t)}{\partial t}=A(r, t), \\
& J(r, t)=-K \nabla T(r, t),
\end{aligned}
$$

where $p, C_{p}, K$ are the density, heat capacity and heat conductance of the substrate, respectively and $\mathbf{J}$ is the heat flux. The laser heating effect is represented by the source term $A(r, t)$, or by boundary conditions, depending on the model. The two equations above can be combined into a diffusion equation

$$
\nabla^{2} T(r, t)-\frac{1}{K} \frac{\partial T(r, t)}{\partial t}=-\frac{A(r, t)}{K},
$$

with $k=K / p C_{p}$, the heat diffusion constant. In our situation the laser spot is usually very large compared with the heat diffusion length $\xi \sim\left(\kappa \tau_{p}\right)^{1 / 2} \sim 1 \mu$. Therefore the lateral heat diffusion on the time scale of laser pulse duration $\tau_{p}$ can be neglected and the lateral temperature dependence can be treated quasi-statically. This reduces the heat diffusion equation to a one dimension. 
There are two common ways to treat the laser heating effect: one is called surface heating, the other, volume heating. In the surface heating model, the source term is assumed to be a delta function and can be effectively represented by a boundary condition

$$
\left.K \frac{d T(r, t)}{d z}\right|_{z=0}=-(1-R)|(r, t)|_{z=0}
$$

where $R$ is the reflectance, and $I(r, t)$ is the laser intensity at the surface. Using the fact that the temperature far into the bulk of the substrate is constant , the surface temperature rise is given by

$$
\Delta T(t)=\frac{\Delta E(r)}{\Delta A}(1-R) \cos \theta_{\text {inc }} \frac{1}{\sqrt{\pi} \tau_{p}} \int_{-\infty}^{t} \frac{d t^{\prime}}{\sqrt{\pi \rho C_{p} K}} \frac{\exp \left(-t^{\prime 2} / \tau_{p}^{2}\right)}{\sqrt{t-t^{\prime}}}
$$

where $\triangle E / \triangle A$ is the laser energy impinging on the surface, and $\theta_{\text {inc }}$ is the incident angle. For the surface heating model to be valid, the heat diffusion length $\xi$ must be much longer than the laser absorption depth. This condition can be met by metals, which have a very high thermal conductivity and a large absorption coefficient for light. However, for materials such as semiconductors, which have reasonable thermal conductivity but a low light absorption coefficient, the surface heating model may fail. A better way to treat the problem is the volume heating model, which takes

$$
\begin{aligned}
& A(r, t)=I_{m}(1-R) \alpha \exp (-\alpha z) f(r) q(t) \quad \text { and } \\
& \left.\frac{d T(r, t)}{d z}\right|_{z=0}=0
\end{aligned}
$$


where functions $f(r), q(t)$ describe the spatial and temporal shape of the laser pulse in the medium, $I_{m}$ is the peak intensity at the surface, and $\alpha$ the absorption coefficient. The solution for the surface temperature rise is now given by

$$
\Delta T(t)=\frac{I_{m}^{(1-R) \alpha K}}{2 \sqrt{\pi} K} f(r) \int_{-\infty}^{t} d t^{\prime} \exp \left(-t^{\prime} 2 / \tau_{p}^{2}\right) \exp \left[\kappa \alpha^{2}\left(t-t^{\prime}\right)\right] \operatorname{erfc}\left[\alpha\left[\kappa\left(t-t^{\prime}\right)\right]^{1 / 2}\right]
$$

where the complementary error function is defined by

$$
\operatorname{erfc}(t)=\frac{2}{\sqrt{\pi}} \int_{t}^{\infty} \exp \left(-x^{2}\right) d x
$$

In the limit $\alpha->\infty$, the result of volume heating model reduces to that of the surface heating model.

For the laser induced thermal desorption experiments on $\mathrm{CO} / \mathrm{Ni}(110)$, the reflectance of $\mathrm{Ni}$ at the incident angle $\theta_{\text {inc }} \sim 0^{\circ}$ is $\mathrm{R}=0.728$, the laser pulse width is $\tau_{p}=10$ nsec, and the density, heat capacity and heat conductance of Ni are $\rho=8.902 \mathrm{~g} / \mathrm{cm}^{3}, C_{p}=6.23 \mathrm{cal} / \mathrm{mol} . \mathrm{K}$ and $K=91 \mathrm{~W} / \mathrm{m} . \mathrm{K}$, respectively ${ }^{9}$. With the desorption rate of $\mathrm{CO}$ from $\mathrm{Ni}(110)$ given by a first order process

$$
\frac{d \theta}{d t}=-v \theta e^{-E_{d e s} k_{B} T(t)}
$$

$\checkmark$ the desorption pre-exponential factor, $E_{\text {des }}$ the desorption energy, and $k_{B}$ the Boltzmann constant, the thermal desorption yield is 


$$
\Delta \theta(t)=\theta_{s}\left\{1-\exp \left(-\int_{-\infty}^{t} v e^{-E_{d^{\prime}} / k_{B} T\left(t^{\prime}\right)} d t^{\prime}\right)\right\} .
$$

In Fig. 7, we have shown the laser-induced surface temperature rise $\Delta T(t)$ and the desorption yield $\Delta \theta(t)$ from such a calculation. The desorption yield data from both methods now can be fit by the surface heating model. The solid lines in Fig.3(a) and Fig. 6 are fits of the data using Eqs. (9) and (13). The fitting parameters for the desorption data with reflection SHG method are $v=1 \times 10^{14}$ and $E_{d e s}=28 \mathrm{kcal} / \mathrm{mol}$. For the data with the linear reflectance method, $v=$ $1 \times 10^{14}$ and $E_{\text {des }}=30 \mathrm{kcal} / \mathrm{mol}$ for initial coverage $\theta_{0}=1.0$ and $v=2 \times 10^{14}$ and $E_{\text {des }}=32 \mathrm{kcal} / \mathrm{mol}$ for $\theta_{0}=0.50$ and $\theta_{0}=0.25$. These results are in good agreement with previous measurements by other techniques ${ }^{10}$.

\section{Adsorbate gratings}

As pointed out in the introduction, the creation of a monolayer grating is crucial for diffusion measurements with optical diffraction methods. To make a monolayer adsorbate grating, we interfered two laser beams at the sample surface to produce a spatially modulated light intensity pattern

$I(x)=I_{0}\left[1+r \cos \left(\frac{2 \pi x}{s}\right)\right]$,

where $I_{0}$ is the average intensity, $s$ the grating spacing, $r$ the contrast of the interference pattern, and $x$ the coordinate on the surface. With this intensity profile and the relation of desorption yield versus laser intensity from the 
measurements described in section 2, the adsorbate yrating, namely the coverage as a function of $x$ can be mapped out. This is shown in Fig. 3.

The diffraction signal from this adsorbate grating can be estimated. In the SH diffraction case, the adsorbate induced second order nonlinear susceptibility $\Delta \chi_{\text {eff }}^{(2)}(\theta)$ is modulated by the adsorbate grating $\theta(x)$ with a periodicity s, therefore the diffraction signal of the $n$-th order is given by

$$
\begin{aligned}
& S_{n} \sim\left|A_{n}\right|^{2}, \\
& A_{n}=\lim _{L \rightarrow \infty} \stackrel{2}{L} \int_{-L / 2}^{L / 2} \chi_{\text {eff }}^{(2)}(\theta(x)) \exp \left(i \frac{2 n \pi x}{s}\right) d x,
\end{aligned}
$$

where $L$ is the dinension of the grating. Assuming a simple case with $\chi_{e f f}^{(2)}(\theta(x))$ $=A+B \theta$. The ratio

$$
\frac{S_{n}}{S_{0}} \sim \frac{1}{n^{2} \pi^{2}}\left|\frac{B \theta_{n}}{A+B \theta_{0}}\right|^{2}
$$

can be estimated with known $A, B, \theta_{0}$ and $\theta_{n}$, where $\theta_{n}$ is the $n$-th Fourier component of the adsorbate grating. Since $S_{0}$ can be easily measured for any system without a grating on the surface, the diffraction signals can then be estimated by Eq. (16).

In the linear diffraction case, the estimate is very similar. We only have to replace $\chi_{\text {eff }}^{(2)}(\theta)$ by the field reflectivity $r(\theta)$. That is

$$
\frac{s_{n}}{s_{0}} \sim\left|\frac{r_{n}}{r_{0}}\right|^{2}
$$




$$
\sim \frac{1}{n^{2} \pi^{2}}\left|\frac{r(\theta)-r(0)}{r(0)}\right|^{2}
$$

with $r_{n}$ defined as

$$
\left.r_{n}=\lim _{L \rightarrow \infty} \frac{2}{L} \int_{-L / 2}^{L / 2} r(\theta(x, t)) \exp \left(\frac{2 n \pi x}{s}\right)\right) d x, \quad n=0,1,2 \ldots
$$

If the absolute change in the reflectivity is known, the absolute strength of the diffraction signal can be estimated. Typically, $r(\theta)-r(0)$ is about $10^{-4}-10^{-3}$ so the first order diffraction signal will be $10^{-9}-10^{-7}$ of the reflection.

The periodicity $s$ of the grating is determined by the angle between the two interfering laser beams. With the half angle denoted by $\phi$, the grating spacing is given by

$$
s=\frac{\lambda}{2 \sin \phi} .
$$

The choice of this spacing depends on the diffusion rate. As we will see in the next section, the diffusion coefficient $D$ is related to the decay time constant $\tau$ of the nth-order diffraction by $\tau=s^{2} / 8 \pi^{2} n^{2} D$. The maximum decay time constant we can measure is limited by the long term stability of the system and the rate of contamination of the sample surface. This time is typically one hour. The minimum decay time constant is limited by the data acquisition time. In the SH diffraction case it is about half an hour, but in the linear diffraction case it can be less than a second. It is then seen that properiy adjusting the grating spacing, the dynamic range of the diffusion coefficient we can measure can extend from $10^{-6}$ to $10^{-15} \mathrm{~cm}^{2} / \mathrm{sec}$. 
The depth of the adsorbate grating should be chosen according to the signal-to-noise ratio of the diffraction signal and can be controled by the intensities of the two interfering laser beams. Typically, an initial signal-to-noise ratio of 10 is required for diffusion measurement with $20 \%$ accuracy in determining the diffusion coefficient. For large response systems the adsorbate grating can be made shallow, i.e., with a small coverage modulation. For small response systems the adsorbate grating should have a large coverage modulation. A control of this coverage modulation relies on the control of the two laser beams' intensities. As we will see, the linear diffraction method has the capability for detecting shallow gratings, while the $\mathrm{SH}$ diffraction method may require a deep grating.

\section{Optical Diffraction as a Probe of Surface Diffusion}

\section{a) Second Harmonic Diffraction Probe}

In the second harmonic diffraction case, the surface specificity of the signal eliminates the background from the bulk metal so that no modulation scheme is necessary. This can be seen as follows. Since the scattered light intensity in the diffraction direction is roughly $10^{-6}$ of the reflected intensity and the diffraction signal is on the order of $1 / 10$ of the adsorbate-induced $\mathrm{SH}$ reflection signal, the signal-to-background ratio is then $10^{5}$ due to the fact that the change in SHG signal due to adsorption is comparable to the bare surface signal. The optical arrangement for the diffusion experiment is shown in Fig.8. A single-mode Q-switched Nd:YAG laser with a pulse width of 10ns at $1.06 \mu \mathrm{m}$ was used for both the creation of the adsorbate grating and the SH diffraction 
measurement. The detection of the diffraction signal relies on the knowledge of the diffraction spot position. One can calculate this diffraction spot position from the relation

$$
k_{x n}(2 \omega)=2 k_{x}(\omega)+\frac{2 \pi n}{s}
$$

where $k_{x n}(2 \omega)$ and $k_{x}(\omega)$ are the tangential components of the wavevectors for the $\mathrm{n}$-th order diffraction second harmonic beam and the incident fundamental beam respectively. In practice, the alignment of the detection system then is performed using the calculated angle between the reflection direction and the diffraction direction.

The diffusion of atoms or molecules on a surface can be related to the diffraction signal in a simple way. The solution of the one dimensional diffusion equation

$$
\frac{\partial \theta}{\partial t}=\frac{\partial}{\partial x}\left(D \frac{\partial \theta}{\partial x}\right)
$$

with a periodic initial condition can be expressed in terms of a Fourier series expansion:

$$
\theta(x, t)=\theta_{0}+\sum_{n=1}^{\infty} \theta_{n}(t) \cos (2 n \pi x / s)
$$

If $D$ is assumed to be independent of the coverage $\theta$, we further obtain 


$$
\theta(x, t)=\theta_{0}+\sum_{n=1}^{\infty} \theta_{n}^{0} \cos (2 n \pi x / s) \exp \left(-4 n^{2} \pi^{2} D t / s^{2}\right)
$$

From Eq. (15) we find the $n$-th order diffraction signal to be

$$
\begin{aligned}
S_{n}(t) \propto\left|\theta_{n}\right|^{2} & \\
& =S_{O n} \exp \left(-8 \pi^{2} n^{2} D t / s^{2}\right) .
\end{aligned}
$$

with the diffusion coefficient only related to the decay time $\tau=s^{2} / 8 \pi^{2} n^{2} D$ but not to the signal strength if the optical response $\chi_{\text {eff }}^{(2)}(\theta(x))$ is linear with the coverage $\theta$. With this important result the diffusion coefficient measurement can be achieved simply by measuring the diffraction signal decay.

\section{b) Linear Diffraction Probe}

The disadvantage of $\mathrm{SH}$ diffraction is that the nonlinear optical process is usually very weak so the signal strength can be very small. However, linear optical processes can have tremendously stronger response than SHG. The difficulty in applying linear diffraction for probing surface diffusion is that the signal from a monolayer grating of adsorbates is buried in a much stronger scattered background, which is typically $10^{-6}$ of the reflected intensity. This renders a direct measurement of diffraction signal difficult.

To overcome this difficulty, we have to use a method in which the diffracted signal is modulated differently from the scattered background. One way to achieve this is polarization modulation. If we realize that the scattered background light is arising from the roughness of the surface and the defects in 
the bulk, then the intensity is not strongly dependent on the incident light polarization. In contrast, the diffraction signal from a monolayer grating is a response to the electric field at the surface and therefore is strongly polarization dependent. For s-polarization the electric field at the surface is almost zero, for $\mathrm{p}$-polarization the electric field at the surface is nonzero. Therefore, modulating the polarization of the incident light suppresses the scattered background. The suppress can be achieved to as much as a factor of $10^{5}$. With a signal strength comparable with the background the signal-to-background ratio is about $10^{5}$. In this way, the sensitivity of detecting a monolayer grating with optical linear diffraction is greatly enhanced compared to the $\mathrm{SH}$ diffraction case.

The linear diffraction set-up is shown in Fig. 9. A polarized He-Ne beam $(5 \mathrm{~mW})$ first passes through a photoelastic modulator which modulates the polarization sinusuodally at $50 \mathrm{kHz}$. Then the beam is enlarged by about a factor of 3 with a telescope. This beam is slightly focused by a $2 \mathrm{~m}$ lens onto the sample surface. A PMT detector is aligned in the first order diffraction direction by using the relation

$$
s\left(\sin \theta_{n}-\sin \theta_{0}\right)=n \lambda,
$$

where $\theta_{n}$ is the $n$-th order diffraction angle, $\theta_{0}$ is the incident angle, and $n= \pm 1$ for the first order diffraction. Experimentally, this direction can also be determined if a permanent grating is created with the two interfering beams. The diffusion coefficient is deduced in the same way as in the SH diffraction case. The diffraction signal detected by the set-up in Fig. 8 is expressed as

$$
S_{n}=\left|r_{n p}\right|^{2} I_{p}-\left|r_{n s}\right|^{2} I_{s}
$$


with $\left.\quad r_{n K}=\lim _{L \rightarrow \infty} \frac{2}{L} \int_{-L / 2}^{L / 2} r_{\kappa}(\theta(x)) \exp \left(i \frac{2 n \pi x}{s}\right)\right) d x$, for $\kappa=s, p$.

With the incident light being polarization modulated to give intensities

$$
\begin{aligned}
& I_{p} \sim\left|E_{p}\right|^{2} \cos ^{2}\left(\psi \sin \omega t+\phi_{0}\right), \\
& I_{s} \sim\left|E_{s}\right|^{2} \sin ^{2}\left(\psi \sin \omega t+\phi_{0}\right),
\end{aligned}
$$

with $\psi$ the phase modulation amplitude, $\omega$ the modulation frequency, and $\phi_{0}$ the residual phase in the modulator, the lock-in amplifier then detects a signal of

$$
s_{n}=\frac{V_{p}+V_{s}}{2}+\frac{V_{p}-V_{s}}{2} \cos \left(\psi \sin \omega t+\phi_{0}\right)
$$

with $V_{p}$ and $V_{s}$ the diffraction signal strength for $p$ and $s$ polarizations respectively. The cosine function in Eq. (27) can be expanded into Fourier series and the coefficients of the Fourier series are given by different orders of Bessel functions. With $\phi_{0}=0$, only even harmonics have nonvanishing coefficient and with $\phi_{0}=\pi / 2$ only odd harmonics have nonvanishing coefficient. If we desire to measure a signal with the fundamental frequency, the phase $\phi_{0}$ $=\pi / 2$ has to be introduced with a quarter wave plate.

The optical diffraction methods off an adsorbate grating for surface diffusion measurement have a number of very attractive features. First, it involves a simple one-dimensional diffusion process for which the data analysis is relatively straightforward. Second, by properly orienting the grating, the 
diffusion coefficient along any direction on the surface can be directly measured. Third, as an optical method, the technique can be applied to a wide variety of adsorbate-substrate systems. Fourth, with a tunable probe beam selectively probing particular species of adsorbates, surface diffusion of individual components of a mixed adsorbate layer can be monitored. This allows the study of influence of surface diffusion on surface reactions. Finally, the technique can be used to study other forms of surface diffusion such as diffusion of electronic or vibrational excitations.

\section{E. Measuring Coverage Dependence of Diffusion}

In order to study the coverage dependence of diffusion, two schemes are considered. The first scheme is simple and easily implemented. The second one involves some careful considerations and at present still is difficult to implement.

In the first scheme, we make an adsorbate grating with shallow depth $\Delta \theta$. Upon establishing a uniform coverage of adsorbates through dosing, we use two laser beams with predescribed intensities to interfere at the surface. The intensities of the two beams are chosen so that only a little desorption takes place to create an adsorbate grating with a small modulation depth $\Delta \theta$. The choice of this depth is limited by the strength of the diffraction signal for the specific system under study. In the $\mathrm{CO} / \mathrm{Ni}(110)$ case, the depth is chosen to be

$0.05 \mathrm{ML}$. With such a shallow grating the diffusion coefficient can be very well approximated as a constant and can be described by Eq. (23). With varying 
initial coverages the dependence of the diffusion coefficient with coverage can be determined.

The second scheme for coverage dependent diffusion study is principally the same as the Boltzmann-Matano method ${ }^{12}$, where a step coverage was initially created. With laser desorption by a spatially modulated intensity, a single step coverage profile is difficult to achieve and even if it is achieved, the diffraction signal would be very weak. Naturally, a series of step-like coverage profiles with a periodicity defined by the interfering beams can be easily realized by choosing a large enough intensity modulation. If we can detect not only the first order diffraction signal but also all the higher orders, in principle, the evolution of the coverage profile can be determined. With this known coverage profile as a function of time, it is possible for us to solve the diffusion equation numerically and find the coverage dependent diffusion coefficient.

To examine this clearly, let us start with the Fourier expansions of the coverage and the diffusion coefficient

$$
\begin{aligned}
& \theta(x, t)=\theta_{0}+\sum_{n=1}^{\infty} \theta_{n}(t) \cos (2 n \pi x / s) \\
& D(\theta(x, t))=D_{0}+\sum_{n=1}^{\infty} D_{n}(t) \cos (2 n \pi x / s) .
\end{aligned}
$$

Assuming the Fourier components as functions of time in the expansion of coverage are known from the multiple diffraction measurements, then substituting them into the diffusion equation, 


$$
\frac{\partial \theta}{\partial t}=\frac{\partial}{\partial x}\left(D \frac{\partial \theta}{\partial x}\right)
$$

we obtain

$$
\begin{aligned}
& \sum_{n=0}^{\infty} \frac{\partial \theta_{n}(t)}{\partial t} \cos (2 n \pi x / s) \\
& =\frac{\partial}{\partial x}\left(\sum_{n=0}^{\infty} D_{n}(t) \cos (2 n \pi x / s) \frac{\partial}{\partial x} \sum_{n=0}^{\infty} \theta_{n}(t) \cos (2 n \pi x / s)\right) .
\end{aligned}
$$

By carrying out the derivatives and collecting the terms with the same base function, we can show that

$$
\begin{gathered}
\frac{\partial \theta_{0}(t)}{\partial t}=0, \\
\frac{\partial \theta_{1}(t)}{\partial t}=-\frac{\pi^{2}}{a^{2}}\left(D_{0}(t)-\frac{1}{2} D_{2}(t)+\frac{\sum_{n \neq 1} n\left[D_{n+1}(t) \theta_{n}(t)-D_{n-1}(t) \theta_{n}(t)\right]}{2 \theta_{1}(t)}\right) \theta_{1}(t),
\end{gathered}
$$

and generally,

$$
\frac{\partial \theta_{m}(t)}{\partial t}=-\frac{m^{2} \pi^{2}}{a^{2}}\left(D_{0}(t)-\frac{1}{2} D_{m+1}(t)+\frac{\sum_{n \neq m} n\left[D_{n+1}(t) \theta_{n}(t)-D_{n-1}(t) \theta_{n}(t)\right]}{2 \theta_{m}(t)}\right) \theta_{m}(t) .
$$

These are coupled equations for the $D_{n}(t)$ 's. By solving them we should be able to obtain all the components of $D_{n}$. From them we can easily construct 


$$
D(\theta(x, t))=D_{0}+\sum_{n=1}^{\infty} D_{n}(t) \cos (2 n \pi x / s)
$$

There are two ways to determine $D$ as a function of $\theta$ from the above equation. The first one is to sit at a fixed position $x$ and watch the coverage change as a function of time. For any time $t$, there is a corresponding $\theta$ and a corresponding $D(\theta)$. A complete mapping for $D$ from variable $t$ to $\theta$ can be achieved if the change of coverage at the chosen position $x$ covers the full range. The second way is to fix the time and examine the coverages at different positions within a period of the grating. $D(\theta)$ can then be mapped out through the variable $x$.

In order to complete the discussion, we still have to relate the Fourier components of the coverage to all different orders of diffraction signals. For such a purpose we need to know the coverage dependent reflectivity $r(\theta)$ first. This can be approximately measured by the method we discussed in section 2 . For an adsorbate grating given by Eq. (25) we have

$$
r(\theta(x, t))=r_{0}+\sum_{n=1}^{\infty} r_{n}(t) \cos (2 n \pi x / s)
$$

and the $n$-th order diffraction signal is directly proportional to the $\left|r_{n}(t)\right|^{2}$. To relate these measured quantities to the Fourier components of coverage $\theta_{n}(t)$, we ultilize the inverse function $\theta(r)$ of $r(\theta)$ and then solve them in terms of $r_{n}(t)$.

To elaborate on this point further, let us expand $r(\theta)$ into a power series,

$$
r(\theta)=\sum_{n=0}^{\infty} a_{n}\left(\theta-\theta_{0}\right)^{n}
$$


around the average coverage $\theta_{0}$. Substituting the Fourier expansions for both the coverage and the reflectivity into the above equation, we can then relate the measured quantities $r_{n}(t)$ to $\theta_{n}(t)$. One important point is that the phases of the Fourier components of the reflectivity are not directly measurable since only the diffraction intensity was measured and not the diffraction field. In simple cases such as a real functional dependence, the phases reduce to plus or minus and it is possible to determine them by constructing a self consistent coverage evolution profile. In more complicated situations direct determination of phases is needed. This is yet to be solved in this multiple diffraction scheme.

Despite the difficulties in the second scheme, it is still very intriguing. With a slightly complicated set-up to measure all the orders of diffraction , coverage dependent diffusion coefficient measurement requires the creation of only one single grating. The data analysis is then supposed to give complete information on $D(\theta)$ with $\theta$ almost as a continuous variable. This is certainly in strong contrast with the first scheme, where the coverage dependence measurement is done by varying the initial coverage. Since it can save a tremendous amount of experimental time, the second scheme is attractive. The additional problem is that if the diffusion coefficient is not only dependent on the coverage but also the coverage gradient, then the measured $D(\theta)$ in the two schemes can be different. Even though theoretically it is possible, practically, that effect may be very small as compared to the coverage dependence itself. 


\section{References:}

${ }^{1}$ For reviews of surface SHG see G. I. Richmond, J. M. Robinson, and V. L. Shannon, Prog. Surf. Sci. 28, 1(1988); T. F. Heinz and G. A. Reider, Trends Anal. Chem. 8, 235(1989); Y. R. Shen, Nature 337, 519(1989).

2 For example, R. J. Behm, G. Entl, and V. Penka, Surf. Sci. 160, 387(1985).

${ }^{3}$ X. D. Zhu, Winfried Daum, Xu-dong Xiao, R. Chin and Y. R. Shen, Phys. Rev. B 43, 11571(1991).

${ }^{4}$ Xu-dong Xiao, X. D. Zhu, Winfried Daum, and Y. R. Shen, accepted for publication by Phys. Rev. B.

${ }^{5}$ Xu-dong Xiao, Yuanlin Xie, and Y. R. Shen, to be published.

${ }^{6}$ Graciela B. Blanchet, P. J. Estrup, and P. J. Stiles, Phys. Rev. Lett. 44, 171(1980); Phys. Rev. B. 22, 3655(1981).

${ }^{7}$ D. E. Aspnes, J. Vac. Sci. Technol. B 3, 1498(1985); 1138(1985); D. E. Aspnes, J. P. Harbison, A. A. Studna and L. T. Florez, Appl. Phys. Lett. 52, 957(1988).

8 J. H. Bechtel, J. Appl. Phys. 46, 1585(1975).

9 American Institute of Physics Handbook, 3rd ed., ed. by D. Gray (McGraw-Hill, New York, 1972), pp. 4-106, 4-154.

${ }^{10}$ C. S. Feigerle, S. R. Desai, and S. H. Overbury, J. Chem. Phys. $93,787(1990)$.

${ }^{11}$ X. D. Zhu, A. Lee, and A. Wong, Appl. Phys. A. 52, 317(1991).

${ }^{12}$ For example, W. Jost, Diffusion in Solids, Liquids, Gases, (Academic Press, New York, 1960). 


\section{Figure Captions}

Figure 1: Optical set-up for experiment using optical second harmonic generation to probe laser induced thermal desorption. F1 and F2 are color filters and $Q$ is a quartz plate employed to cancel the SHG signal from the bare $\mathrm{Ni}(110)$ surface by interference.

Figure 2: $\left|\Delta \chi_{\mathrm{eff}}^{(2)}(\theta)\right|^{2}$ versus coverage $\theta$ measured by SHG with the plane of incidence parallel to $[1 \overline{1} 0]$ and the $p$-in/p-out polarization combination.

Figure 3: (a) Desorption mass yield versus desorbing laser energy as measured by laser induced thermal desorption. The solid line is a theoretical calculation from Eq. (3) with $v=1 \times 10^{14}$ and $E_{\text {des }}=28 \mathrm{kcal} / \mathrm{mol}$. The dashed line along the data points is for eyeguide; (b) Laser energy distribution at the surface from two interfering laser beams; (c) The resulting coverage grating created by the laser energy distribution in (b).

Figure 4: Sketch of the experimental set-up for differential reflectance measurement. $P$ is the polarizer, $A$ the analyzer, and $C$ the phase compensator.

Figure 5: Differential reflectance signal $S(\theta(t)$ ) versus time for $C O$ adsorption (at CO pressure $2.5 \times 10^{-8}$ torr) and laser induced thermal desorption kinetics on $\mathrm{Ni}(110)$ surface. 
Figure 6: Laser induced thermal desorption yield versus laser energy for CO/Ni(110) system at three different initial coverages: $\theta_{0}=0.25, \theta_{0}=0.5$, and $\theta_{0}=1.0$. The solid curves are fits from a simple thermal desorption model discussed in the text.

Figure 7: Surface temperature rise $\Delta T(t)$ and laser-induced desorption yield $\Delta \theta(t)$ as a function of time calculated from Eq.(9) and(13). The laser energy and the desorption parameters used for the calculation are $1.0 \mathrm{~J} / \mathrm{cm}^{2}$ and $v=1 \times 10^{14}$ and $E_{d e s}=30 \mathrm{kcal} / \mathrm{mol}$ respectively. The surface temperature levels at a different value from its initial one due to the fact that no heat dissipation mechanism has been introduced in the model.

Figure 8: Experimental set-up for surface diffusion experiment with $\mathrm{SH}$ diffraction detection. A single laser shot at $1.06 \mu \mathrm{m}$ is always used to generate an adsorbate grating. The decay of the grating is monitored by the first-order SH diffraction using the $0.532 \mu \mathrm{m}$ probe beam.

Figure 9: Schematic of the experimental arrangement for detection of first-order linear diffraction from a monolayer grating on $\mathrm{Ni}(110)$. The $\mathrm{He}-\mathrm{Ne}$ laser has been polarization-modulated by entering into the chamber. The diffraction angle with respect to reflection can be calculated. 


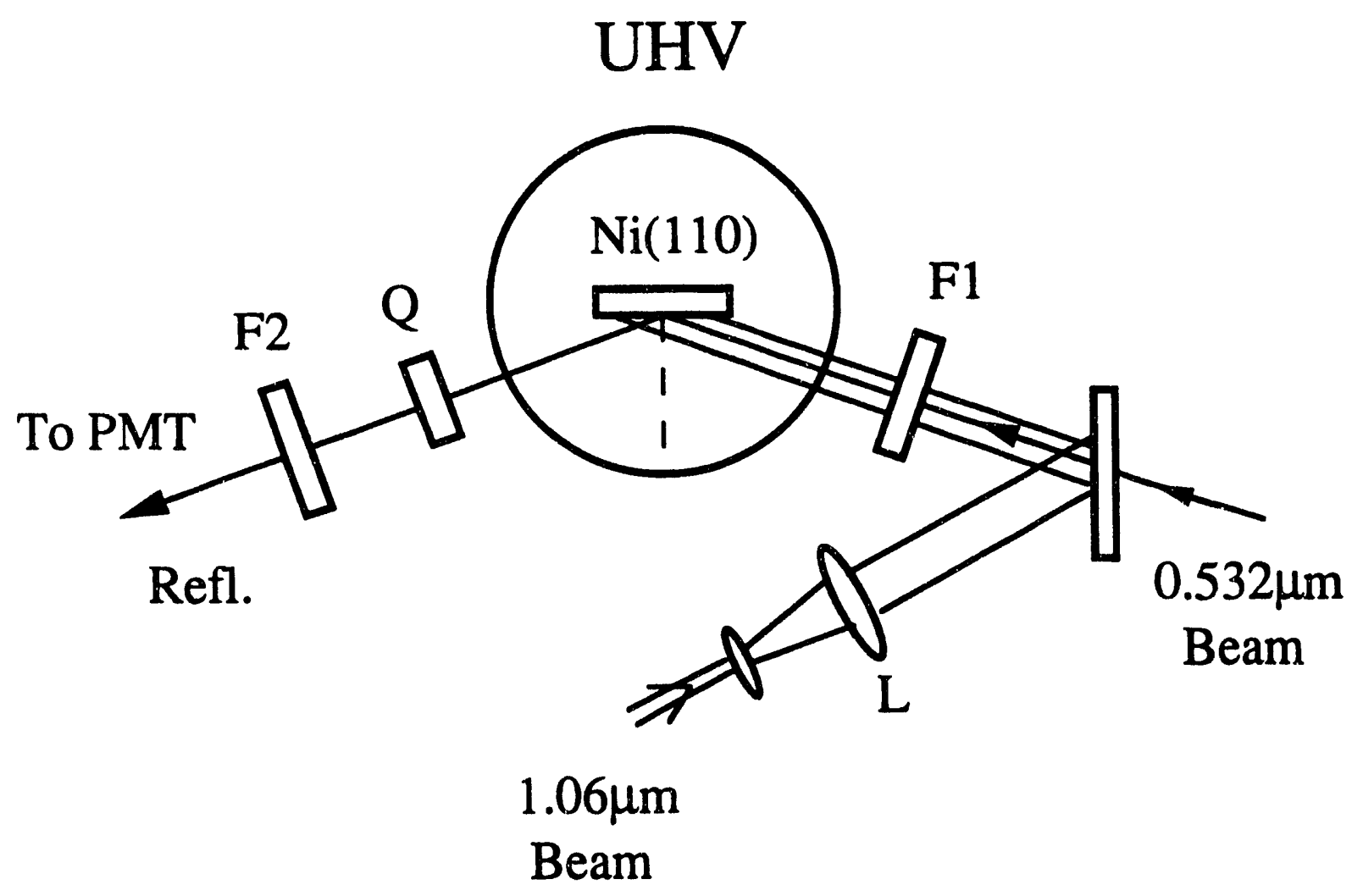

Figure 1 


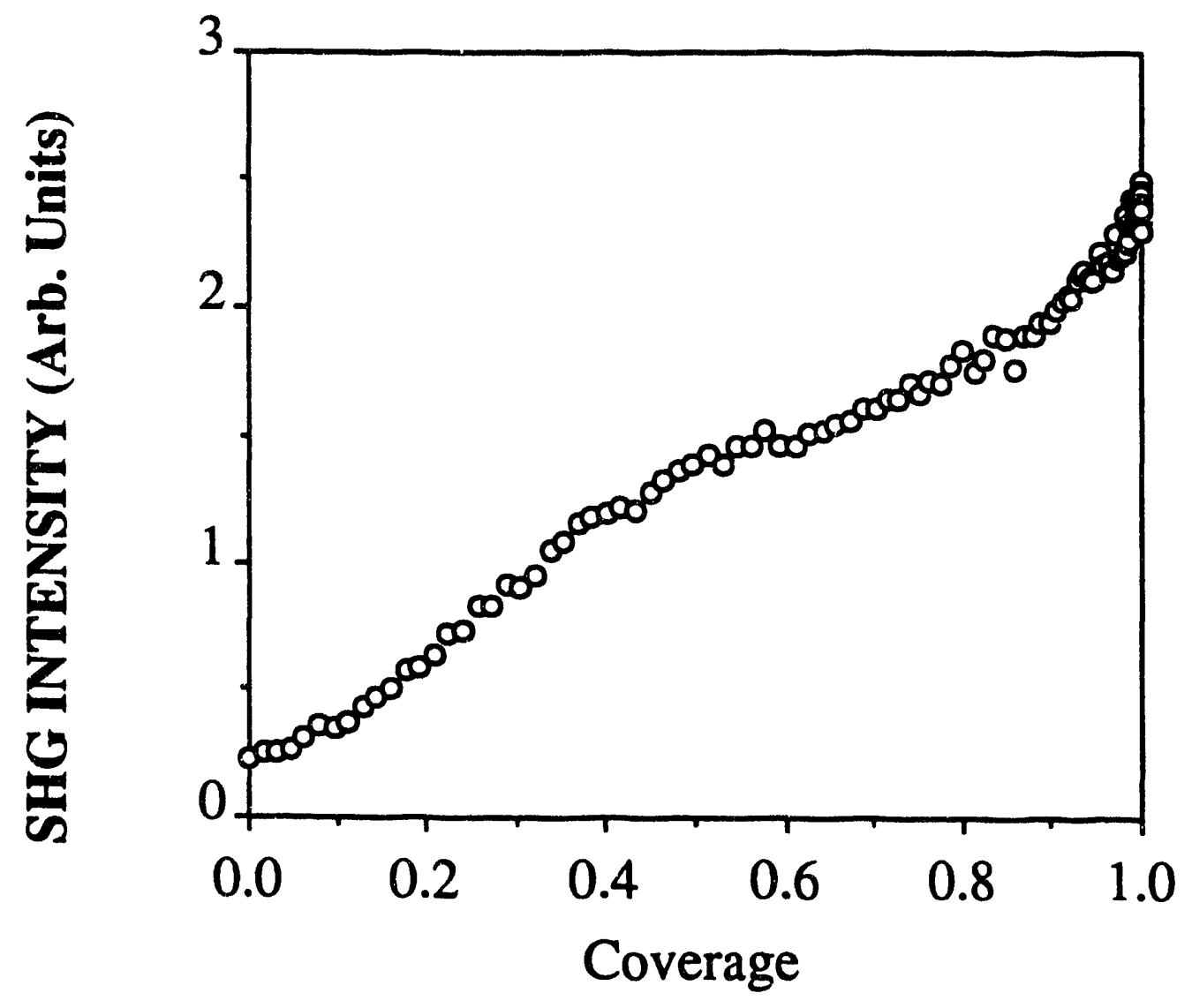

Figure 2 


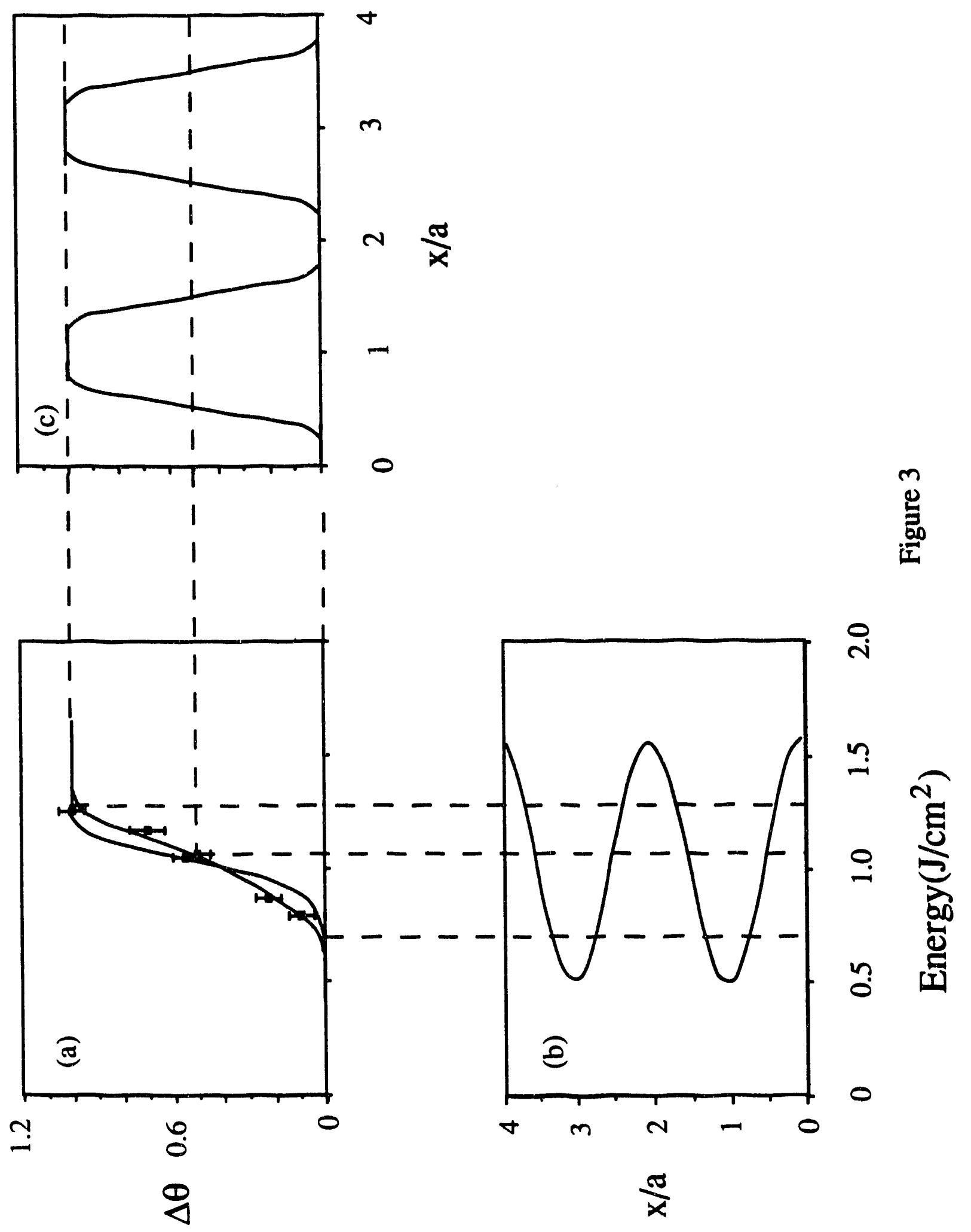




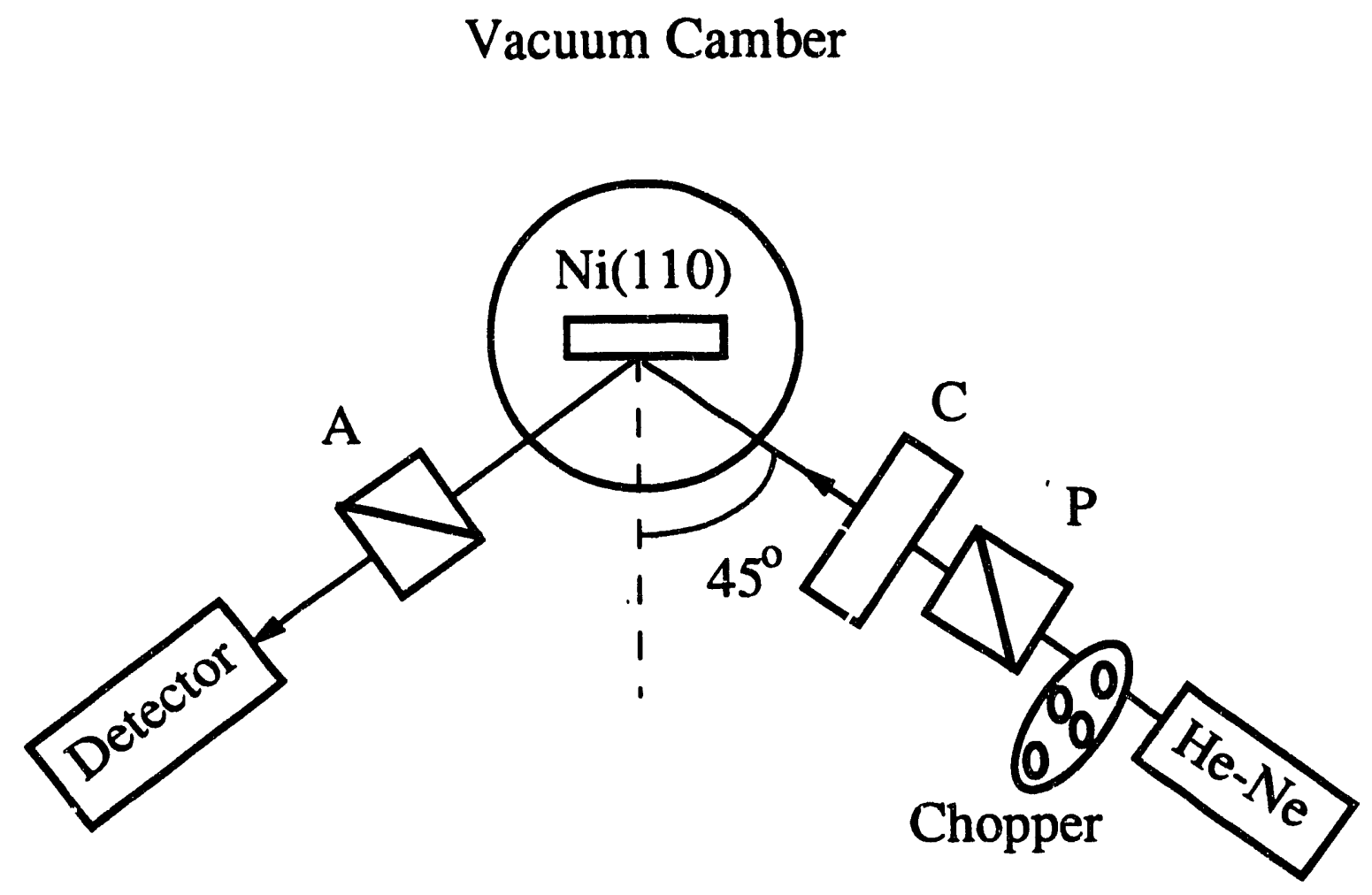

Figure 4 


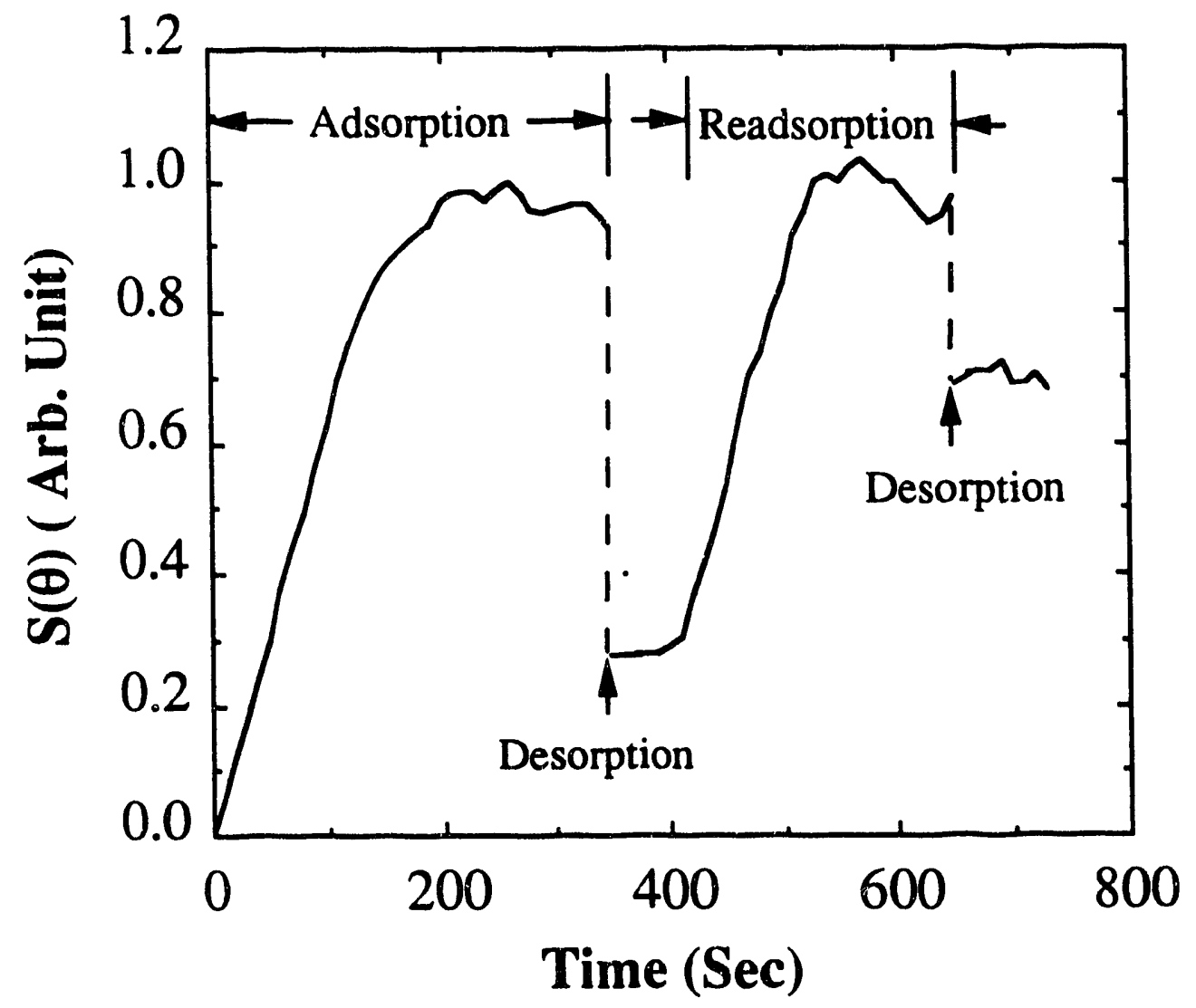

Figure 5 


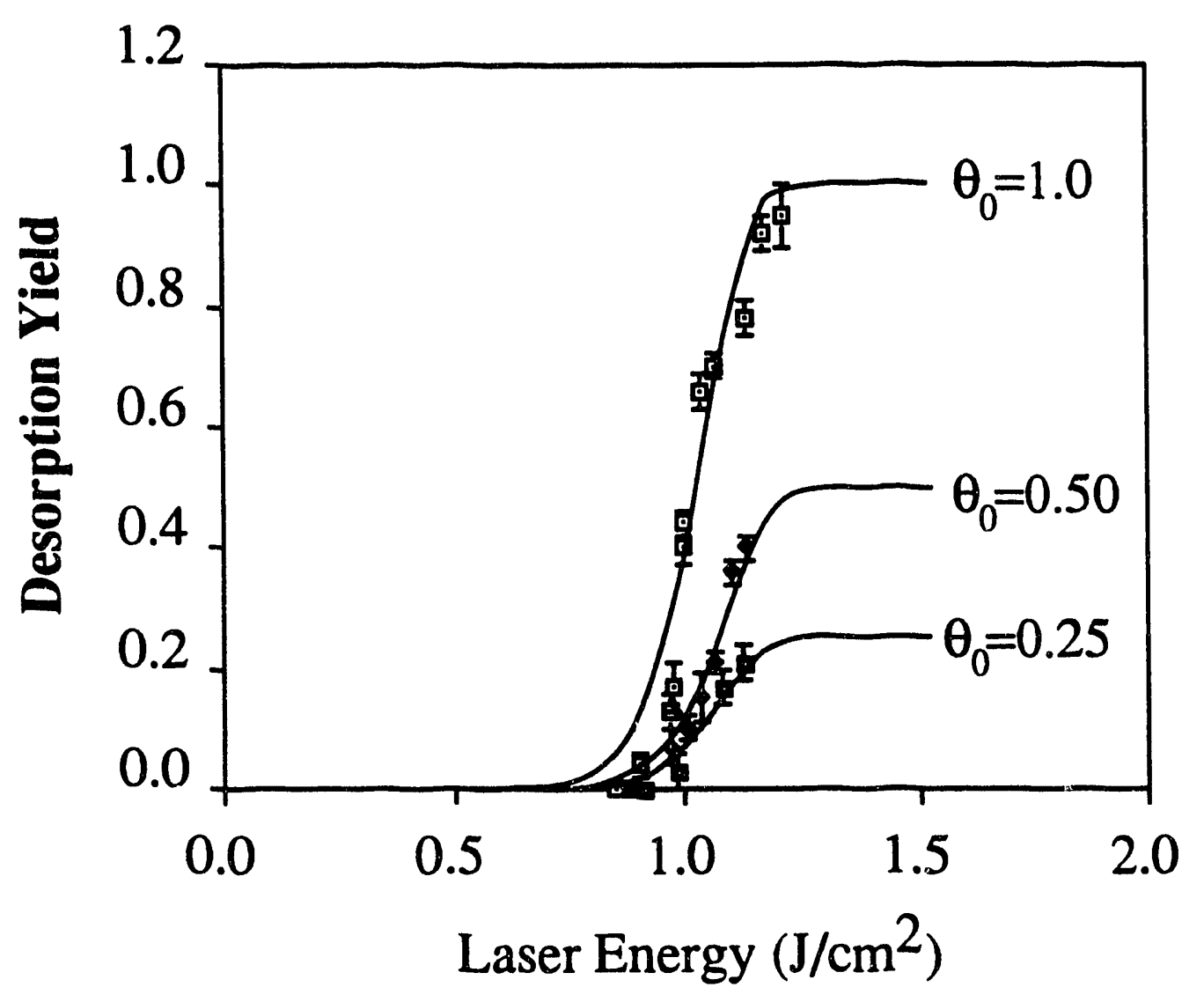

Figure 6 


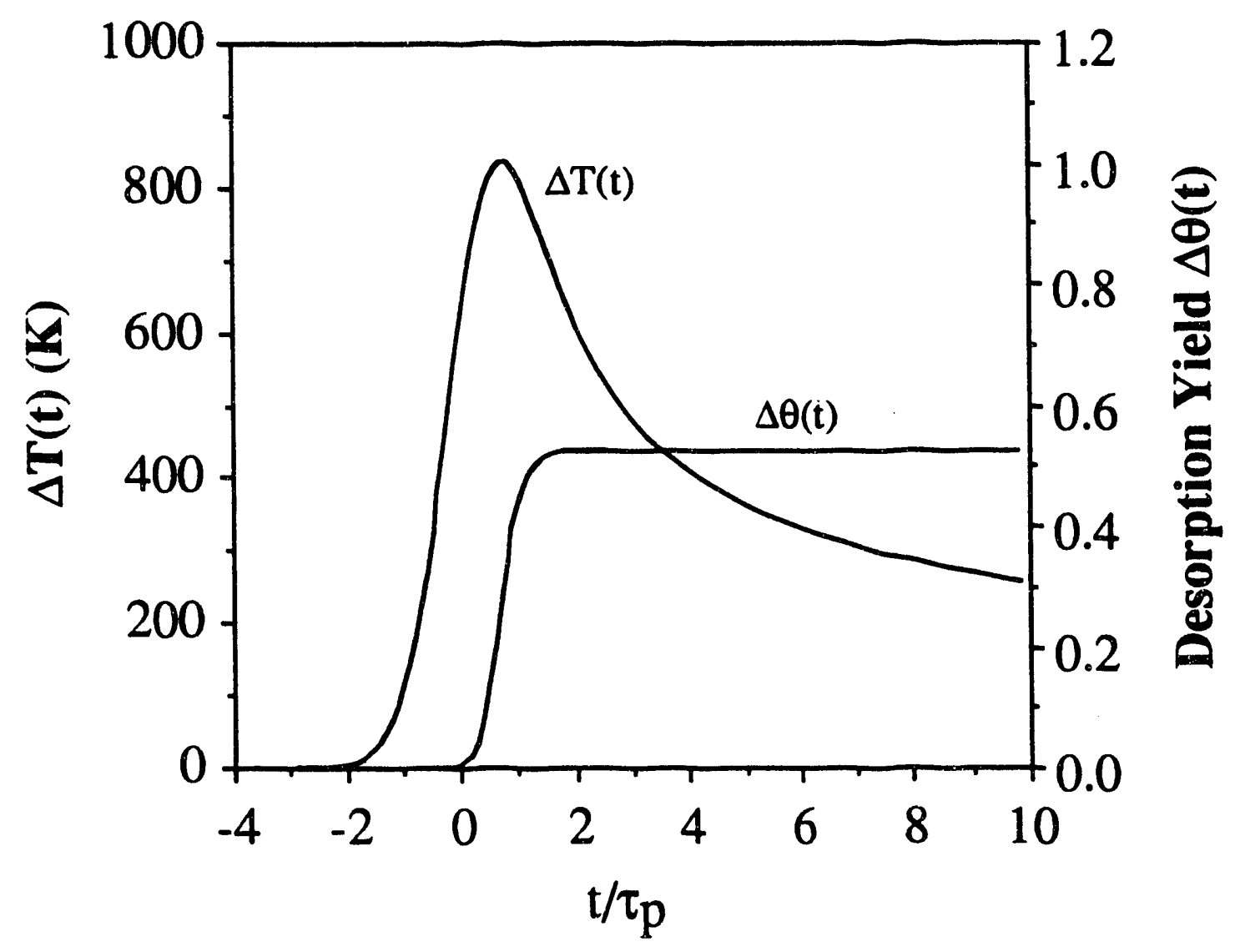

Figure 7 


\section{Vacuum Chamber}

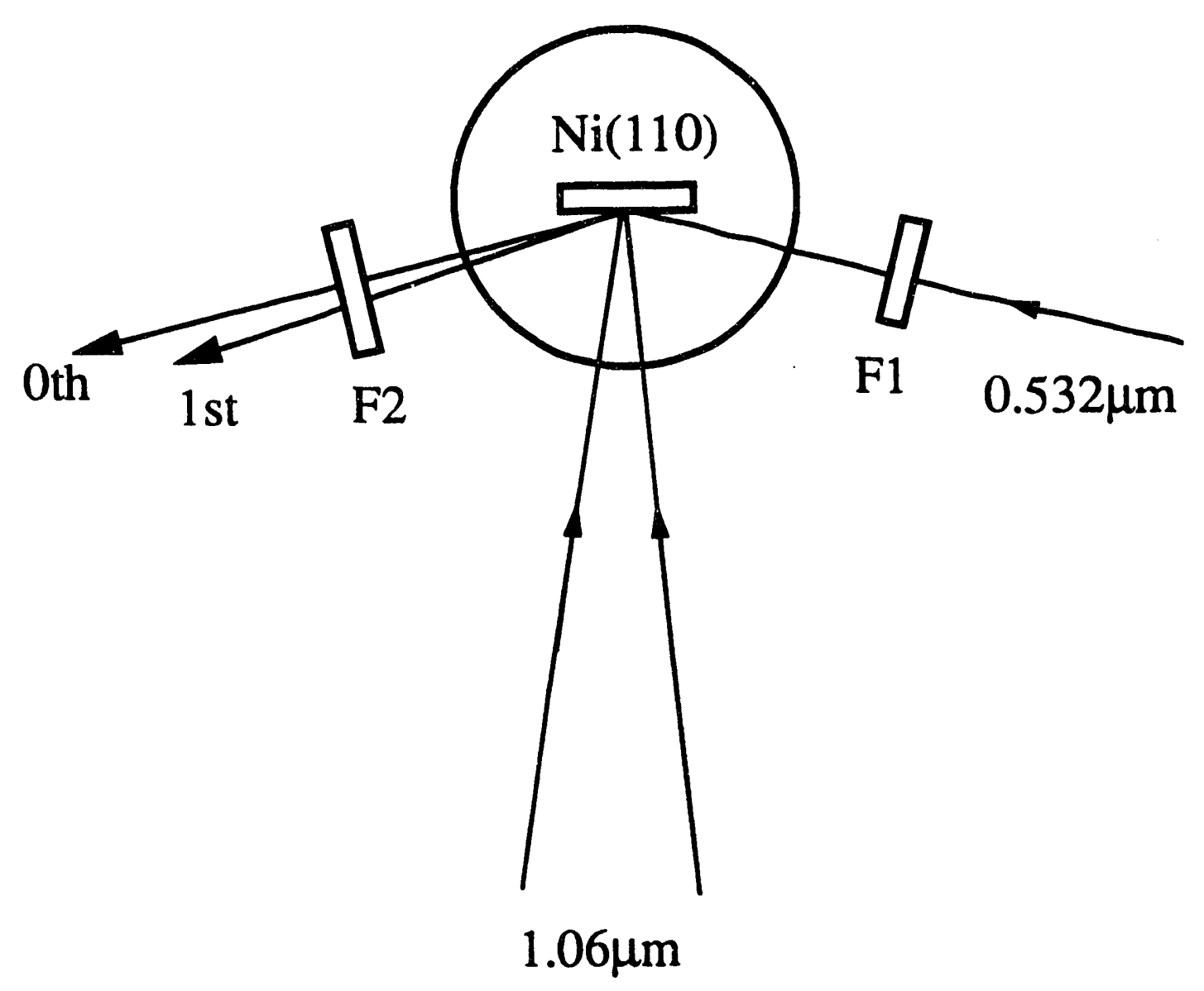

Figure 8 


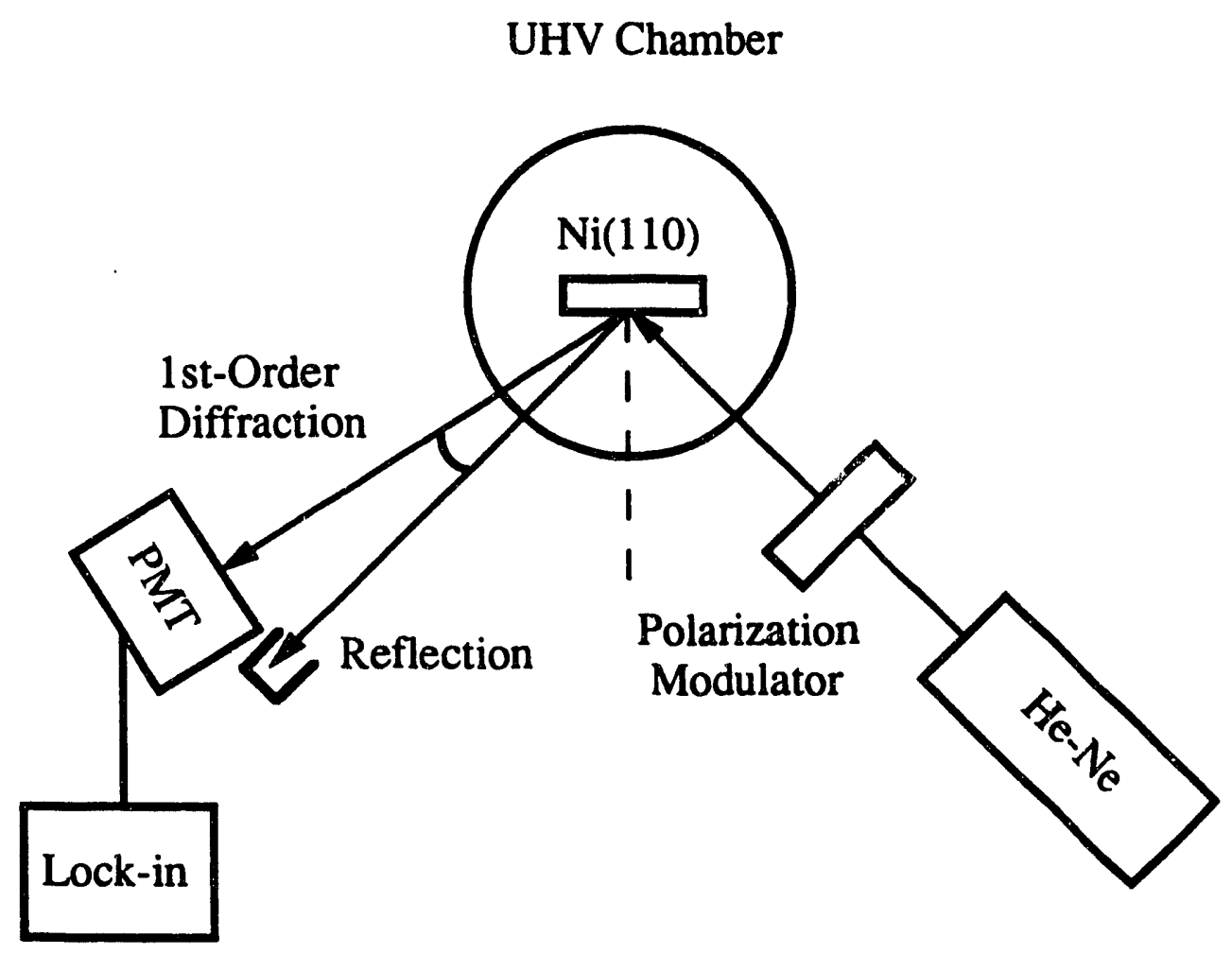

Figure 9 


\section{Optical Second Harmonic Diffraction Study of Anisotropic Surface Diffusion: CO on Ni(110)}

\section{A. Introduction}

The study of heterogeneous surface diffusion is a fundamental step towards understanding the mechanism of many surface processes, ranging from associative desorption of adsorbates, epitaxial crystal growth, to catalysis. $^{1,2,3}$ It can also provide useful information about the effective surface potential and diffusion pathways experienced by adsorbates. On crystalline surfaces, the structural anisotropy is expected to effect anisotropy in surface diffusion. Anisotropic surface diffusion can cause preferential development of surface reactions in ceriain forms and is therefore important in the practical consideration of controlling surface reactions. Surprisingly, despite its importance, research effort on anisotropic surface diffusion so far has been rather limited. This is presumably due to limitation in the existing experimental techniques.

In this chapter I will present an anisotropic surface diffusion study using the SH diffraction off a monolayer grating technique. As discussed in chapter III, this method is particularly suitable for surface diffusion anisotropy study. The system chosen to demonstrate the technique is CO/Ni(110).4 The Ni(110) surface has a row structure with atoms closely packed in the [1 $\overline{1} 0]$ direction (see Fig. 1). The $\mathrm{CO}$ molecules can adsorb with almost equal probabilities on both top and short-bridge sites up to a coverage of $\theta=0.85 .^{5}$ For $\theta>0.85$, the $C O$ molecules are pushed to the short-bridge site to form zig-zag chains along the [110] rows with adjacent CO molecules displaced in the [001] and [001] 
directions respectively ${ }^{6}$. At a full coverage, the tilt angle of $\mathrm{CO}$ molecular axis with respect to the surface normal is $\sim 20^{\circ}$ and the adsorbate structure appears as $2 \times 1 .^{6}$ Obviously, surface diffusion of $\mathrm{CO}$ on $\mathrm{Ni}(110)$ at all coverages must be anisotropic. At an average $\mathrm{CO}$ coverage of $\theta_{0} \sim 0.5$ we can expect diffusion along [110] as jumping from top or short-bridge sites to short-bridge or top sites; and along [001] as jumping from top sites to top sites and from shortbridge sites to short-bridge sites. Thus one would anticipate the existence of two independent diffusion channels, one along [110] and the other along [001] . They were indeed identified in our experiment. The diffusion energies and the pre-exponential factors for the two channels were deduced. Both of them show strong anisotropy, namely, the diffusion energy is significantly larger along [001] than along [1 $1 \overline{1} 0]$, accompanied by a larger pre-exponential factor also along [001]. Our results are however approximate since the coverage dependence of the diffusion coefficients has not been taken into account in the analysis. The coverage dependence of surface diffusion for $\mathrm{CO} / \mathrm{Ni}(110)$ will be the topic of next chapter.

\section{B. Experiment}

\section{a) Sample Preparation}

The experiment was performed in an ultrahigh vacuum (UHV) chamber with a base pressure of $1.0 \times 10^{-10}$ torr. The single crystal $\mathrm{Ni}(110)$ sample was cut and mechanically polished to within $0.3^{\circ}$ from the (110) plane with the miscut along the [001] direction, and mounted vertically on a rotatable sample holder capable of more than $90^{\circ}$ of rotation about [110]. Before any 
measurement, the surface of the sample was first $\mathrm{Ar}^{+}$sputtered (at $1.0 \times 10^{-4}$ torr with a $500 \mathrm{~V}$ beam voltage for approximately 30 minutes) at room temperature until no impurity contamination could be detected by the Auger electron spectrometer within its detection limit ( $<0.3 \%$ for carbon, and $<0.5 \%$ for sulfur). The sample was then annealed at $1070 \mathrm{~K}$ for a few seconds followed by a slow cooling down to the measurement temperature. Right before each dosing of $\mathrm{CO}$ the sample was flash heated to $570 \mathrm{~K}$ to remove residual adsorbed molecules from the ambient, mostly hydrogen and $\mathrm{CO}$. The adsorption of $\mathrm{CO}$ on $\mathrm{Ni}(110)$ surface was carried out at approximately $100 \mathrm{~K}$ by introducing $\mathrm{CO}$ into the chamber through a leak valve. A sharp $1 \times 1$ LEED pattern was observed for a clean $\mathrm{Ni}(110)$ surface and a $2 \times 1$ pattern for a full CO monolayer on $\mathrm{Ni}(110)$. In order to avoid possible alternation of the surface and the adsorbate monolayer by the electron beam in the LEED measurement, separately prepared monolayers were used for the diffusion experiment. A Chromel-Alumel thermal couple welded to the sample was used to monitor the sample temperature. The diffusion experiment was conducted in a temperature range of $100 \mathrm{~K}$ to $170 \mathrm{~K}$ and was controlled to within $2 \mathrm{~K}$. The average $\mathrm{CO}$ coverage for all the diffusion experiments at different temperatures and in different directions was $\theta_{0} \sim 0.5$,

with $\theta=1$ defined as full $\mathrm{CO}$ coverage with one $\mathrm{CO}$ molecule per $\mathrm{Ni}$ atom on the surface .

\section{b) Diffusion Measurement}

The optical arrangement for diffusion experiment has been shown in Fig.8 of chapter III. A single-mode Q-switched Nd:YAG laser with a pulse width of $10 \mathrm{~ns}$ at $1.06 \mathrm{~mm}$ was used for both the monolayer grating creation and the 
SH diffraction measurement. To create a $\mathrm{CO}$ monolayer grating on $\mathrm{Ni}(110)$, the $1.06 \mathrm{~mm}$ beam was split irito two and then recombined at incident angles of $\phi= \pm 1.50^{\circ}$ with an overlapping area of $\sim 2 \mathrm{~mm}$ in diameter on the $\mathrm{Ni}(110)$ surface fully covered by $\mathrm{CO}$. The grating period was $s=\lambda / 2 \sin \phi \sim 20 \mu \mathrm{m}$. The two beams' intensities were chosen so that the average intensity $I_{0}$ corresponded to a desorption yield of $0.5 \mathrm{ML}$, and the contrast of $r=0.53$ was enough to modulate the adsorbate from full coverage to zero coverage, making a square-wave-like pattern. This kind of grating could yield the highest SH diffraction signal. To probe the diffusion, a frequency-doubled laser beam at $0.532 \mu \mathrm{m}$ from the Nd:YAG laser was used. Its intensity was $\sim 1 / 10$ that of the desorbing beam. The beam was incident at $70^{\circ}$ with respect to the surface normal and the first-order second harmonic $(\mathrm{SH})$ diffraction from the $\mathrm{CO}$ grating was detected as a function of time in order to probe the decay of the $\mathrm{CO}$ grating via $\mathrm{CO}$ diffusion. The probe beam was not strong enough to desorb $\mathrm{CO}$ from $\mathrm{Ni}$, as could be checked by moritoring SHG in the specularly reflected direction. Alternatively, this was made sure by creating a $\mathrm{CO}$ adsorbate grating in the [001] direction at $\sim 100 \mathrm{~K}$ and monitoring the change in the first-order SH diffraction. No change was found for several hours, indicating that diffusion, desorption, and adsorption of $\mathrm{CO}$ are all negligible in that circumstance. The diffusion coefficient could be deduced from the decay of the diffracted SH signal.

In comparison with $\mathrm{CO} / \mathrm{Ni}(111)$ diffusion, the CO-induced SHG from $\mathrm{Ni}(110)$ is $4 \sim 5$ times smaller than that from $\mathrm{Ni}(111)^{7,8,9}$. The desorption energy range is also very narrow for $\mathrm{CO}$ on $\mathrm{Ni}(110)$ as opposed to $\mathrm{CO}$ on $\mathrm{Ni}(111)^{10}$. In the present case, the desorption laser energy has to be controlled to within $2 \sim 3 \%$ of $1.24 \mathrm{~J} / \mathrm{cm}^{2}$ in order to create a good grating that can yield a reasonable $\mathrm{SH}$ diffraction level ( 80 counts $/ 5 \mathrm{~min}$, with $\mathrm{S} / \mathrm{N} \sim 10$ in our measurement). These reasons make the surface diffusion experiment of $\mathrm{CO} / \mathrm{Ni}(110)$ rather difficult. 
Moreover, for gratings along different crystalline orientations, the SH diffraction signal may differ by about $10 \%$ because the $p$-in/p-out $\mathrm{SH}$ response with the plane of incidence parallel to different crystalline directions involve different components of the second-order nonlinear susceptibility tensor $\chi^{(2)}(\theta)$. For instance, $\chi_{e f f}^{(2)}$ is a linear combination of $\chi_{z z z}^{(2)}, \chi_{x z x}^{(2)}$, and $\chi_{z x x}^{(2)}$ if the plane of incidence is parallel to $[1 \overline{1} 0]$ and a linear combination of $\chi_{z z z}^{(2)}, \chi_{y z y}^{(2)}$, and $\chi_{z y y}^{(2)}$ if the plane of incidence is parallel to [001]. Nevertheless, this magnitude difference would not affect the diffusion coefficient measurement since it affected the signal strength but not the decay time constant, which is directly related to the diffusion coefficient as has been seen in Chapter III.

The diffusion anisotropy was measured in the following way. For measurement of $\mathrm{CO}$ diffusion along a selected direction on $\mathrm{Ni}(110)$, the sample was rotated to have that direction in the plane of incidence of the desorbing laser beams. The $\mathrm{CO}$ monolayer grating on the surface could then be created by the method described in Chapter III. The diffusion measurement along such a chosen direction was subsequently carried out at a few temperatures in order to find the temperature dependence of the diffusion coefficient $D(T)$. Measurements were performed for $\mathrm{CO}$ diffusion not only along the principal axes of $\mathrm{Ni}(110)$ but also along other directions of interest.

\section{Relations Between Diffusion Coefficient and the SH Diffraction Signal}

Surface diffusion is generally characterized by a rank-2 diffusion coefficient tensor $\overleftrightarrow{\mathbf{D}}$ which is related to the particle flux $\mathbf{J}$ and the surface 
concentration $\mathrm{C}$ by

$$
J=-\overleftrightarrow{\mathbf{D}} \cdot \nabla C
$$

The tensor can be diagonalized along symmetry axes in the surface. For the case of $\mathrm{Ni}(110)$ the axes for diagonalization are [170] and [001] , so that we have

$$
\overleftrightarrow{\mathbf{D}}=\left(\begin{array}{cc}
D_{[1 \overline{1} 0]} & 0 \\
0 & D_{[001]}
\end{array}\right)
$$

Thus for surface diffusion along a direction at an angle $\phi$ away from [1 $1 \overline{10}]$, the diffusion coefficient is given by

$$
D(\phi)=D_{[1 \overline{10}]} \cos ^{2} \phi+D_{[001]} \sin ^{2} \phi
$$

As discussed in the Chapter III, we are interested in observing surface diffusion from the time-dependent smearing of a monolayer grating of adsorbates. In this case, surface diffusion is governed by the one-dimensional diffusion equation

$$
\frac{\partial \theta}{\partial t}=\frac{\partial}{\partial x}\left(D \frac{\partial \theta}{\partial x}\right)
$$

with a periodic initial condition. In the case of coverage in.jependent $D$ the solution is 


$$
\begin{aligned}
\theta(x, t) & =\theta_{0}+\sum_{n=1}^{\infty} \theta_{n}(t) \cos (2 n \pi x / s) \\
& =\theta_{0}+\sum_{n=1}^{\infty} \theta_{n}^{0} \cos (2 n \pi x / s) \exp \left(-4 n^{2} \pi^{2} D t / s^{2}\right),
\end{aligned}
$$

where $\theta_{n}^{\circ}$ are constants. More generally, $D$ depends on $\theta$ and the solution becomes more complicated. This will be discussed later in the discussion section.

The nonlinear susceptibility $\chi_{\text {eff }}^{(2)}(\theta)$ responsible for SHG from a CO covered $\mathrm{Ni}(110)$ surface can be separated into two parts, one from the bare metal substrate, and the other from the adsorbate-induced contribution which depends on coverage:

$$
\chi_{e f f}^{(2)}(\theta)=\chi_{e f f}^{(2)}(0)+\Delta \chi_{e f f}^{(2)}(\theta)
$$

If $\theta(x)$ is periodic in $x$, then $\chi_{e f f}^{(2)}(\theta)$ is also periodic in $x$, and can be written as

$$
\chi_{\text {eff }}^{(2)}(\theta(x))=\chi_{\text {eff }}^{(2)}(0)+\sum_{n=0}^{\infty} A_{n}(t) \cos (2 n \pi x / s)
$$

with

$$
A_{n}=\lim _{L \rightarrow \infty} \frac{2}{L} \int_{-L / 2}^{L / 2} \chi_{\text {eff }}^{(2)}(\theta(x)) \exp \left(i \frac{2 n \pi x}{s}\right) d x .
$$


SHG from such a susceptibility grating appears both in specular reflection and in diffraction. The specularly reflected $\mathrm{SH}$ signal is proportional to $\left|x_{\text {eff }}^{(2)}(0)+A_{0}\right|^{2}$ and the nth-order diffracted SH signal is proportional to $\left|A_{n}\right|^{2}$. Consider the simple case that $\Delta \chi_{\text {eff }}^{(2)}(\theta)$ is linearly proportional to the coverage $\theta$. Then $A_{n} \propto \theta_{n}$ and the nth-order $\mathrm{SH}$ diffraction is given by

$$
\begin{aligned}
S_{n}(t) & \propto\left|\theta_{n}\right|^{2} \\
& =S_{0 n} \exp \left(-8 \pi^{2} n^{2} D t / s^{2}\right),
\end{aligned}
$$

From the time constant of the exponential decay of the diffracted SH signal, the diffusion coefficient $D$ can be deduced. Note that the decay time constant is independent of the grating pattern except the grating spacing s. If $\Delta \chi_{\theta f f}^{(2)}(\theta)$ is not linearly proportional to $\theta$, the situation again becomes more complicated as $S_{n}(t)$ is no longer proportional to $\left|\theta_{n}(t)\right|^{2}$. The decay of $S_{n}(t)$ would appear as multi-exponential. This will be discussed in the discussion section.

\section{Experimental Results}

The measured data of the first-order SH diffraction signal versus time from $\mathrm{CO}$ monolayer gratings on $\mathrm{Ni}(110)$ along three different directions, [110] $\left(\phi=0^{\circ}\right),[001]\left(\phi=90^{\circ}\right)$, and $\phi=45^{\circ}$, are presented in Fig. 2(a), 2(b), and 2(c), respectively. Assuming that Eq. (9) is valid, we fit the data at each temperature by a single exponential, as shown by the solid curves in Fig. 2. From the fit and using Eq. (9) with $n=1$, we can deduce the decay time constant and hence the 
diffusion coefficient $D(T)$ (with $s=20 \mu \mathrm{m}$ ). The fluctuation of the data points was mainly due to the poor signal-to-noise ratio. The uncertainty in determining the diffusion coefficient $D$ is around $\pm 40 \%$.

The deduced diffusion coefficient $D$ versus $1 / T$ is plotted in Fig. 3 for $C O$ diffusion along the three specific directions on $\mathrm{Ni}(110)$. The results for diffusion along the orthogonal directions, [110] and [001], are well described by the Arrhenius form,

$$
D=D_{0} \exp \left(-E_{d i f f} / k_{B} T\right)
$$

This indicates that $\mathrm{CO}$ diffusion on $\mathrm{Ni}(110)$ has two distinct channels, one along [110] and the other along [001]. The fit of Eq. (10) to the data points in Fig. 3 yields

$$
\begin{aligned}
& \text { along [110]: } E_{\text {diff }}([1 \overline{1} 0])=1.1 \pm 0.2 \mathrm{kcal} / \mathrm{mol},(0.048 \mathrm{eV}) \\
& D_{0}([1 \overline{1} 0])=(3.8 \pm 2.0) \times 10^{-9} \mathrm{~cm}^{2} / \mathrm{sec} \text {; } \\
& \text { along [001]: } E_{\text {diff }}([001])=3.1 \pm 0.4 \mathrm{kcal} / \mathrm{mol},(0.134 \mathrm{eV}) \\
& D_{0}([001])=(4.8 \pm 4.4) \times 10^{-6} \mathrm{~cm}^{2} / \mathrm{sec} \text {. }
\end{aligned}
$$

That surface diffusion of $\mathrm{CO}$ on $\mathrm{Ni}(110)$ is anisotropic is obvious from the above results. For diffusion along the direction $\phi=45^{\circ}$, we expect from Eq. (3)

$$
D\left(\phi=45^{\circ}\right)=\frac{1}{2}\left(D_{[1 \overline{1} 0]}+D_{[001]}\right)
$$


Plotted in Fig. 3, Eq. (11) fits the experimental data very well. Note that two combined exponential functions of $1 / \mathrm{T}$ are needed to describe $D\left(\phi=45^{\circ}\right)$. This further supports the picture of two orthogonal independent diffusion channels of $\mathrm{CO}$ on $\mathrm{Ni}(110)$.

Figure 4 depicts the measured $\mathrm{D}$ as a function of the diffusion direction specified by $\phi$ at fixed temperature T 110K. The solid curve calculated from Eq. (3) is also in good agreement with the data. The diffusion anisotropy at $T=110 \mathrm{~K}$ is obviously very significant.

\section{E. Discussion}

In deducing the diffusion coefficient $D$ from our experiment, we have made a number of simplifying assumptions (see Sec. C). In this section, we shall first consider the effects of those assumptions and other possible experimental complications before we discuss the implication of the experimental results.

\section{a) Heating Effect From the Probing Beam}

One may wonder if the laser beam used to probe the monolayer grating would heat up the sample surface and significantly affect the surface diffusion of the adsorbates. In our measurements, the fluence of the probe laser pulse was $\sim 0.1 \mathrm{~J} / \mathrm{cm}^{2}$. Using Eq. (9) in Chapter III, we estimate a maximum temperature rise of $65 \mathrm{~K}$ for the $\mathrm{Ni}$ surface at $t \sim 10 \mathrm{nsec}$ due to laser heating. This temperature rise decays away to $\Delta T<10 \mathrm{~K}$ at $t \sim 40$ nsec. From Eq. (10), we find $D(T+\Delta T) / D(T)$ 
$=10$ to 500 for a temperature range of $T \sim 100$ to $170 \mathrm{~K}$ with $\Delta T=65 \mathrm{~K}$ if $E_{\text {diff }}=3.1$ $\mathrm{kcal} / \mathrm{mol}$. The excess mean square displacement resulting from $D(T+\Delta T)$ during $\delta \mathrm{t}$

the heating period of $\delta t \sim 40$ nsec is given by $\Delta<x^{2}>=\int_{0} 2\{D(T+\Delta T)-D(T)\} d t$ $\sim 2 \mathrm{D}(\mathrm{T}+\Delta \mathrm{T}) \delta \mathrm{t}$. This is negligible compared to the mean square displacement $2 D(T) \Delta t$ of $C O$ molecules during the period $\Delta t=0.1 \mathrm{sec}$ between two successive laser pulses. For smaller $E_{\text {diff, }}$ the effect is even smaller. Thus we can conclude that the probe laser heating effect is insignificant in our surface diffusion measurements.

\section{b) Coverage Dependence of Nonlinear Susceptibility}

In the data analysis, we assumed $\Delta \chi_{e f f}^{(2)}(\theta)$ is linear in $\theta$. This is not true in general and is a poor approximation for $\mathrm{CO}$ on $\mathrm{Ni}(110)$ as seen in Fig. 2 of Chapter III. As mentioned briefly in Sec. $C$, the nonlinear relation between $\Delta \chi_{\text {eff }}^{(2)}(\theta)$ and $\theta$ may cause the first-order SH diffraction to decay multiexponentially or non-exponentially. This can be seen by expanding $\Delta \chi_{\theta f f}^{(2)}(\theta)$ into power series of $\left(\theta-\theta_{0}\right)$, where $\theta_{0}$ is the average surface coverage of the monolayer grating.

$$
\Delta \chi_{e f f}^{(2)}(\theta)=\Delta \chi_{e f f}^{(2)}\left(\theta_{0}\right)+\left(\frac{d \Delta \chi_{e f f}^{(2)}}{d \theta}\right)_{\theta_{0}}\left(\theta-\theta_{0}\right)+\frac{1}{2}\left(\frac{d^{2} \Delta \chi_{\theta f f}^{(2)}}{d \theta^{2}}\right)_{\theta_{0}}\left(\theta-\theta_{0}\right)^{2}+
$$




$$
+\frac{1}{6}\left(\frac{d^{3} \Delta \chi_{\theta f f}^{(2)}}{d \theta^{3}}\right)_{0}\left(\theta-\theta_{0}\right)^{3}+\ldots
$$

From Eq. (8), the first-order SH diffraction amplitude takes the form

$$
\begin{aligned}
A_{1}(t) & =\left(\frac{d \Delta \chi_{\text {eff }}^{(2)}}{d \theta}\right)_{\theta_{0}} \theta_{1}{ }^{0} \exp (-t / 2 \tau)+ \\
& +\frac{1}{2}\left(\frac{d^{2} \Delta \chi_{\text {eff }}^{(2)}}{d \theta^{2}}\right)_{\theta_{0}} \sum_{m=1} \theta_{m}^{0} \theta_{m+1}^{0} \exp \left(-\left[m^{2}+(m+1)^{2}\right] t / 2 \tau\right) \\
& +\frac{1}{8}\left(\frac{d^{3} \Delta \chi_{e f f}^{(2)}}{d \theta^{3}}\right)_{0} \sum_{(m, n)=1} \theta_{m}^{0} \theta_{n}^{0} \theta_{m+n+1}^{0} \exp \left(-\left[m^{2}+n^{2}+(m+n+1)^{2}\right] t / 2 \tau\right) \\
& \left.+\sum_{(m, n)=1} \theta_{m} \theta_{n} \theta_{m+n-1} \exp \left(-\left[m^{2}+n^{2}+(m+n-1)^{2}\right] t / 2 \tau\right)\right\} \\
& +\ldots \ldots . \quad, \quad(13)
\end{aligned}
$$

where $\tau=s^{2} / 8 \pi^{2} D$ and $D$ is assumed constant. Since generally, $\theta_{m+1}^{0}<\theta_{m}^{0}$

$<1 / 2$ for $m>1$, we expect that the higher-order terms can be appreciably smaller than the first term in Eq. (13). The two leading correction terms are $\frac{1}{2}\left(d^{2} \Delta \chi_{\text {eff }}^{(2)} / d \theta^{2}\right)_{\theta_{0}} \theta_{1}^{0} \theta_{2}^{0} \exp (-5 t / 2 \tau)$, and $\frac{1}{8}\left(d^{3} \Delta \chi_{\text {eff }}^{(2)} / d \theta^{3}\right) \underset{\theta_{0}}{\left(\theta_{1}^{0}\right)^{3}}$ $x \exp (-3 t / 2 \tau)$. For $t>\tau / 2$, they are further reduced by factors larger than $e^{-5 / 4}$ and $e^{-3 / 4}$, respectively. Thus we can conclude that if $\Delta \chi_{e f f}^{(2)}(\theta)$ can be 
approximated by a power series expansion of Eq. (12) and if the data analysis puts more emphasis on the diffraction data at later times, then a singleexponential decay of the diffraction with $\Delta \chi_{\theta f f}^{(2)}(\theta)=\Delta \chi_{\theta f f}^{(2)}\left(\theta_{0}\right)+\left(d \Delta \chi_{\theta f f}^{(2)} / d \theta\right)_{\theta_{0}}$ $x\left(\theta-\theta_{0}\right)$ is a fair approximation. In principle, one can make grating groove sufficiently shallow to render $\Delta \chi_{\text {eff }}^{(2)}(\theta) \propto\left(\theta-\theta_{0}\right)$ so that the decay would certainly be single exponential. Unfortunately, limited by the diffraction signal strength which is proportional to $\left|\Delta \chi_{\text {eff }}^{(2)}(\theta)\right|^{2}$, this may not always be possible.

For the case of $\mathrm{CO}$ on $\mathrm{Ni}(110)$, the experimental data of $\Delta \chi_{\text {eff }}^{(2)}(\theta)$ can be approximated by (see Fig. 2 of Chapter III)

$\Delta \chi \underset{\text { eff }}{(2)}(\theta) \propto 0.707+0.707\left(\theta-\theta_{0}\right)-0.354\left(\theta-\theta_{0}\right)^{2}+0.354\left(\theta-\theta_{0}\right)^{3}+\ldots$,

with $\theta_{0}=0.5$. If we assume an initial $C O$ monolayer grating of the rectangular periodic form

$$
\theta(x)=0.5+\sum_{n=1}^{\infty} \frac{2}{n \pi} \sin \left(\frac{n \pi}{2}\right) \cos (2 n \pi x / s)
$$

Then we can show from Eq. (13) that by keeping only the first term in Eq. (13), the decays calculated from $\left|A_{1}(t)\right|^{2}$ with $t \geq 0, t \geq \tau / 2$, and $t \geq \tau$ are $22 \%, 4 \%$, and $1 \%$ slower than the real case.

In deducing $D$ from fitting our experimental data with $\left|A_{1}(t)\right|^{2}$, we recognized the poor signal-to-noise ratio at large $t$. We therefore fit the data with a single exponential starting from $t=0$, knowing that the deduced value of $D$ could be larger than the real value by about $22 \%$. This is especially true for the 
lower temperature cases, where less data with $t>\tau / 2$ are available because of larger $\tau$. These systematic errors make our deduced diffusion activation energies and pre-exponential factors somewhat smaller than their real values.

\section{c) Coverage Dependence of Diffusion Coefficient}

The diffusion coefficient generally also depends on the surface coverage of adsorbates which we have neglected in our data analysis. If the dependence of $D$ on $\theta$ is strong and the grating groove depth is deep, then even with $\Delta \chi_{\text {eff }}^{(2)}(\theta)$ linear in $\theta$, the first-order SH diffraction will not have a single exponential decay. This is seen as follows.

Assume $D(\theta)$ can be described by a power series

$$
D(\theta)=D\left(\theta_{0}\right)\left[1+d_{1}\left(\theta-\theta_{0}\right)+d_{2}\left(\theta-\theta_{0}\right)^{2}+\ldots\right] .
$$

From Eqs. (4) and (5), we find,

$$
\begin{aligned}
& \frac{\partial \theta_{1}}{\partial t}=-\frac{\pi^{2}}{a^{2}} D\left(\theta_{0}\right)\left[\theta_{1}+\frac{1}{2} d_{1} \theta_{1} \theta_{2}+\frac{1}{4} d_{2} \theta_{1}^{3}+\ldots\right] \\
& \frac{\partial \theta_{2}}{\partial t}=-\frac{4 \pi^{2}}{a^{2}} D\left(\theta_{0}\right)\left[\theta_{2}+\frac{1}{4} d_{1} \theta_{1}{ }^{2}-\frac{11}{8} d_{1} \theta_{1} \theta_{3} \ldots\right] .
\end{aligned}
$$

It is obvious that the solution of Eq. (17) will give a $\theta_{1}(t)$ with a non-exponential or multi-exponential decay. Thus even if $\Delta \chi_{\text {eff }}^{(2)}(\theta) \propto\left(\theta-\theta_{0}\right)$ so that $A_{1}(t)=$ 
$\left(d \Delta x_{\theta f f}^{(2)} / d \theta\right)_{\theta_{0}} \theta_{1}(t)$ from Eq. (13), the first-order SH diffraction may decay non-exponentially. However, if $d_{1} \theta_{1} \theta_{2}$ and the higher-order terms in Eq. (17) are much smaller than $\theta_{1}$, we still have

$$
\frac{\partial \theta_{1}}{\partial t} \cong-\frac{\pi^{2}}{a^{2}} D\left(\theta_{0}\right) \theta_{1}
$$

and hence $\theta_{1}(t) \propto \exp (-t / 2 \tau)$, from which $D\left(\theta_{0}\right)$ can be deduced. This can be achieved with a sufficiently small $\theta_{2}$ either from a shallow monolayer grating with a small initial $\theta_{2}^{0}$ or by waiting long enough for $\theta_{2}$ to decay to a small value.

In our experiment, the $\mathrm{CO}$ monolayer grating was square-wave-like with a modulation ranging from zero to full coverage. We estimated $\theta_{2}^{0} \sim 0.1$. The fact that the decay of SH diffraction can be roughly fit by single exponentials suggests $d_{1} \ll 20$ and $d_{2} \ll 4$. The values of $D\left(\theta_{0}\right)$ deduced from the experiment are accurate to within a factor of 5 judging from the above discussion.

As we will see in Chapter $V$, the diffusion coefficient has a strong coverage dependence and therefore with a deep modulation on the coverage grating our data analysis can only provide some kind of effective diffusion coefficient $D$. What is the meaning of this $D$ ? Is it the diffusion coefficient at the average coverage? To answer these questions, let us use a coverage dependent $D(\theta)$ given by

$$
D(\theta)=D(0), \quad \text { for } \theta<c
$$




$$
=D(0) \exp \left(d(T) \frac{\theta-c}{1-c}\right), \quad \text { for } \theta>c
$$

where $d(T)$ is a temperature dependent constant and decreases as temperature increases. As we will see in Chapter V, this coverage dependent diffusion coefficient $\mathrm{D}(\theta)$ can describe $\mathrm{CO}$ diffusion on $\mathrm{Ni}(110)$ with $c=0.67$ quite well. With this $D(\theta)$, we have solved the diffusion equation (Eq. (4)) numerically for an initial square-wave coverage profile. The evolution of the coverage is shown in Fig. 5(a) for $d=2.5$. It is clear from this graph that the high coverage region smears out significantly faster than the low coverage region, especially in the early time period $\left(k<s^{2} / 8 \pi^{2} D(\theta=0.9)\right)$. The calculated $S H$ diffraction signal for $d=2.5$ ( Eq. (8)) by taking Eq. (14) for $\Delta \chi_{\text {eff }}^{(2)}(\theta)$ is depicted in Fig. $5(\mathrm{~b})$. The diffusion coefficient obtained is $\sim 3.5 \mathrm{D}(0)$ if we limit the approximate data fitting to decay to only $70 \%$ of the initial signal and is $\sim 1.25 \mathrm{D}(0)$ if we fit the data approximately down to $5 \%$ of the initial signal. The physical picture here is that in the early time period, diffusion occurs mostly in the high coverage region and therefore the diffusion coefficient deduced from the SH signal corresponds to high coverage values. As time goes on, the weighting of the lower coverage $(\theta<0.7)$ diffusion becomes larger and larger and brings the deduced diffusion coefficient close to low coverage values. This result clearly demonstrates that $D$ deduced from our measurement, in general, is neither the diffusion coefficient at the average coverage (which should be $D(0)$ in the above case) nor that of a unique effective coverage. Depending on the length of the relative time $t / \tau$ $\left(\tau=s^{2} / 8 \pi^{2} D(0)\right)$ in which the data has been collected, the deduced $D$ may correspond to the value at a very high coverage (if $t / \tau<<1$ ) or to that at a somewhat lower coverage (if $t / \tau \gg 1$ ). In particular, the deduced diffusion coefficient in our experiment at low temperatures appears to correspond to the 
value at high coverages ( $0.90 \mathrm{ML})$ since only data during the initial decay period $t \ll \tau$ were collected, while at high temperatures it should correspond to a value at relatively low coverages $(\sim 0.7 \mathrm{ML})$ since data with $t \gg \tau$ were measured.

Guided with this general argument and using the coverage dependent diffusion coefficients for diffusion along [1 10$]$ from Chapter $V$, we have obtained the effective $D$ as a function of reciprocal temperature and the results are shown in Fig. 6(a). It is seen that the diffusion activation energy deduced from such a set of data can appear lower than the real activation energy at full coverage. This is indeed the case for $E_{\text {diff }}[1 \overline{1} 0]$ observed in our SHG experiment, which is $1.1 \mathrm{kcal} / \mathrm{mol}$ as compared to $2.0 \mathrm{kcal} / \mathrm{mol}$ at full coverage. Therefore, the diffusion parameters $E_{\text {diff }}$ and $D_{0}$ determined from the experiment are not quantitatively meaningful.

However, unlike the case of diffusion along [110], the activation energy for $\mathrm{CO}$ diffusion along [001] deduced from the present set of clata is still comparable to the value at full coverage (Chapter V), with the former being $3.1 \mathrm{kcal} / \mathrm{mol}$ and the latter being $2.8 \mathrm{kcal} / \mathrm{mol}$. This can be understrod if we notice that the measured SH signal $\left|\chi_{\text {eff }}^{(2)}(\theta)\right|^{2}=\left|\chi_{\text {eff }}^{(2)}(0)+\Delta \chi_{\text {eff }}^{(2)}(\theta)\right|^{2}$ along [001] direction is insensitive to CO coverage above $0.80 \mathrm{ML}$ (Fig. 7). Using a $\Delta \chi_{e f f}^{(2)}(\theta)$ given by Eq. (14) below $0.8 \mathrm{ML}$ and by a constant above $0.80 \mathrm{ML}$, simulation of $\mathrm{CO}$ diffusion with an initial square-wave coverage profile yields approximate diffusion coefficients at low temperatures corresponding to $\theta<0.8 \mathrm{ML}$ (Fig. 6(b)). Because the SH diffraction is insensitive to diffusion of high coverages, the measured $E_{\text {diff }}[001]$ appears to be somewhat larger than the activation energy at full coverage. 
In principle, we should be able to quantitatively simulate the $\mathrm{SH}$ diffraction resulis by the experimentally measured $D(\theta)$ from Chapter $V$. Unfortunately, we have not succeeded in doing so. A number of reasons could be responsible for it. First, the accuracy of $D(\theta)$ was not sufficient for such a quantitative simulation and the functional form of $D(\theta)$ given by Eq. (18) was only meant as an approximation. Second, the initial coverage of each grating prepared for SH diffraction experiment was not necessarily the same and their detail shapes could have affected the decay constants of SH diffraction differently at different temperatures. Third, by no means the form of $\chi_{\text {eff }}^{(2)}(\theta)$ used in the simulation was accurate enough. Fourth, and the most importantly, the present measurements were carried out on a different $\mathrm{Ni}(110)$ surface from those presented in Chapter V. With significantly stronger laser intensities used to create the adsorbate gratings in the present measurements, the $\mathrm{Ni}(110)$ surface could be disturbed to a higher degree. As we will see in Chapter V and VI, laser-induced defects may have significant effect on diffusion.

\section{d) Effects of Surface Defect}

Before we discuss the results of our surface diffusion measurements, we need to know whether they are intrinsic to the $\mathrm{Ni}(110)$ surface or dominated by defects on the surface. First, consider the effect of point defects. Their density is presumably around $10^{-3}$ to $10^{-4}$ of a full monolayer if it is properly annealed. ${ }^{11}$ These defect sites are often first covered by adsorbates because of the stronger binding energy. In our experiment with an average coverage of $\theta_{0} \sim 0.5$, the effect of such point defects may be negligible. The same argument can apply to 
short line defects (ineffective in blocking diffusion paths) with lengths much shorter than the size of the grating. (However, I will present experimental evidence in Chapter VI that renders above statements).

Special attention has to be paid to line defects which run across the sample and are parallel to the adsorbate grating. They can be steps arising from a miscut of the sample. For diffusion perpendicular to the steps, we have to consider the durations that the adsorbate molecules spend on the terraces and in traversing the steps. Let the average trapping times of an adsorbate molecule on a terrace and at a step site be $\tau_{T}$ and $\tau_{S}$, respectively. The total time for the molecule to diffuse across a terrace and a step is simply the sum

$$
\tau_{\text {tot }}=\tau_{T}+\tau_{S}
$$

If $\mathrm{Na}$ is the average width of a terrace, a the lattice constant and also the width of the steps, and $N$ the average number of rows of atoms in a terrace, then from $\left\langle x^{2}\right\rangle=2 D t$, we have $(N+1)^{2} a^{2}=2 D \tau_{\text {tot }}$. With $D_{T}$ and $D_{S}$ denoting the diffusion coefficients of adsorbates diffusing on a terrace and across a step, respectively, we also have $N^{2} a^{2}=2 D_{T} \tau_{T}$ and $a^{2}=2 D_{S} \tau_{S}$. We then find

$$
\frac{1}{D}=\frac{2 \tau_{\text {tot }}}{(N+1)^{2} a^{2}}=\frac{N^{2}}{(N+1)^{2}} \frac{1}{D_{T}}+\frac{1}{(N+1)^{2}} \frac{1}{D_{S}} \text {, }
$$

For the steps to dominate in the surface diffusion, we must have $D_{S} \ll D_{T} / N^{2}$.

In our case, the $\mathrm{Ni}(110)$ surface had a $0.3^{\circ}$ miscut along the [001] direction. This leads to an average terrace width of $\mathrm{N} 70$. If we assume that the trial frequencies (pre-exponential factors in $D$ ) for crossing a step and jumping over a barrier on a terrace are roughly the same, then $D_{S} \ll D_{T} / N^{2}$ leads to 


$$
\begin{aligned}
\gamma \equiv N^{2} D_{S} / D_{T} \\
=70^{2} \exp \left[\left(E_{\text {diff }}(\text { terrace })-E_{\text {diff }}(\text { step })\right) / k_{B} T\right] \ll 1
\end{aligned}
$$

where $\mathrm{E}_{\text {diff }}$ (terrace) and $\mathrm{E}_{\text {diff }}$ (step) are the diffusion activation energies on a terrace and across a step, respectively. If what we measured in our experiment were a step-dominated diffusion process $(\gamma<1)$ with $E_{\text {diff }}($ step $)=3.1 \mathrm{kcal} / \mathrm{mol}$ as obtained by fitting $D=D_{0} \exp \left(-E_{\text {diff }} / k_{B} T\right)$ to the diffusion data along [001], then Eq.(21) dictates $E_{\text {diff }}$ (terrace) should be smaller than $\left[E_{\text {diff }}\right.$ (step) - 8.5kT], which at $T=150 \mathrm{~K}$ is $0.56 \mathrm{kcal} / \mathrm{mol}$. This small value of $E_{\text {diff }}$ (terrace) is only twice as much as the thermal energy $(0.3 \mathrm{kcal} / \mathrm{mol})$ and would make the stable adsorption of $\mathrm{CO}$ on top and short-bridge sites of $\mathrm{Ni}(110)$ unlikely, contrary to the experimental observation. Therefore, we believe that the measured diffusion is intrinsic for $\mathrm{CO}$ on $\mathrm{Ni}(110)$ with $p>1$, and the effect of line defects is not significant. If we assume $\gamma=10$ we estimate from Eq. (21) that $E_{\text {diff }}$ (step) $\sim 6$ $\mathrm{kcal} / \mathrm{mol}$.

\section{e) Diffusion Results}

Despite the various systematical errors discussed above in the measurement, the diffusion results are very suggestive. First, under similar conditions for measurements along [110] and [001], for which similar systematical errors must exist, the anisotropy of the surface diffusion of $\mathrm{CO}$ on $\mathrm{Ni}(110)$ has been beautifully shown. Two independent diffusion barriers, one along [1 $1 \overline{1} 0]$ the other along [001], have been identified. To our best knowledge, 
this is the first direct observation of anisotropic heterogeneous molecular diffusion with two independent channels.

The two diffusion activation energies along [110] and [001] are clearly different, with the barrier along [001] higher than that along [110]. Qualitatively, this is understandable since the $\mathrm{Ni}$ atoms form closely packed rows along [1 $\overline{1} 0$ ] and the resulting surface potential seen by a $\mathrm{CO}$ which is adsorbed on the $\mathrm{Ni}$ rows is then expected to be less corrugated. A theoretical calculation by Doyen and Entl indeed predicted a surface potential variation of $\sim 1.25 \mathrm{kcal} / \mathrm{mol}$ along [110] and $\sim 2.25 \mathrm{kcal} / \mathrm{mol}$ along the [001] direction ${ }^{12}$. The experimental observation of streak-like $c(4 \times 2)$ and $c(8 \times 2)$ LEED patterns by Behm et al, which was interpreted as a consequence of $\mathrm{CO}$ occupation at intermediate positions other than the high symmetry sites (on-top and short-bridge sites) along [1 $1 \overline{10}$ ] direction. further indicated that the potential corrugation along [1 $\overline{1} 0]$ is smoother than that along $[001]^{13}$.

The deduced diffusion preexponential factors along the two directions are also anisotropic, however, the systematical errors may have contributed to it to some larger extent than to the diffusion activation energy. Therefore, any quantitative discussion on them is not very meaningful. The detail discussion of the implication of $\mathrm{CO}$ surface diffusion on $\mathrm{Ni}(110)$ should be postponed until better quality data are available (see Chapter V).

In comparison with adatom diffusion of metal atoms on the (110) plane of fcc crystals such as $\mathrm{Ni} / \mathrm{Ni}(110), \operatorname{Pt} / \mathrm{Pt}(110), \operatorname{Ir} / \mathrm{Pt}(110), \operatorname{Ir} / \operatorname{Ir}(110)$ and $W / \operatorname{Ir}(110)$, in which two distinct channels with adatoms hopping along the atomic rows of the substrate or exchanging with substrate atoms (concerted motion) to go across the rows ${ }^{3}$, we can conclude that the diffusion paths for $\mathrm{CO} / \mathrm{Ni}(110)$ are as follows: along the $[1 \overline{1} 0]$ direction, a CO molecule hops successively from a 
short-bridge (or on-top) site to a neighboring on-top (or short-bridge) site, then to a neighboring short-bridge (on-top) site, and so on; along the [001] direction, $\mathrm{CO}$ hops either from a short bridge site through a hollow site to another shortbridge site and so on or from a on-top site through a long-bridge site to another on-top site and so on (see Fig. 1).

\section{F. Conclusion}

The new technique using $\mathrm{SH}$ diffraction from a monolayer grating to measure surface diffusion is applicable to measure surface diffusion for all surfaces and is ideal for studying diffusion on crystalline surfaces with strong anisotropy. The anisotropic surface diffusion of $\mathrm{CO} / \mathrm{Ni}(110)$ is used here as a demonstration. The results indicate unequivocally the existence of two independent diffusion channels along [001] and [110] with strong anisotropy. The smaller activation energy for diffusion along [1 $1 \overline{1} 0]$ is directly associated

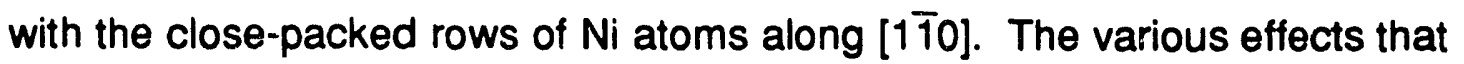
may influence the data analysis have been discussed.

As seen from the work described here, the monolayer grating technique has clearly the advantage of involving a simple and straightforward data analysis in the case with the optical field response linear to coverage and the diffusion coefficient independent of coverage. This eliminates the need of developing a theory just for the data analysis as with some other techniques. However, the present method using SH diffraction to probe the monolayer grating often suffers from a poor signal-to-noise ratio. This makes the study of , for example, coverage dependence of surface diffusion difficult. It is possible to greatly enhance the sensitivity by using linear optical diffraction instead to probe the monolayer grating. The coverage dependence of surface diffusion of 
$\mathrm{CO} / \mathrm{Ni}(110)$ which we have neglected in the present work can then be measured. This is the topic of next chapter. 


\section{References}

${ }^{1}$ An excellent review of the field has been provided recently by $R$. Gomer, Rep. Prog. Phys. 53, 917(1990).

2 A. G. Naumovets and Yu.S. Vedula, Surf. Sci. Reports 4, 365(1985).

${ }^{3}$ G. Ehlich and Kaj Stolt, Ann. Rev. Phys. Chem. 31, 603(1980).

${ }^{4}$ Xu-dong Xiao, X. D. Zhu, W. Daum and Y. R. Shen, Phys. Rev. Lett. 66, 2352(1991).

5 J. Bauhofer, M. Hock and J. Kuppers, Surf. Sci.191, 395(1987).

${ }^{6}$ D. J. Hannaman and M.A.Passler, Surf. Sci. 203, 449(1988); S.Haq, J.G.Love and D.A.King, Surf. Sci. 275, 170(1992); B. Voigtlander, S. Lehwald and H. Ibach, Surf. Sci. 225, 151(1990).

7 X. D. Zhu, Winfried Daum, Xu-dong Xiao, R. Chin and Y. R. Shen, Phys. Rev. B 43, $11571(1991)$.

${ }^{8}$ X. D. Zhu, and Y. R. Shen, Opt. Lett. 14, 503(1989).

9 J. C. Hamilton, R. J. M. Anderson, and L. R. Williams, J. Vac. Sci. Technol. B 7, 1203(1989).

10 X.D. Zhu, Ph.D. thesis, Berkeley, unpublished.

11 J. E. Reutt-Robey, D. J. Doren, Y. J. Chabal, and S. B. Christman, Phys. Rev. Lett. 61, 2778(1988).

12 G. Doyen and G. Ertl, Surf. Sci. 43, 197(1974).

${ }^{13}$ R. J. Behm, G. Ertl, and V. Penka, Surf. Sci. 160, 387(1985). 


\section{Figure Captions}

Figure 1: $\mathrm{Ni}(110)$ surface with (a) $\mathrm{c}(4 \times 2)$ and (b) p2gm $2 \times 1$ CO superstructures 011 it. The sizes of $\mathrm{Ni}$ atoms and $\mathrm{CO}$ molecules are not shown in proportion.

Figure 2: Normalized first-order SH diffraction signal versus time at different temperatures for $\mathrm{CO}$ diffusion along (a) [170] , (b) [001] and (c) the direction bisecting [110] and [001] on the Ni(110) surface. The solid lines are the exponential fits with Eq.(9).

Figure 3: Diffusion coefficient $D$ versus reciprocal temperature $1 / T$ in an Arrhenius plot for $\mathrm{CO}$ diffusion on $\mathrm{Ni}(110)$ along [110], [001], and the direction bisecting the two $\left(\phi=45^{\circ}\right)$. The solid lines are least square fits by Eq.(10) and Eq. (11) with $E_{\text {diffl}}[1 \overline{1} 0]=1.1 \mathrm{kcal} / \mathrm{mol}, D_{0}[1 \overline{1} 0]=3.8 \times 10^{-9} \mathrm{~cm}^{2} / \mathrm{sec}$, and $E_{\text {difft }}[001]=3.1 \mathrm{kcal} / \mathrm{mol}, \mathrm{D}_{0}[001]=4.8 \times 10^{-6} \mathrm{~cm}^{2} / \mathrm{sec}$.

Figure 4: Diffusion coefficient $\mathrm{D}$ for $\mathrm{CO} / \mathrm{Ni}(110)$ as a function of azimuthal angle $\phi$ away from [110] at $T \sim 110 \mathrm{~K}$. The solid line is calculated from Eq.(3) using the diffusion parameters deduced from Fig.3.

Figure 5: (a) Coverage profile evolutions simulated with a coverage dependent diffusion coefficient given by Eq.(18) with $d=2.5$. The labeled time are in unit of $\tau=s^{2} / 8 \pi^{2} D(0)$, with $D(0)$ defined in Eq. (18). (b) The simulated SH diffraction signal decay with $D(\theta)$ given by Eq. (18) and $\Delta \chi_{\text {eff }}^{(2)}(\theta)$ given by Eq. (14). The exponential decay curve $\exp (-t / \tau)$ is shown for comparison. 
Figure 6: (a) Simulated diffusion coefficient as a function of reciprocal temperature along [110]. The two lines are from the results that will be presented in Chapter V. Starting from the low temperature end, the first point is resulted from fit to the simulated $\mathrm{SH}$ diffraction signal from $\mathrm{t}=0$ to the time with $80 \%$ of the initial signal left $(d=5)$, the second point to $50 \%(d=4)$, the third point to $40 \%(d=3)$, the forth point to $20 \%(d=2.5)$, the fifth point to $1 \%(d=1.5)$. (b) Same as (a) for $D$ along [001] direction with the first point fit to $85 \%$, second point to $60 \%$, third point to $40 \%$, forth point to $20 \%$, and the fitth point to $1 \%$.

Figure 7: Reflection SH signal $\left|\chi_{\text {eff }}^{(2)}(\theta)\right|^{2}$ as a function of CO coverage for a polarization combination $p($ in) $/ p$ (out) with (a) t're plane of incidence parallel to [110], (b) the plane of incidence parallel to [001: 


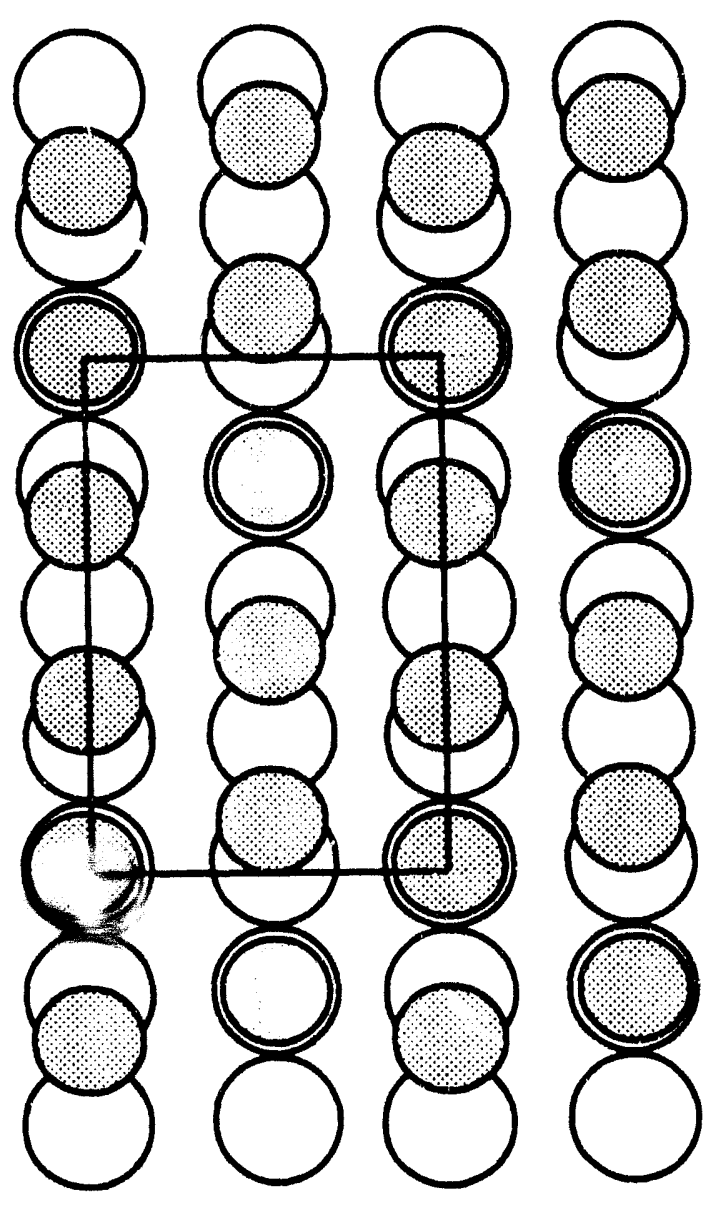

(a)

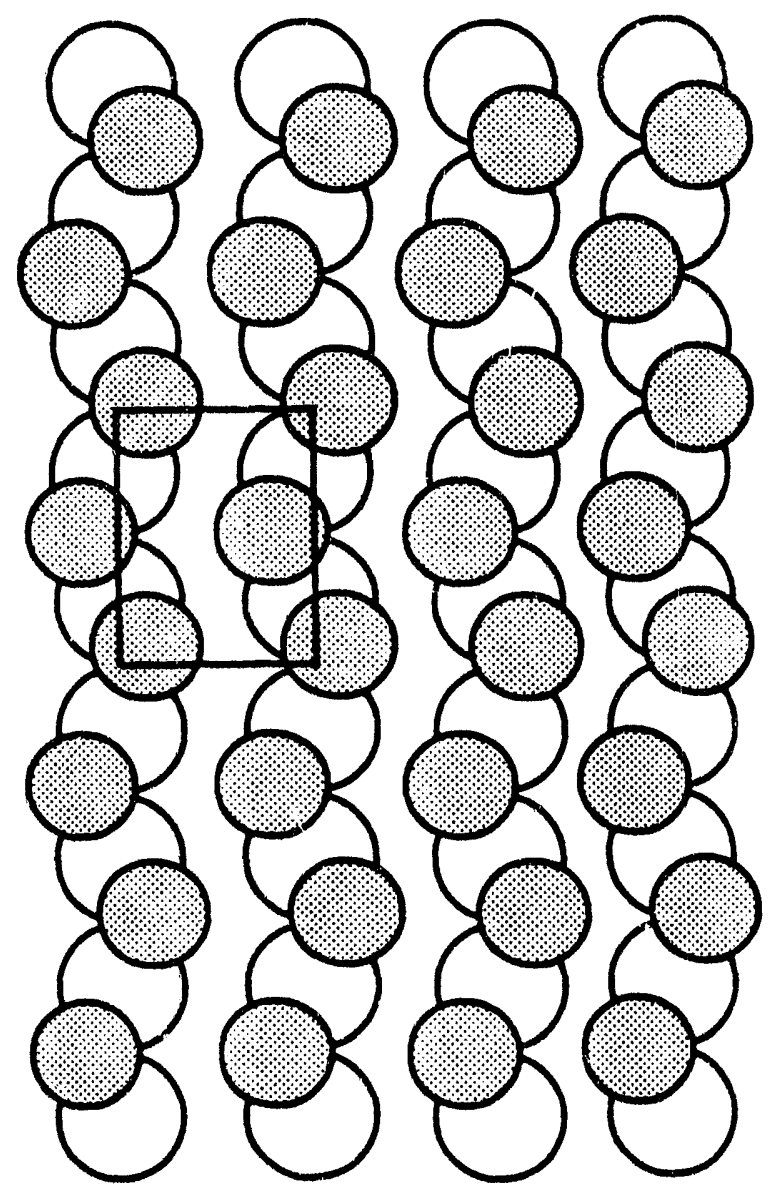

(b)
$\mathrm{Ni}^{\mathrm{N}}$
$\bigcirc$ со

Figure 1 


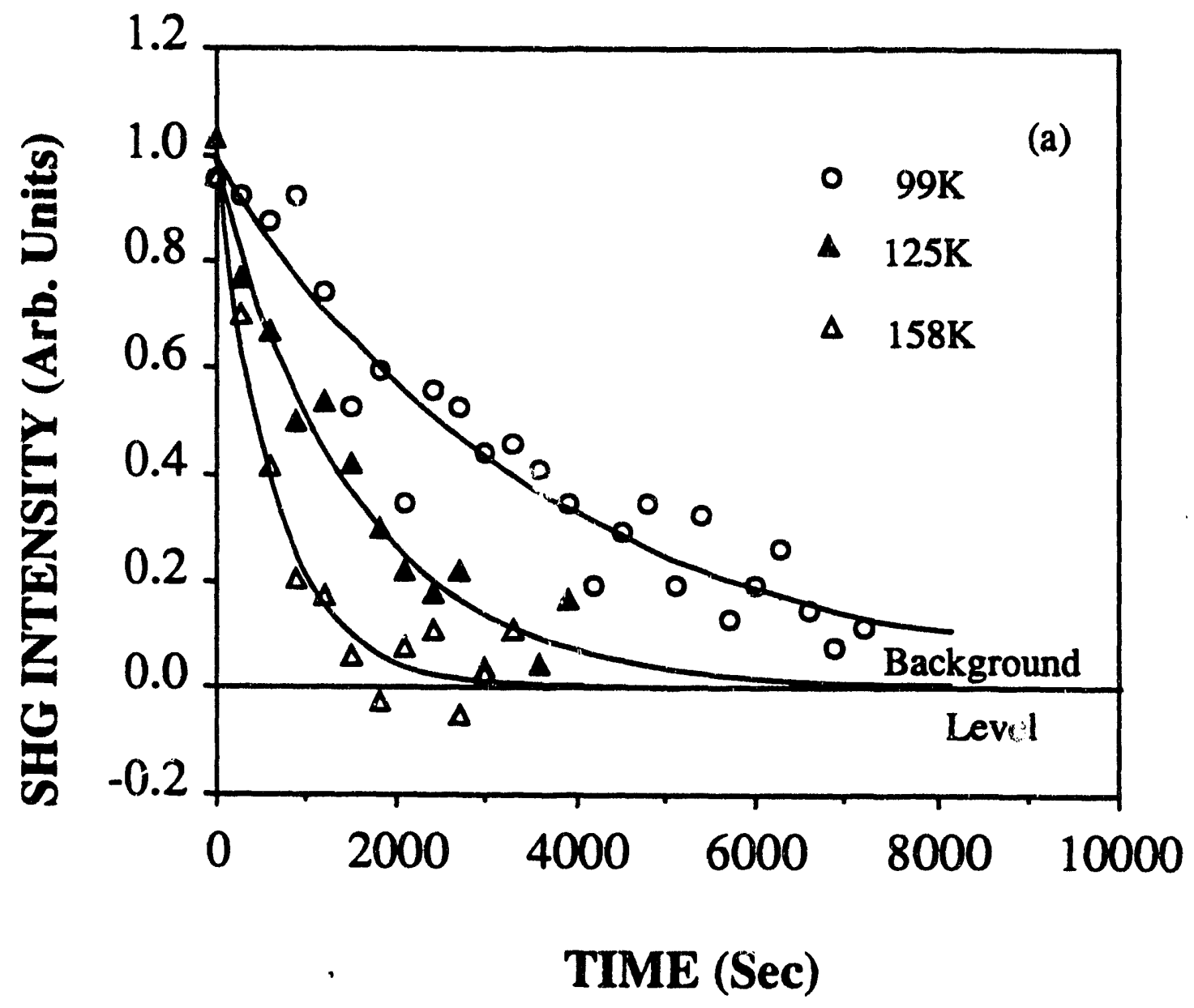

Figure 2(a) 


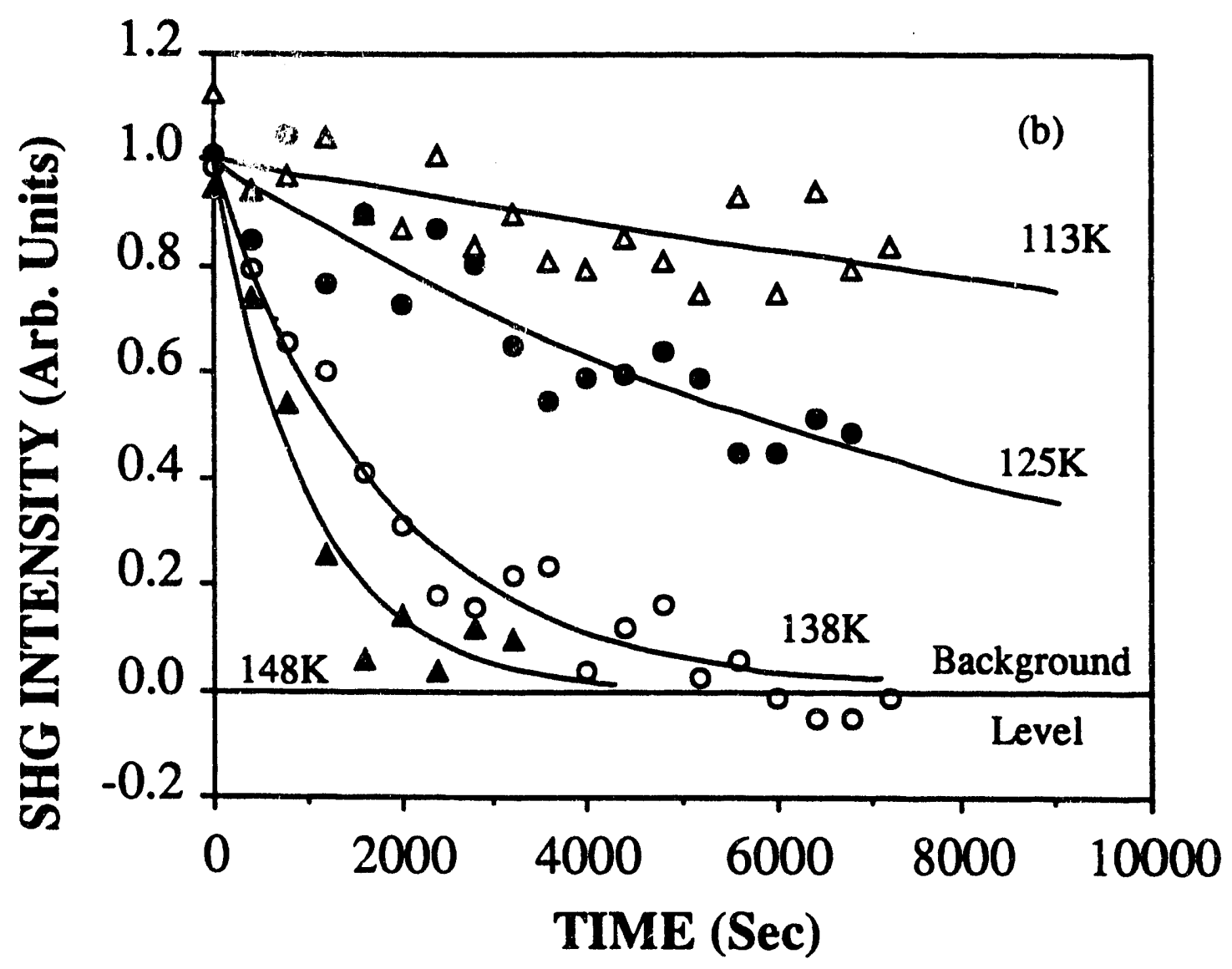

Figure 2(b) 


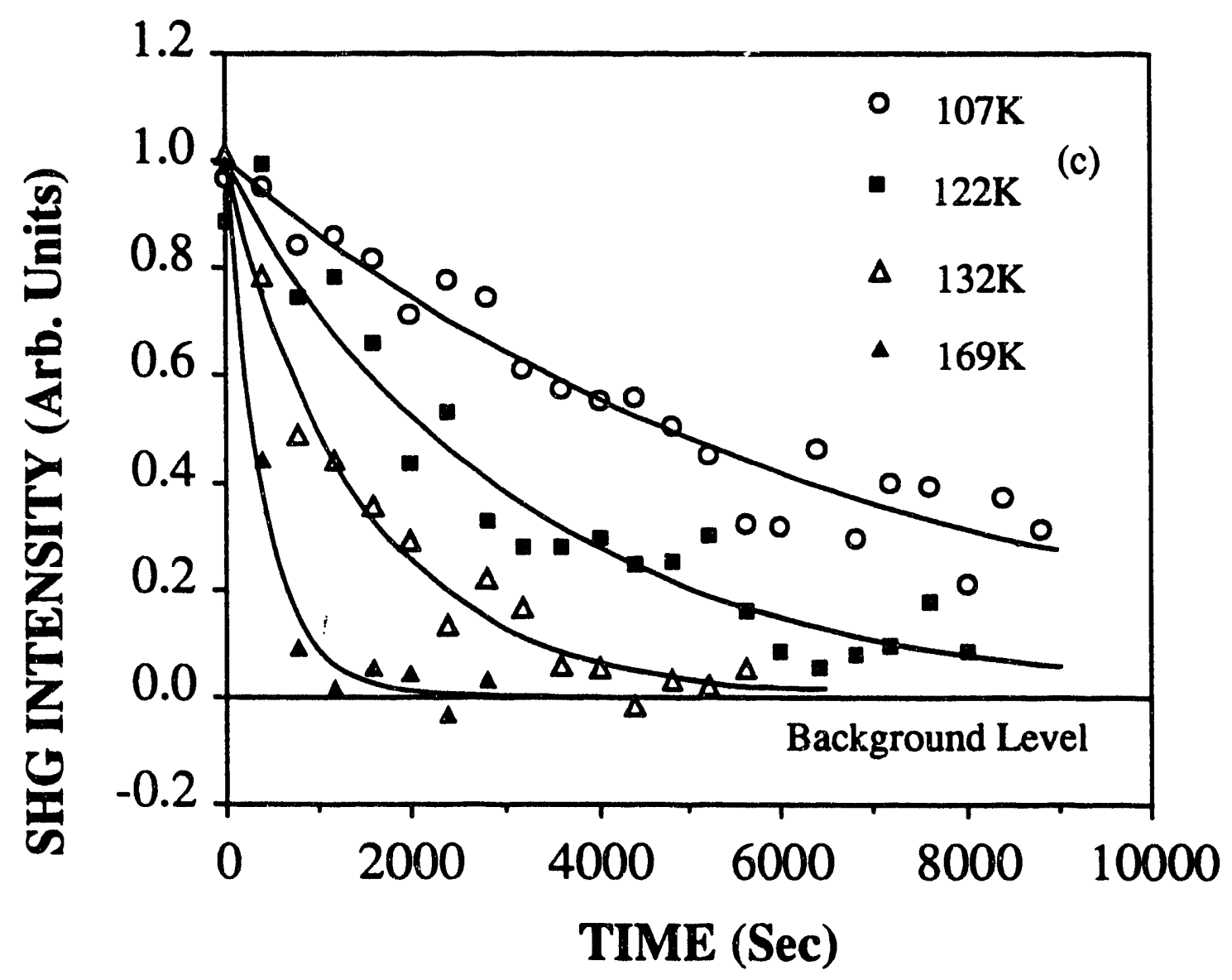

Figure 2(c) 


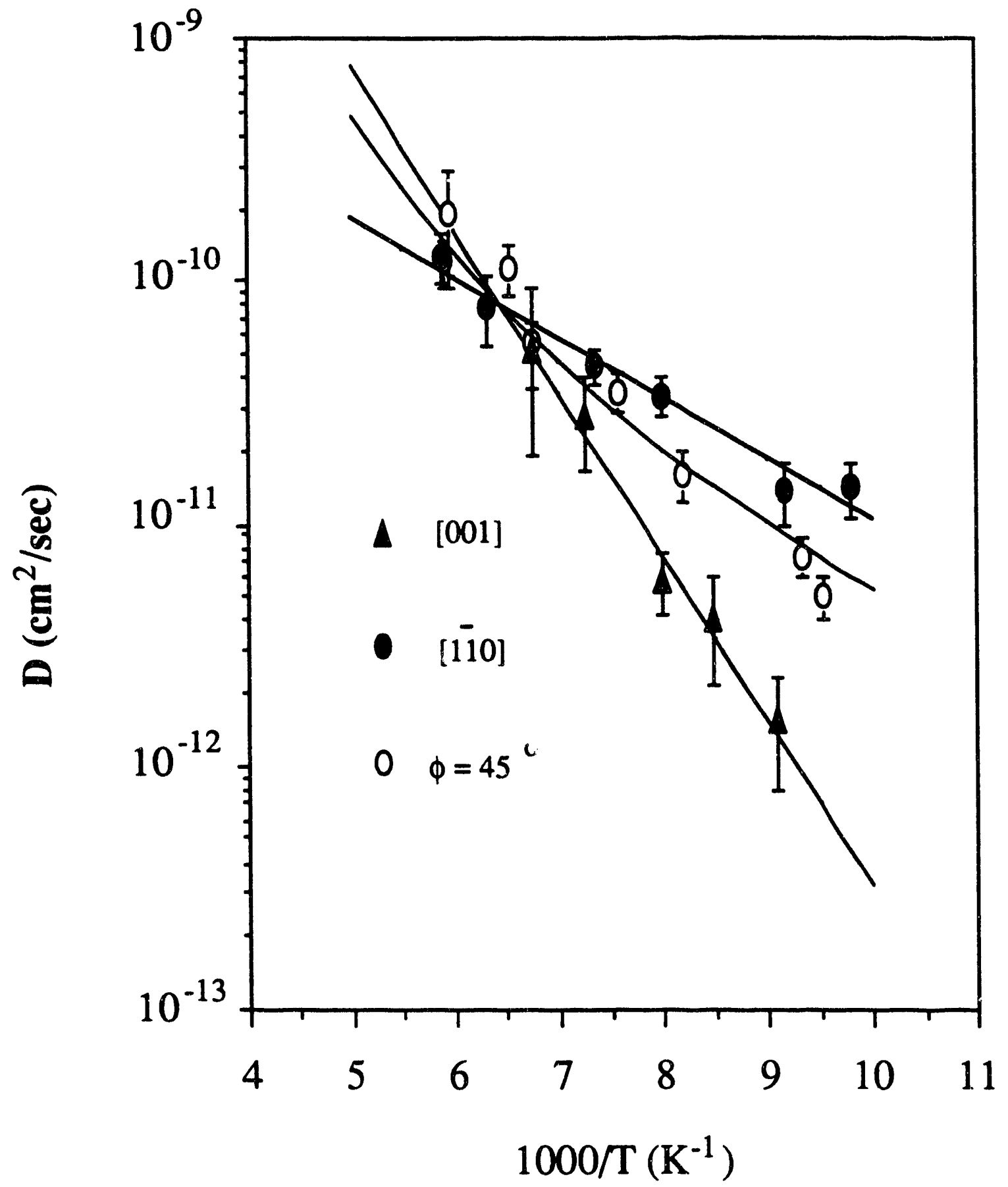

Figure 3 


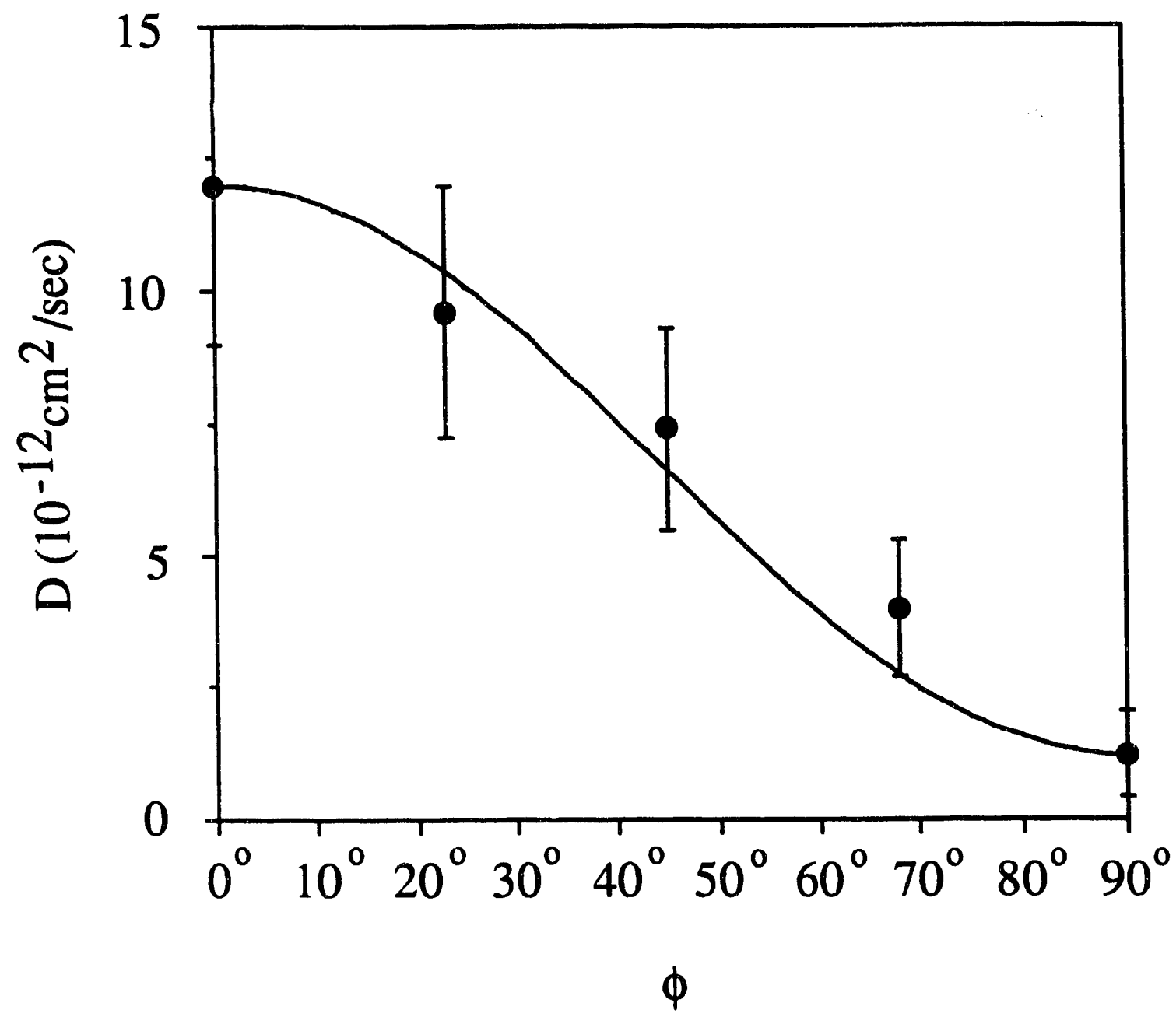

Figure 4 


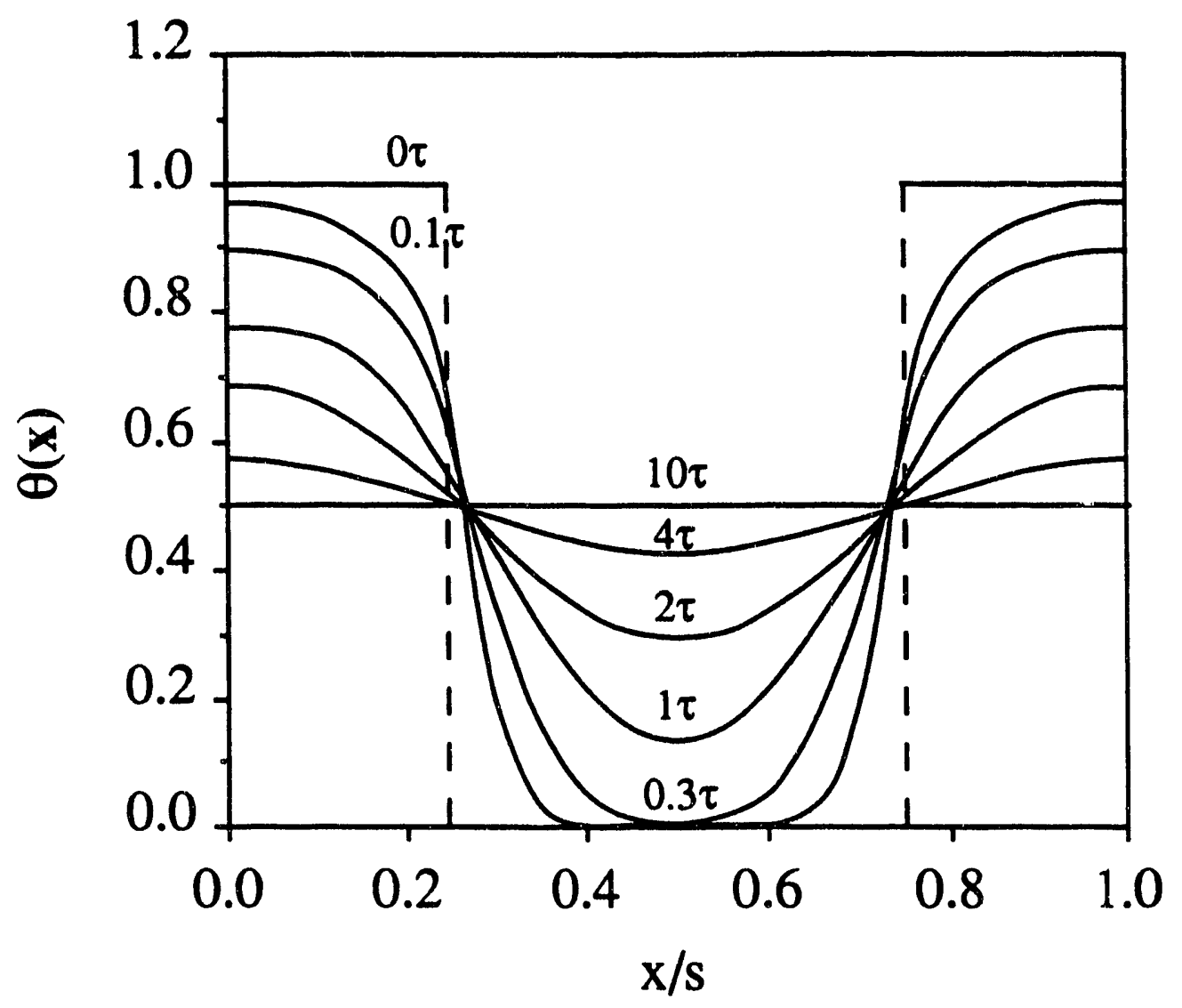

Figure 5(a) 


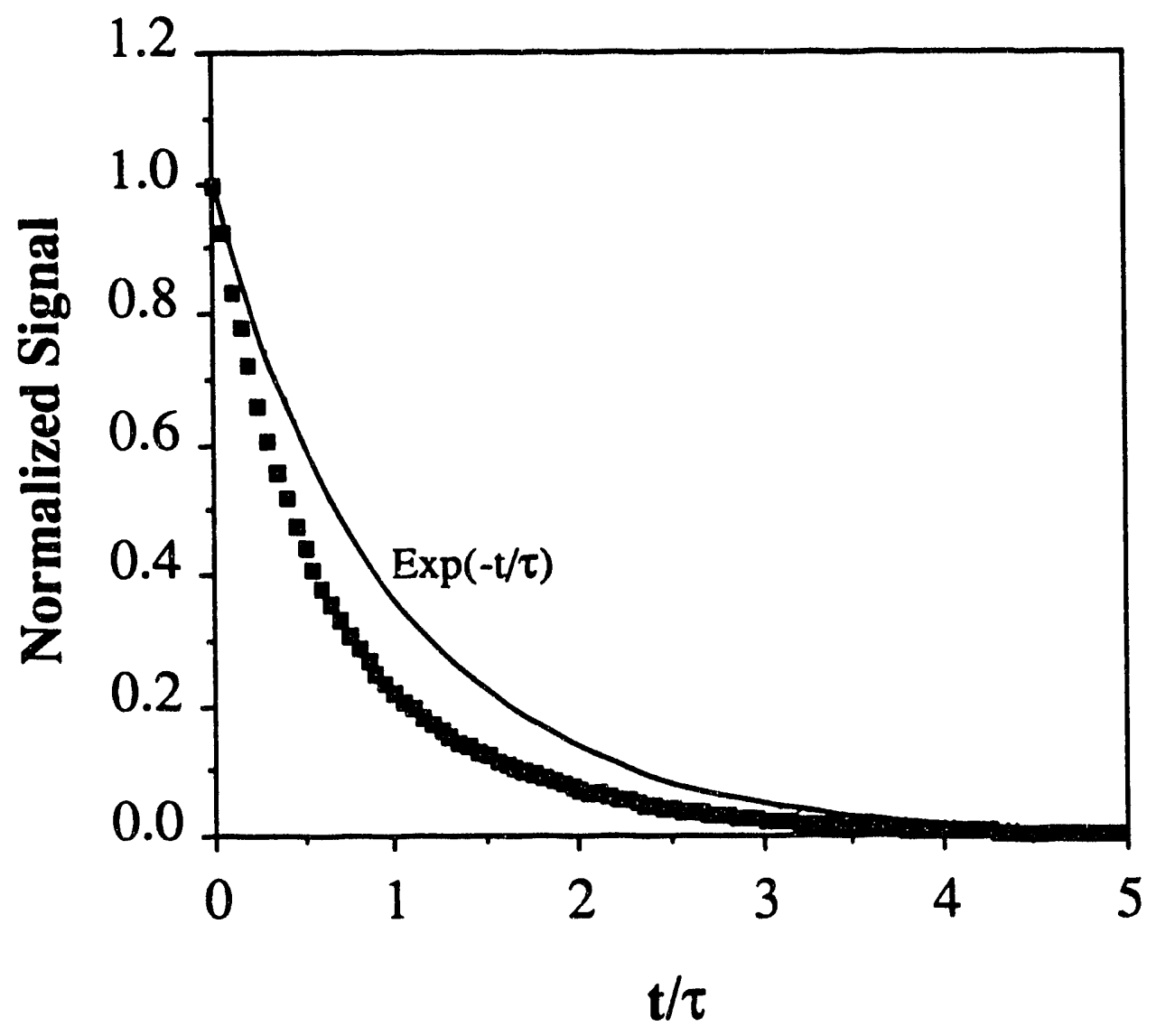

Figure 5(b)

$104 a$ 


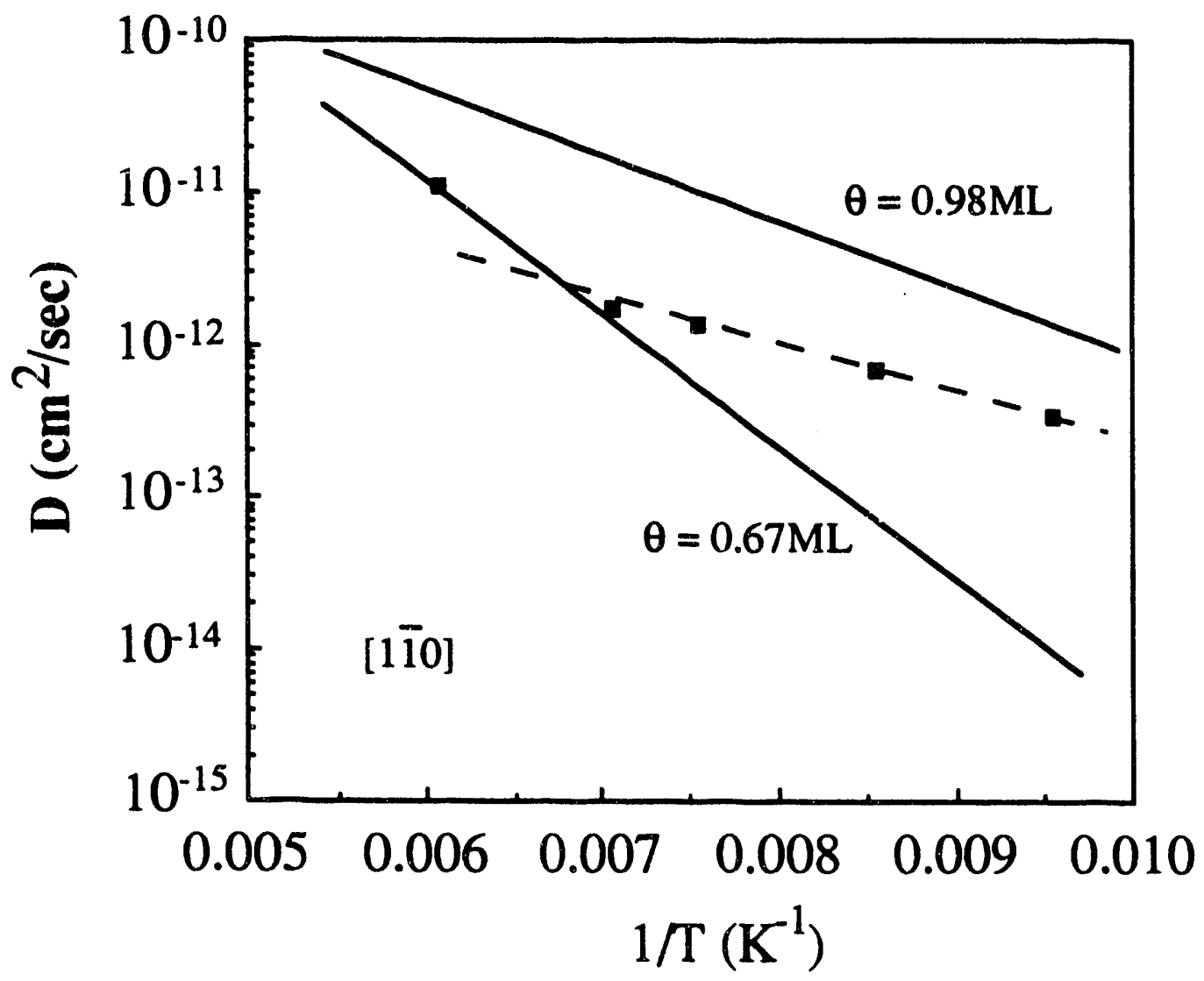

Figure 6(a) 


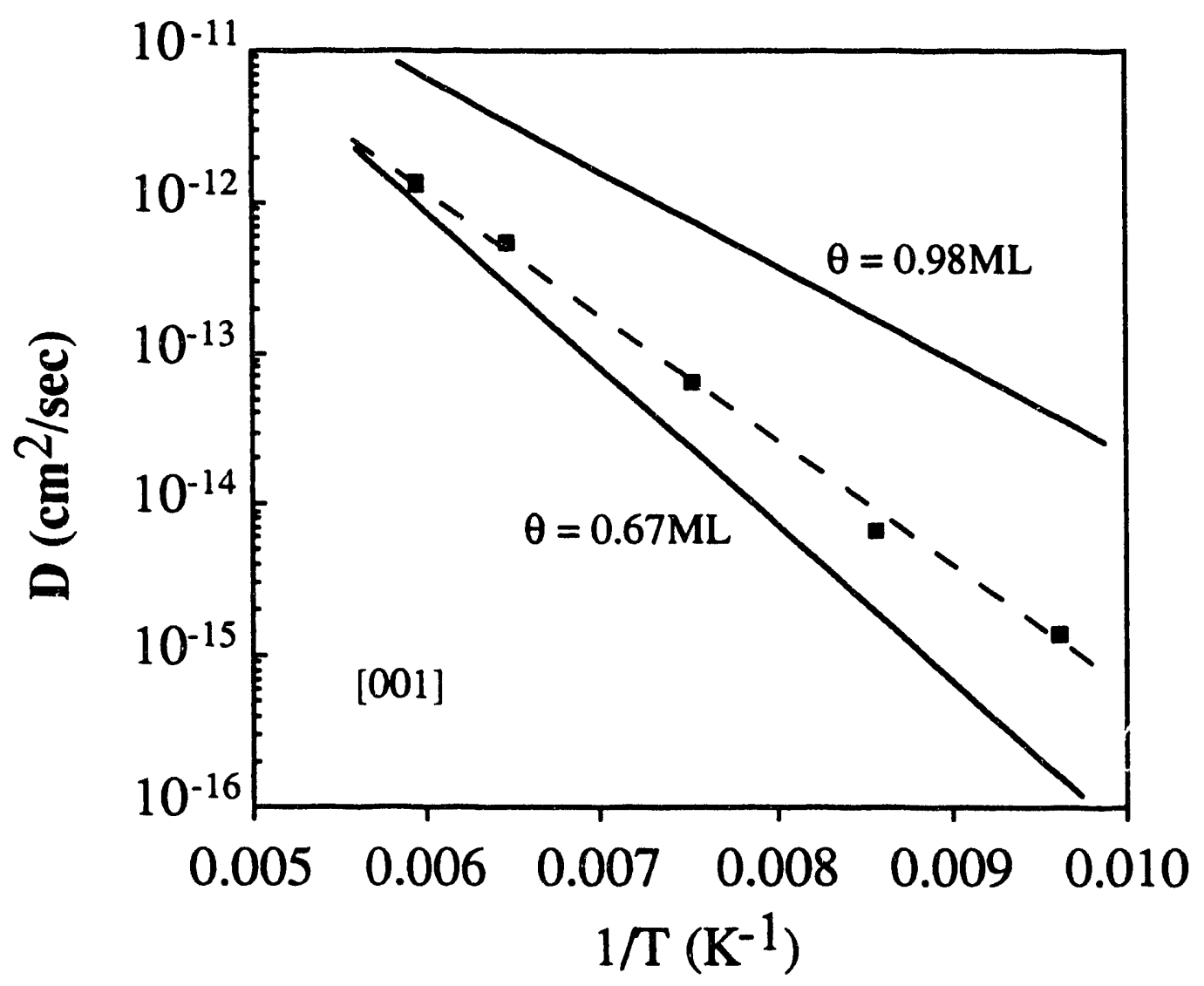

Figure 6(b) 


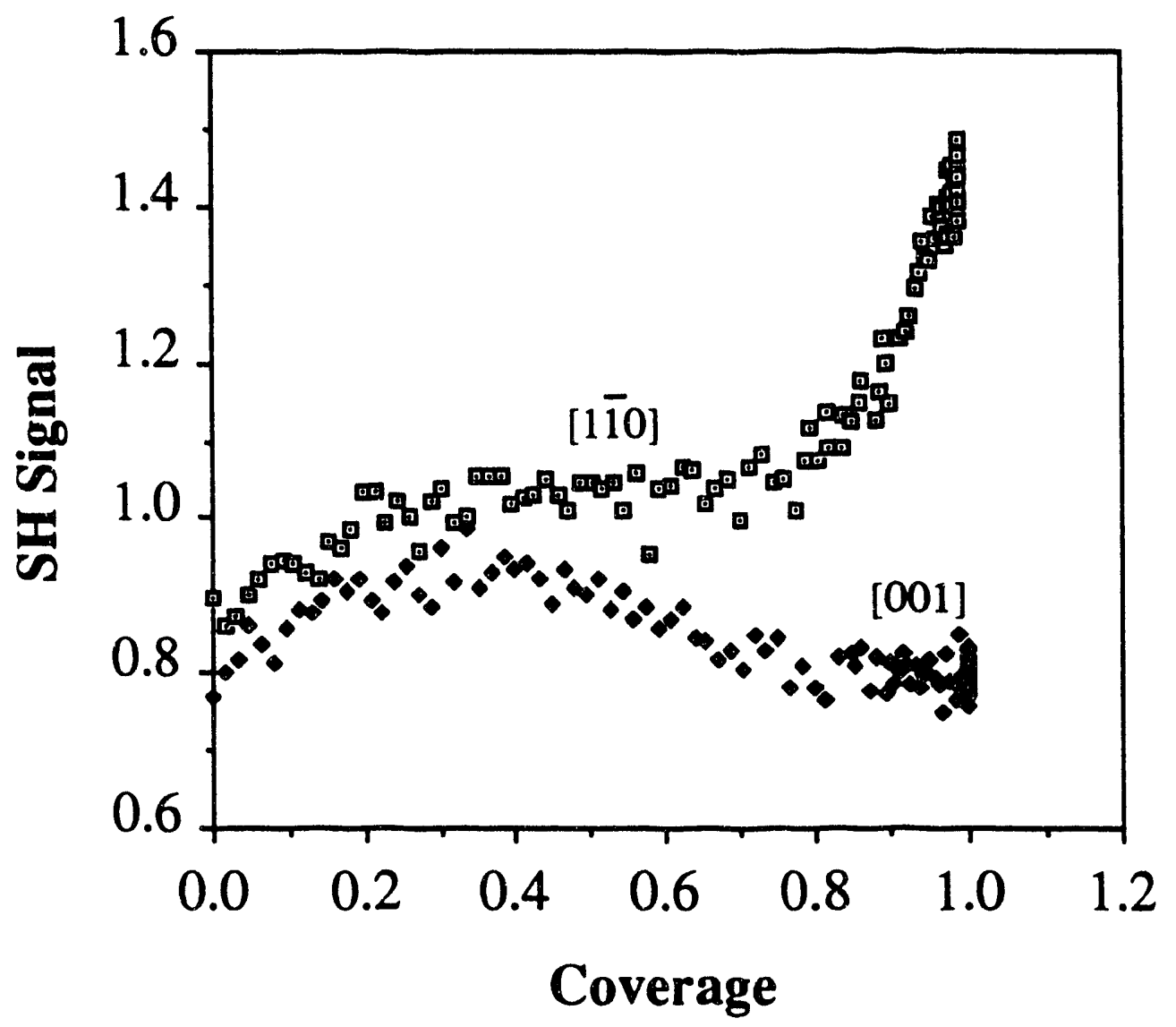

Figure 7 


\section{Surface Diffusion with Adsorbate-Adsorbate Interaction: $\mathrm{CO} / \mathrm{NI}(110)$}

\section{A. Introduction}

Surface diffusion is of great importance to many processes in surface science and have received increasing attention in the past decade ${ }^{1}$. For example, a full understanding chemical reactions on surfaces, surface catalysis and crystal growth, all require detailed information about surface diffusion. Tracer surface diffusion which concerns with a single adparticle motion can provide valuable knowledge about adsorbate-substrate interaction. However, it can not account for the more complicated diffusion processes, in which a large number of adsorbed atoms or molecules are involved. The latter case, known as chemical surface diffusion, connects more closely with the real surface problems. The adsorbate-adsorbate interactions can significantly affect chemical diffusion and need to be carefully investigated ${ }^{2}$.

Theoretical studies on chemical diffusion with different adsorbateadsorbate interactions have been reported in the literature ${ }^{2,3}$. In these studies, the adsorbate-adsorbate interactions are treated in the framework of the lattice gas model, and often only nearest-neighbor and the next nearest neighbor interactions are included. Interactions of an adsorbate at the saddle point (activated complex) with its surrounding adsorbates are sometimes considered as well. However, the origin of the adsorbate-adsorbate interactions has been seldom discussed and the difference of their effect on molecules at adsorption sites and at saddle points has never been systematically examined. Intuitively, the induced potential change by a long range adsorbate-adsorbate interaction 
such as dipole-dipole interaction on an adsorption site and a saddle point may only have slight difference and therefore will not alter the diffusion barrier height much. In strong contrast to this, a short range adsorbate-adsorbate interaction can affect the potential at the adsorption site and the saddle point by significantly different amount and consequently alter the barrier height of diffusion. Experimentally, no direct confirmation of this assertion has been made yet.

In this chapter we will present a coverage dependent study for anisotropic surface diffusion of $\mathrm{CO}$ on $\mathrm{Ni}(110)$ using a newly developed technique, namely, the polarization-modulated linear optical diffraction off a monolayer adsorbate grating ${ }^{4}$. The $\mathrm{CO} / \mathrm{Ni}(110)$ system is interesting because the short range adsorbate-adsorbate interaction occurs only at high coverages $^{5}$. Therefore, the effects of different types of adsorbate-adsorbate interactions on surface diffusion can be studied by varying the CO coverage. Moreover, the $\mathrm{Ni}(110)$ surface is anisotropic and one may wonder if an anisotropy in the coverage dependence of surface diffusion also exists. Our results have unambiguously shown that the diffusion activation energies are influenced by the CO-CO short-range interaction but not by the long-range dipole-dipole and $\mathrm{CO}-\mathrm{Ni}-\mathrm{CO}$ interactions. The anisotropy of the adsorbatesubstrate interaction affecting surface diffusion is present, but the short-range $\mathrm{CO}-\mathrm{CO}$ interaction does not seem to have appreciable effect on the anisotropy of surface diffusion.

\section{B. Experimental Details}

The experiment was performed in an ultrahigh vacuum (UHV) chamber 
with a base pressure of $2.0 \times 10^{-10}$ torr. The single crystal $\mathrm{Ni}(110)$ sample was cut and mechanically polished to within $0.2^{\circ}$ from the (110) plane, and mounted vertically on a rotatable sample holder capable of more than $90^{\circ}$ of rotation about [110]. Before any measurement, the sample was treated by many sputtering and annealing cycles for few days to reduce impurities such as $S, O$, and $C$ in the bulk. A normal cleaning procedure was adopted subsequently to clean the sample surface: it was first $\mathrm{Ar}^{+}$sputtered at $1.0 \times 10^{-4}$ torr $\mathrm{Ar}$ pressure with a $500 \mathrm{~V}$ beam voltage for approximately 30 minutes at room temperature and then annealed at $1120 \mathrm{~K}$ for $10 \mathrm{~min}$ followed by slow cooling at a rate of $\sim 0.5 \mathrm{~K} / \mathrm{sec}$ to $820 \mathrm{~K}$ and a rate of $\sim 2 \mathrm{~K} / \mathrm{sec}$ to room temperature. Auger spectra showed no detectable surface impurities $(<0.3 \% S, O$ and $C)$ after this procedure. A sharp $1 \times 1$ LEED pattern from a clean $\mathrm{Ni}(110)$ surface and a clear $2 \times 1$ LEED pattern from a full CO monolayer on Ni(110) could be observed. They ensured that the surface was well ordered. Liquid nitrogen cooling and electron beam heating were used in the experiment to control the sample temperature. A Chromel-Alumel thermal couple welded to the sample was used to monitor the sample temperature. The temperature could be controlled to within $\pm 1 \mathrm{~K}$.

The diffusion experiment was conducted in the temperature ranges of $140 \mathrm{~K}$ to $220 \mathrm{~K}$ for the $[1 \overline{1} 0]$ direction and $180 \mathrm{~K}$ to $240 \mathrm{~K}$ for the [001] direction for coverages up to $0.8 \mathrm{ML}$. For higher coverages in the [001] direction somewhat lower temperatures were chosen in order to avoid possible $\mathrm{CO}$ adlayer phase transitions ${ }^{6}$. Immediately before each measurement, the sample was flashheated to $600 \mathrm{~K}$ to remove residual adsorbed molecules from the ambient, mostly hydrogen and $\mathrm{CO}$, or previously adsorbed $\mathrm{CO}$ monolayer. The adsorption of $\mathrm{CO}$ on the $\mathrm{Ni}(110)$ surface was carried out at approximately $180 \mathrm{~K}$ by introducing $\mathrm{CO}$ into the chamber through a leak valve. The $\mathrm{CO}$ coverage on 
$\mathrm{Ni}(110)$ at this temperature was calibrated by thermal desorption spectroscopy (TDS). The accuracy in determining the CO coverage was about $0.03 \mathrm{ML}$. We used a polarization-modulated linear optical diffraction technique which has been described in Ref. [4] and Chapter III to measure CO diffusion. The CO adsorbate grating was created by laser-induced thermal desorption with two interfering pulsed laser beams from a single-mode Q-switched Nd:YAG laser (pulse width of $10 \mathrm{~ns}$ at $1.06 \mu \mathrm{m})^{7}$. The grating period was $s \sim 3 \mu \mathrm{m}$. The depth of the adsorbate grating was controlled by adjusting the beam intensities properly. We chose to have a coverage motulation of $~ 0.03 \mathrm{ML}$ on top of an average coverage varying from $0.07 \mathrm{ML}$ to $0.97 \mathrm{ML}$. With such a small coverage modulation, the diffusion coefficient can be very well approximated by a coverage independent constant and the coverage dependence of the diffusion should only come from the average coverage. To probe the adsorbate grating by linear optical diffraction, a 5-mW He-Ne laser was used. The probe beam was polarization-modulated, which was achieved by passing the beam through a photoelastic modulator. First-order diffraction from the grating was selected by an aperture and detected by a lock-in amplifier. The starting signal-to-noise ratio was on the order of 10.

The detail derivation of the relation between the diffraction signal and the diffusion coefficient is referred to Ref. [4] and Chapter III. Here, we only mention that upon diffusion the first order diffraction signal decays exponentially with time and is given by

$$
S_{1}=S_{01} \exp \left(-8 \pi^{2} D t / s^{2}\right)
$$

where the exponent is directly proportional to the diffusion coefficient $D$. In determining $D$, only the decay time constant and the grating spacing s need to 
be known accurately. The detail shape of the grating is of no consequence. To deduce the diffusion activation energy and the preexponential factor from $D$ following the Arrhenius law, measurements at five temperatures or more were made for each coverage. Surface diffusion along different directions were measured by setting the adsorbate grating in the appropriate directions.

\section{Results and Discussion}

\section{a) Measurement Accuracy}

Representative diffraction decay curves are shown in Fig. 1(a) and (b) for average coverage $\theta=0.98$ for diffusion along [1 $1 \overline{1} 0]$ and [001] directions, respectively. The decay time constant deduced from such curves can be usually determined to within $\pm 15 \%$, however, the accuracy of determining the diffusion coefficients in our experiment is rendered to $\pm 50 \%$ by the irreproducibility of the measurements. Two sources for this irreproducibility could exist, one being the intrinsic property to the sample, the other being the laser effect of the desorbing laser beams.

Experimentally, we have explicitly showed the existence of the laser effect by shining a second laser pulse (single beam) onto an adsorbate grating at certain time after its creation. Some typical data are shown in Fig. 2 for initial CO coverage $\theta=0.5 \mathrm{ML}$ at $T=210 \mathrm{~K}$. The decay time constants of the diffraction signal before and after this laser pulse are all indicated along the curves. The accuracy of the deduced time constants is $\sim \pm 5 \%$. It is clear that the decay time constant has been altered by this second laser pulse and becomes longer or shorter in a random fashion. Since the coverage effect has been properly 
avoided by working with initial coverage $\theta=0.5$, where $D(\theta)$ is coverage independent (see subsection b)), the change in the decay time constant should solely come from the laser effect on the substrate surface. The background level change induced by the second laser pulse in curve (a) and (c) further indicated the existence of such effects. However, the nature of this laser effect is not apparent. If the laser pulse only causes surface damage the decay time constant should change in only one direction. Only if the laser can also anneal the surface, the decay time constant can change randomly in both directions.

The above experiment cannot rule out the possibility that the observed irreproducibility could be an intrinsic property of the surface. Methods that do not perturb a surface between diffusion measurements should be applied to address this issue. The Fluctuation-correlation field emission microscopy(FEM) method, which has an accuracy of $\pm 15 \%$ to deduced diffusion coefficient from a single run, seems to be ideal for this purpose. The fact that even with FEM an irreproducibility of $\sim 2$ has been observed indicates that the irreproducibility could be the intrinsic nature of metal surfaces ${ }^{8}$.

\section{b) Coverage Dependent Diffusion Results}

Diffusion coefficients versus reciprocal temperature $1 / T$ for coverage $\theta=0.98$ and 0.48 in both [110] and [001] directions are plotted in Fig. 3 as examples. The solid lines are best fits by the Arrhenius law, 


$$
D=D_{0} \exp \left(-\frac{E_{\text {diff }}}{k_{B} T}\right)
$$

with $D_{0}$ as the preexponential factor, $E_{\text {diff }}$ as the diffusion activation energy, and $k_{B}$ as the Boltzmann constant. The deduced diffusion activation energies and the preexponential factors for $\mathrm{CO}$ diffusion on $\mathrm{Ni}(110)$ surface both along [110] and [001] directions are shown in Fig. 4 (a) and (b). In Fig. 5 the diffusion coefficients as a function of coverage are depicted for three temperatures for $\mathrm{CO}$ diffusion in [001] direction.

The coverage dependence of diffusion are similar in both directions as shown by Fig. 4. The diffusion activation energies are almost constants up to a coverage of $0.7 \mathrm{ML}$ and then drop by $\sim 2 \mathrm{kcal} / \mathrm{mol}$ at full coverage. The preexponential factors follow similar trends. The anisotropy of diffusion can be seen from both Fig. 3 and Fig. 4. The activation energies in the low coverage regime are $4.7 \mathrm{kcal} / \mathrm{mol}$ along [001] and $4.0 \mathrm{kcal} / \mathrm{mol}$ along [110]. The preexponential factors have a difference around a factor of 3 , with the one along [110] larger. In the high coverage range the diffusion activation energies in both directions drop by about the same amount to reduce the activation energy in the [001] direction to $2.8 \mathrm{kcal} / \mathrm{mol}$ and in the [110] direction to $2.0 \mathrm{kcal} / \mathrm{mol}$. This result indicates that the adsorbate-adsorbate interaction has little effect on the anisotropy of the diffusion activation energy.

\section{c) Discussion on Coverage Dependence}

Adsorption sites of $\mathrm{CO}$ on $\mathrm{Ni}(110)$ have been studied with dynamical LEED $^{9}$, electron energy loss spectroscopy (EELS) ${ }^{10}$ and reflection-adsorption 
infrared spectroscopy (RAIRS) ${ }^{11}$. Below $0.7 \mathrm{ML}$ (theoretically, it should be $0.67 \mathrm{ML}$ ), CO adsorbs on both short-bridge and top sites along the $\mathrm{Ni}$ atomic rows with a coverage independent occupation ratio ${ }^{11,12}$. The separation between the $C O$ molecules is at least $3.74 \AA$ in the [110] direction. Certain superstructures such as $c(8 \times 2), c(4 \times 2)$ have been observed by some authors ${ }^{6,9,13}$ and these structures indicate that the site registry is not ideal ${ }^{13}$. Above this coverage the CO molecules are pushed to short-bridge sites ${ }^{9,13}$ (EELS results prefer top sites ${ }^{10}$ ) and tilt in the [001] and [001] directions with a tilt angle of $19^{\circ}$ with respect to the surface norma $\left.\right|^{5,6}$. At the full coverage a p2mg(2x1) structure is formed ${ }^{5,6}$. The adsorbate-adsorbate interactions have been investigated by TDS $^{14}$, EELS ${ }^{10}$, angular resolved photoemission spectroscopy (ARUPS) ${ }^{15}$ and other techniques. While ARUPS concerns more about the electronic states, TDS and EELS do provide binding energy information. It has been found by TDS that an $\alpha_{2}$ desorption state with a low desorption energy appears at coverage above $0.7 \mathrm{ML}$, with some dispute in the exact uptake coverage ${ }^{13,14,16}$. The coverage dependent desorption energy ${ }^{14}$ is depicted in Fig. 4(a) along with the diffusion activation energies. The correlation between them is excellent; both the diffusion activation energies and the desorption energy show a decrease above coverage $0.7 \mathrm{ML}$. The drop in the desorption energy is about $4 \mathrm{kcal} / \mathrm{mol}$ and in the diffusion activation energies is about $2 \mathrm{kcal} / \mathrm{mol}$. Up to this point, we can conclude that the long range dipoledipole interaction and $\mathrm{CO}-\mathrm{Ni}-\mathrm{CO}$ indirect interaction, which are present at all coverages, do not influence the diffusion activation energy and the desorption energy. This is because first they are of long range. The potential at the saddle points may have been affected by the same amount as that at the adsorption site. Second, the dipole-dipole interaction is repulsive and the CO-Ni-CO interaction is attractive ${ }^{17}$. Their effects on the surface potential may get 
canceled to certain extent. Above $0.7 \mathrm{ML}$ coverage, since the $\mathrm{CO}$ molecule has a diameter of $2.8 \AA^{6}$, and the Ni atom has a diameter of $2.49 \AA$, the CO-CO molecules start to have significant orbital overlapping in the [110] direction (The nearest-neighbor CO's in the [001] direction do not have this interaction because the distance between two $\mathrm{Ni}$ rows is $3.52 \AA$, larger than the diameter of $\mathrm{CO}$ molecules. The displacement of the $\mathrm{CO}$ molecules makes the situation somewhat more complicated but will not change this result, see Fig. 6). The orbital overlapping is significantly reduced once a $\mathrm{CO}$ molecule jumps to a saddle point, which is at least $\sim 3.10 \AA$ in the $[1 \overline{1} 0]$ direction and $\sim 2.72 \AA$ in the [001] direction away from the nearest-neighbor $\mathrm{CO}$ molecules ${ }^{9}$. Because of this repulsive CO-CO interaction, a net reduction of diffusion activation energy can result. The same reason should be responsible for the drop in the desorption energy.

\section{d) Comparison with Other Nickel Surfaces}

A comparison with $\mathrm{CO}$ diffusion on the other two low Miller index planes of nickel, namely $\mathrm{Ni}(111)$ and (100), is worthwhile. CO diffusion on $\mathrm{Ni}(111)$ has been studied in detail by FEM method and no coverage dependence for the activation energy $(6.8 \mathrm{kcal} / \mathrm{mol})$ has been found ${ }^{18}$. Even at the saturation coverage of $0.57 \mathrm{ML}$ for $\mathrm{a}(\sqrt{7} / 2 \times \sqrt{7} / 2) \mathrm{R} 19.1^{0}$ superstructure $^{19}$ for $\mathrm{CO} / \mathrm{Ni}(111)$, the shortest distance between $\mathrm{CO}-\mathrm{CO}$ is about $3.30 \AA$, which is still much larger than the $\mathrm{CO}$ diameter. Therefore, no $\mathrm{CO}-\mathrm{CO}$ direct interaction is expected on this surface. The diffusion results of $\mathrm{CO} / \mathrm{Ni}(111)$ then confirm our findings from $\mathrm{CO} / \mathrm{Ni}(110)$ that the long range interactions do not affect the diffusion activation energy. However, this agreement is contrasted by a strong coverage dependent desorption energy of $\mathrm{CO} / \mathrm{Ni}(111)$, which is $30 \mathrm{kcal} / \mathrm{mol}$ up to $0.35 \mathrm{ML}$ 
and drops monotonically to $14.5 \mathrm{kcal} / \mathrm{mol}$ to the saturation coverage $\mathrm{e}^{20}$. The difference in the coverage dependences between the desorption energy and the diffusion activation energy can only be explained by assuming that the long range interactions affect them in different ways. The situation for $\mathrm{CO} / \mathrm{Ni}(100)$ is different. CO diffusion has been measured for only a few coverages by the LID method $^{21}$ and a coverage dependent activation energy has been found with $E_{\text {diff }}=6.4 \mathrm{kcal} / \mathrm{mol}$ at $\theta=0.25 \mathrm{ML}$, and $E_{\text {diff }}=4.6 \mathrm{kcal} / \mathrm{mol}$ at $\theta=0.4$ and

$0.64 \mathrm{ML}$ (saturation coverage). While it is clear that the CO-CO overlapping interaction is not responsible for this drop since the CO-CO separation at saturation is at least $5.0 \AA^{22}$, the coverage dependence has been interpreted as due to the CO superstructures $c(2 \times 2)$ and $c(2 \sqrt{2} \times \sqrt{2}) R 45^{\circ}$ at the two high coverages $^{21}$. No reliable desorption energy data exists for this system. However, qualitatively, the desorption energy has been estimated to also have a coverage dependence, namely $26 \mathrm{kcal} / \mathrm{mol}$ at low coverage and $10 \mathrm{kcal} / \mathrm{mol}$ at high coverage ${ }^{23}$.

From the data discussed above, we obtain the ratios of diffusionactivation-energy/desorption-activation-energy as: $\geq 0.23$ for $\mathrm{CO} / \mathrm{Ni}(111), \geq 0.24$ for $\mathrm{CO} / \mathrm{Ni}(100)$, and $\leq 0.15$ for $\mathrm{CO} / \mathrm{Ni}(110)$. From these ratios and the absolute diffusion activation energies, $\mathrm{Ni}(110)$ is seen to be the smoothest for $\mathrm{CO}$ diffusion. The fundamental reason for this may have to do with the relaxation of the clean Ni surfaces. It has been found that the first atomic layers of these three surfaces contract by different amount: about $1 \%$ for $\mathrm{Ni}(111)^{24}$ and $\mathrm{Ni}(100)^{25}$, and $9 \%$ for $\mathrm{Ni}(110)^{26}$. The significantly large contraction in $\mathrm{Ni}(110)$ can result into a much smoother electronic charge density and thus a smoother surface potential corrugation ${ }^{27}$. 


\section{e) Discussion on Anisotropic Activation Energy}

Diffusion activation energies along [1 $1 \overline{1} 0]$ and [001] directions of $\mathrm{Ni}(110)$ are expected to be different. Due to the relatively smooth electronic charge density along [110] direction, the potential seen by a $\mathrm{CO}$ molecule along that direction should be less corrugated than that along [001] direction. A theoretical calculation by Doyen and Entl indeed predicted a surface potential variation of $\sim 1.25 \mathrm{kcal} / \mathrm{mol}$ along [1 $1 \overline{1} 0]$ and $\sim 2.25 \mathrm{kcal} / \mathrm{mol}$ along the [001] direction ${ }^{28}$. The experimental observation of streak-like $c(4 \times 2)$ and $c(8 \times 2)$ LEED patterns by Behm et al, which was interpreted as a consequence of $\mathrm{CO}$ occupation at intermediate positions other than the high symmetry sites (on-top and shortbridge sites) along the [1피 direction, further indicated that the potential corrugation along $[1 \overline{1} 0]$ is smoother than that along $[001]^{13}$. The measured diffusion activation energies of $\mathrm{CO}$ on $\mathrm{Ni}(110)$ for all coverages unequivocally showed this predicted anisotropy. However, our observed $\sim 20 \%$ anisotropy of the $\mathrm{CO}-\mathrm{Ni}$ interaction appearing in the diffusion activation energies is somewhat insignificant. This weak anisotropy is in good agreement with the EELS measurement ${ }^{10}$, in which the vibrational frequencies of the two CO frustrated translational modes (corresponding to the two diffusion coordinates here) also showed very little anisotropy. In the case of adatom diffusion of $\mathrm{Ni} / \mathrm{Ni}(110)$, the anisotropy of diffusion activation energies measured by $\mathrm{FIM}$ is 40\%, again a relatively small value.

We can further seek for a quantitative agreement between the diffusion results and the EELS results. The $\mathrm{CO}$ frustrated vibrational frequencies spectra of a full $\mathrm{CO}$ monolayer can be estimated from the measured diffusion activation energies with a simple chain model. Considering the force on $\mathrm{CO}$ at a Ni site 
to be $-M \omega_{0}^{2} x$ and the force from a nearest $C O$ to be proportional to the difference of their displacements, the equation of motion for the s-th $\mathrm{CO}$ molecule is then

$$
M \frac{d^{2} x_{s}}{d t^{2}}=-M \omega_{0}^{2} x_{s}-C\left(x_{s+1}+x_{s-1}-2 x_{s}\right)
$$

where $x_{s}$ is the displacement of the s-th $\mathrm{CO}$ molecules, $M$ the mass of $\mathrm{CO}$ molecule, and $\mathrm{C}$ the force constant between $\mathrm{CO}$ molecules(repulsive). Using the standard procedure in textbooks ${ }^{29}$, we can obtain a dispersion relation for the phonon spectrum as

$$
\omega^{2}=\omega_{0}^{2}-\frac{2 C}{M}(1-\cos K a)
$$

where $\mathrm{K}$ is the wave-vector and a the lattice constant. Approximating the force constant by $M \omega_{0}^{2}=E_{a} / a^{2}$ and $C=\varepsilon / a^{2}$, with $E_{a}$ as the diffusion barrier height at zero coverage and $\varepsilon$ as the CO-CO interaction energy, we have obtained downward dispersion relations of phonon spectra of $105 \mathrm{~cm}^{-1}$ to $74 \mathrm{~cm}^{-1}$ from the $\bar{\Gamma}$ point $(K=0)$ to the zone boundary $(K=\pi / 2 a)$ in the [1 $\overline{10}$ ] direction and $80 \mathrm{~cm}^{-1}$ to $62 \mathrm{~cm}^{-1}$ in the $[001]$ direction. Despite the crudeness of the calculation, quantitatively these results are in good agreement with the EELS measurement ${ }^{10}$.

Opposite to what we might expect, the CO-CO adsorbate-adsorbate interaction does not show any anisotropic effect in the diffusion activation energies. The reductions of these diffusion activation energies due to the short range CO-CO direct interaction in both [1T0] and [001] directions are about the 
same, $2.0 \mathrm{kcal} / \mathrm{mol}$. Although we cannot rule out the possibility that the $\mathrm{CO}-\mathrm{CO}$ direct interaction is isotropic, the model we will construct next seems to be more reasonable to explain this absence of anisotropy.

In Fig. 6 (a) and (b), we have shown the adsorbates configurations near the saturation coverage (only one site is not occupied) for $\mathrm{CO}$ diffusion along [110] and [001], respectively. Since only neighboring CO molecules on the same Ni row interact with each other (see subsection c)), a diffusing $\mathrm{CO}$ molecule jumping from its adsorption site to neighboring empty site along [110] can interact with only one nearest neighbor $\mathrm{CO}$. On the other hand, a diffusing $\mathrm{CO}$ along [001] can interact with two nearest $\mathrm{CO}$ molecules. This would lead to anisotropy due to adsorbate-adsorbate interaction on diffusion activation energy if the effect from adsorbate-adsorbate interaction on the saddle points did not cancel it. However, a CO molecule at the saddle point $C$ along [110] is at least $3.10 \AA$ away from all the other $C O$ molecules and thus should not be affected by the direct $\mathrm{CO}-\mathrm{CO}$ interaction. The situation for $\mathrm{CO}$ at the saddle point $C^{\prime}$ along [001] is very different. The distance of $\mathrm{CO}$ at $C^{\prime}$ from the two nearest neighbor $\mathrm{CO}$ molecules is $\sim 2.72 \AA$ without considering possible displacement of the activated complex along the surface normal direction. Therefore, the adsorbate-adsorbate interaction cannot be neglected. It could have compensated the effects on $\mathrm{CO}$ at the adsorption site and resulted in apparently weak anisotropy in the change of diffusion activation energy due to CO-CO interaction.

\section{f) Discussion on the Anisotropy of Preexponential Factor}

From Fig. 4(b), the anisotropy in the diffusion preexponential factors are obvious. However, the usual compensation law is not obeyed. With the diffusion 
activation energies along [001] being larger than those along [1ํㅣ], the associated preexponential factors in the [001] direction are smaller by a factor of 3 than those in the $[1 \overline{1} 0]$ direction. Due to the small difference in the activation energies, this violation of the compensation law may not be very severe.

Transition state theory (TST) can be applied to calculate anisotropy of the preexponential factor once a surface potential is known. Because of the coverage independence of the diffusion coefficient in the low coverage region, we can simplify the calculation by applying the TST in the $\theta->0$ limit (tracer diffusion). The expression for the tracer diffusion coefficient is ${ }^{1}$

$$
D=\frac{1}{4}\left\langle\left.\right|^{2}\right\rangle v=1 / 4\left\langle\left.\right|^{2}\right\rangle v_{0} \exp \left(-E_{a} / k_{B} T\right)
$$

where $l$ is the hopping length, $v$ the hopping frequency, $v_{0}$ the trial frequency and $E_{a}$ the diffusion activation energy. The pre-exponential factor is defined in terms of hopping length I and trial frequency $v_{0}$ by

$$
D_{0}=1 / 4\left\langle\left.\right|^{2}>v_{0}\right.
$$

In the transition state theory, the trial frequency is given by ${ }^{30}$

$$
v_{0}=\frac{k T}{h} \frac{Z_{C}^{\neq}}{Z_{A}},
$$

where $Z_{C}^{\neq}$is the partition function of the adsorbate (excluding the diffusion coordinate) at the saddle point $C$ and $Z_{A}$ is the total partition function of the 
adsorbate in the well A (see Fig.7). In order to calculate the partition functions, we use a surface potential

$$
V(x, y)=E_{\text {diff }}[1 \overline{1} 0]\left(1-\cos \frac{2 \pi x}{a}\right)+E_{\text {diff }}[001]\left(1-\cos \frac{\sqrt{2} \pi y}{a}\right),
$$

where $E_{\text {diff }}[1 \overline{1} 0]$ and $E_{\text {diff }}[001]$ are the measured diffusion activation energies in the two principal directions respectively. The periodicity of the surface potential has been chosen to be the same as that of the substrate, namely a along [1 $1 \overline{1} 0]$ ( $\hat{x}$ direction) and $\sqrt{2} a$ along [001] ( $\hat{y}$ direction). This potential can give correct barrier heights for $\mathrm{CO}$ diffusion. Since the well site $A$ is common, the partition function $Z_{A}$ is the same for both [1 $1 \overline{10}$ ] and [001] directions. The anisotropy in the trial frequencies comes solely from the partition functions at the two different saddle points along the two corresponding directions. The relevant partition functions at the saddle points $C$ and $C^{\prime}$ in [1 $\left.1 \overline{1} 0\right]$ and [001] directions (see Fig. 7) can be written approximately as

$$
\begin{aligned}
& Z_{C}^{\neq}[1 \overline{1} 0]=Z_{C, y v i b .} Z_{C, \text { bending }} Z_{C, z v i b .} Z_{C, \text { in. }} Z_{C, e l}, \\
& Z_{C^{\prime}}^{\ddagger}[001]=Z_{C^{\prime}, x v i b .} Z_{C^{\prime}, \text { bending }} Z_{C^{\prime}, z v i b}{ }{ }_{C^{\prime}, \text { in. }} Z_{C^{\prime}, e l},
\end{aligned}
$$

if the coordinates involved can be separated. Here, $\hat{z}$ is the [110] (surface normal) direction. The electronic, $C O$ internal vibrational, and the $z$-direction vibrational partition functions $Z_{C, e l}\left(Z_{C^{\prime}, \text { el }}\right), Z_{C, \text { in. }}\left(Z_{C^{\prime}, \text { in. }}\right)$, and $Z_{C, z v i b .}\left(Z_{C^{\prime}, z v i b .}\right)$ at the two saddle points certainly would not differ very much because the corresponding potentials seen by the electrons of the molecule or by the molecule as a whole do not have large differences ${ }^{31}$. The anisotropy in the trial 
frequencies should be dominated by the difference in the frustrated translational

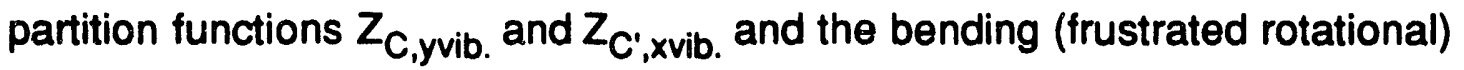
partition functions $Z_{C \text {,bending }}$ and $Z_{C^{\prime} \text {, bending. }}$

The frustrated translational partition functions can be readily calculated by using the surface potential of Eq. (8). Including contribution from the continuum states to the partition functions, we obtain

$$
\begin{aligned}
Z_{C, y v i b} & =\sum_{n=0}^{23} \exp \left(-\frac{n \hbar \omega_{y}}{k_{B} T}\right)+\sum_{k} \exp \left(-\frac{\hbar^{2} k^{2}}{2 M k_{B} T}\right) \exp \left(-\frac{E_{\text {diff }}^{[001]}}{k_{B} T}\right) \\
& =\sum_{n=0}^{23} \exp \left(-\frac{n \hbar \omega_{y}}{k_{B} T}\right)+\frac{\sqrt{2 a}}{\hbar} \sqrt{\frac{M k_{B} T}{2 \pi}} \exp \left(-\frac{E_{\text {diff }}}{k_{B} T}\right), \\
Z_{C^{\prime}, x v i b .} & =\sum_{n=0}^{15} \exp \left(-\frac{n \hbar \omega_{x}}{k_{B} T}\right)+\sum_{k} \exp \left(-\frac{\hbar k^{2}}{2 M k_{B} T}\right) \exp \left(-\frac{E_{d i f f}[1 \overline{1} 0]}{k_{B} T}\right) \\
& =\sum_{n=0}^{15} \exp \left(-\frac{n \hbar \omega_{x}}{k_{B} T}\right)+\frac{a}{\hbar} \sqrt{\frac{M k_{B} T}{2 \pi}} \exp \left(-\frac{E_{\text {diff }}[1 \overline{1} 0]}{k_{B} T}\right),
\end{aligned}
$$

where the vibrational frequencies can be calculated from small vibration formulas: $\omega_{x}=(2 \pi / a)\left[E_{d i f f l}[10] / M\right]^{1 / 2} \sim 2 \pi \times 105 \mathrm{~cm}^{-1}$ for vibration in $\hat{x}$ and $\omega_{y}=$ $\left(2 \pi / \sqrt{2}\right.$ a) $\left[E_{\text {diff }}[001] / M\right]^{1 / 2} \sim 2 \pi \times 80 \mathrm{~cm}^{-1}$ for vibration in $\hat{y}$. The number of discrete states in the summation are truncated by the maximum potential and no anharmonic effect has been included. It turns out that the contribution from the continuum are negligible. The results of the two partition functions are $\mathrm{Z}_{\mathrm{C} \text {,yvib. }}{ }^{\sim 2.45 \text { and } \mathrm{Z}_{\mathrm{C}^{\prime}, \mathrm{xvib}}}{ }^{\sim 2.00}$ respectively. From the above discussion, the 
ratio of the two preexponential factors is given by

$$
\frac{D_{0}[1 \overline{1} 0]}{D_{0}[001]}=\frac{z_{C}^{\neq}[1 \overline{10}]}{Z_{C^{\prime}}^{\neq}[001]} \frac{a^{2}}{(\sqrt{2} a)^{2}} \sim 0.6 \frac{z_{C \text { bending }}}{Z_{C^{\prime} \text { bending }}}
$$

The bending partition functions are somewhat difficult to calculate because the corresponding potentials are not known. Assuming they are not very different at the two saddle points, we then obtain comparable preexponential factors for the two directions. This can be compared with the experimental value of 3 . Considering the approximations made in this estimate, the agreement with the experiment is reasonable.

\section{e) Theoretical Fittings of $D(\theta)$}

The adsorbate-adsorbate interaction effect on surface diffusion has been investigated on a number of other systems. Some systems such as $\mathrm{H}$ or $\mathrm{D} / \mathrm{Ni}(100)^{32}, \mathrm{H} / \mathrm{Ru}(001)^{33}, \mathrm{H}$ or D/Rh(111) ${ }^{34,35}, \mathrm{CO} / \mathrm{Rh}(111)^{35}$, and CO/Pt $(111)^{36}$ do not show much coverage dependence, other systems such as $\mathrm{H}$ or $\mathrm{D} / \mathrm{Ni}(111)^{32}, \mathrm{D} / \mathrm{Pt}(111)^{35}$ and $\mathrm{CO} / \mathrm{Ru}(100)^{37}$ show strong coverage dependence. Most of these studies except CO/Ru(100) did not address the nature of the adsorbate-adsorbate interaction. For $\mathrm{CO} / \mathrm{Ru}(100)$ system the nature of adsorbate-adsorbate interaction was identified as CO-CO direct interaction. However, the use of a lattice gas model for this system with nonequivalent sites diminishes the value of theoretical attempts ${ }^{3,37}$. Without identifying the range of the nearest neighbor interaction, two separate 
calculations, one including CO-CO interaction at saddle points ${ }^{3}$ and the other not $^{37}$, yielded very different inveraction parameters.

In this section n': will try to use a simple model to fit the coverage dependent diffusion results. The simplest theory is the lattice gas model with a mean field approximation as discussed in Chapter II. For CO/Ni(110), the assumptions of the lattice gas model are not strictly met since the adsorption site in $\mathrm{CO} / \mathrm{Ni}(110)$ system changes from a mixture of top and short-bridge sites below a $0.7 \mathrm{ML}$ coverage to pure short-bridge sites above $0.7 \mathrm{ML}$ coverage. The CO-CO direct interaction comes in as a result of the change on adsorption sites. To account for this effect, we modity Eq. (22) of Chapter II by replacing the adsorbate-adsorbate interaction $4 \varepsilon \theta$ and $6 \varepsilon * \theta$ with $2 \varepsilon \theta_{\text {eff }}$ and $2 \varepsilon * \theta$ eff respectively, where $\theta_{\text {eff }}$ is defined by

$$
\begin{aligned}
\theta_{\text {eff }} & =0, & \text { for } \theta<\theta_{0} \\
& =\frac{\theta-\theta_{0}}{1-\theta_{0}} . & \text { for } \theta>\theta_{0}
\end{aligned}
$$

The 4->2, and 6->2 change in the interaction is due to change in the number of nearest-neighbor sites. In our case, the number of nearest-neighbor site of CO$\mathrm{CO}$ direct interaction for $\mathrm{CO} / \mathrm{Ni}(110)$ is 2 when the $\mathrm{CO}$ is at an adsorption site and 4 when $\mathrm{CO}$ is at the saddle point. This modification correctly gives us the CO-CO interaction below $\theta_{0}$ and at $\theta=1$. The $\theta_{0}$ is the coverage at which the adsorption site starts to change. We will take it as the hypothetical value $\theta_{0}=0.67$ for our calculation. The coverage dependent diffusion coefficient is then given by ${ }^{38}$

$$
D(\theta)=D_{0} \exp \left(-E_{a} / k_{B} T\right)(1-\theta) \exp \left(2 \varepsilon \theta_{e f f} / k_{B} T\right) \exp \left(-2 \varepsilon * \theta_{\theta f f} / k_{B} T\right)
$$




$$
x\left\{\frac{1}{1-\theta}+2 \varepsilon \theta_{\text {eff }} / k_{B} T\right\}
$$

The diffusion activation energy and the preexponential factor can be derived from this expression as

$$
\begin{aligned}
& E_{\text {diff }}(\theta)=E_{a}-2 \varepsilon \theta_{\text {eff }}+2 \varepsilon * \theta_{\text {eff }}-\frac{2 \varepsilon \theta_{\text {eff }}(1-\theta)}{1+2 \varepsilon \theta_{\text {eff }}(1-\theta) / k_{B} T} \\
& D_{0}(\theta)=D_{0}\left\{1+2 \varepsilon \theta_{\text {eff }}(1-\theta) / k_{B} T\right\} \exp \left(-\frac{2 \varepsilon \theta_{e f f}(1-\theta) / k_{B} T}{1+2 \varepsilon \theta_{\text {eff }}(1-\theta) / k_{B} T}\right)
\end{aligned}
$$

Assuming $\varepsilon^{*}=0$, we fit the diffusion activation energies by Eq. (16). The solid curves in Fig. $4(\mathrm{a})$ are fittings with parameter $2 \varepsilon=1.8 \mathrm{kcal} / \mathrm{mol}$ in both [110] and [00:] directions. The agreement between the experimental data and the calculation is very reasonable and the resulting interaction energy from the fittings are comnaraule with the reduction of the activation energies, namely $2 \mathrm{kcal} / \mathrm{mol}$. However, the same parameter will not fit $D_{0}(\theta)$. This is because the theory has not considered the effect of the adsorbates on the preexponential factor correctly. Without properly evaluating the entropy change from adsorption sites to saddle points caused by the adsorbate-adsorbate interactions, the model cannot predict a correct preexponential factor. For example, the preexponential factor from Eq. (17) at $\theta=1.0$ is $D_{0}$, which is unreasonable as it is the same as that of tracer diffusion. The compensation effect between the activation energy and the preexponential factor is missing from Eq. (16) and (17), in contradictory with our experimental observation (see Fig. 4). Because of 
this, any attempt to fit $D(\theta)$ as a function of coverage at a constant temperature by Eq. (15) will not result in a correct adsorbate-adsorbate interaction energy. An attempt to fit $D(\theta)$ by Eq. (15), shown by Fig. 5 , leads to an interaction energy $\varepsilon$ about a factor of 3 smaller than the correct one.

\section{Summary}

In summary, we have studied a coverage dependent diffusion for a system with intrinsic anisotropy. Only $\mathrm{CO}-\mathrm{CO}$ direct interaction is found to affect surface diffusion. The adsorbate-substrate interaction and its anisotropy are clearly identified. The CO-CO direct interaction is deduced to be about $2 \mathrm{kcal} / \mathrm{mol}$ and its effect on the anisotropy of the diffusion activation energy appears negligible. The latter has been explained as a result of different adsorbate-adsorbate interactions on the two saddle points. Comparisons with other experimental results have been made and in most cases good agreements have been reached. Theoretical fitting of the coverage dependent diffusion results has been attempted. The crudeness of the model has prohibited us to obtain a complete agreement with the experiment. 


\section{References}

${ }^{1}$ For a recent review, see R. Gomer, Rep. Prog. Phys. 53, 917(1990).

${ }^{2}$ M. Bowker and D.A King, Surf. Sci. 71, 583(1978); 72, 208(1978); D.A.Reed and G. Ehrlich, Surf. Sci. 102, 588(1981); 105, 603(1981); A. Natori and H. Ohstubo, Surf. Sci. 171, 13(1986); R. DiFoggio and R. Gomer, Phys. Rev. B 25, 3490(1982).

${ }^{3}$ V.P. Zhdanov, Surf. Sci. 257, 63(1991), and references therein.

${ }^{4}$ Xu-dong Xiao, Yuanlin Xie and Y. R. Shen, Surf. Sci. 271, 295(1992).

5 M. D. Aley, M. J. Yates, Jr., Surface Sci. 165, 447(1986); D.A.Wesner, F.P.Coenen and H.P.Bonzel, Phys. Rev. Lett. 60, 1045(1988).

${ }^{6}$ W. Riedl and D. Menzel, Surface Sci. 163, 39(1985).

${ }^{7}$ For details, see Xu-dong Xiao, X.D. Zhu, W. Daum and Y.R. Shen, Phys. Rev. B , (1992).

${ }^{8}$ R. Gomer, private communication.

9 D. J. Hannaman and M.A.Passler, Surf. Sci. 203, 449(1988).

${ }^{10}$ B. Voigtlander, D. Bruchmann, S. Lehwald and H. Ibach, Surf. Sci. 225, 151(1990).

${ }^{11}$ S.Haq, J.G.Love and D.A.King, Surf. Sci. 275, 170(1992).

12 J. Bauhofer, M. Hock, and J. Kuppers, Surf. Sci. 191,395(1987).

${ }^{13}$ R. J. Behm, G. Ertl and V. Penka, Surf. Sci. 160, 387(1985).

${ }^{14}$ C.S.Feigerle, S.R.Desai and S.H.Overbury, J.Chem.Phys. 93,787(1990).

${ }^{15}$ H. Kuhlenbeck, M.Neumann and H.-J. Freund, Surf. Sci. 173, 194(1986).

${ }^{16}$ J. Lee, J.Arias, C. Hanrahan, R.Martin, H. Metiu, C. Klauber, M.D.Alvey and J.T.Yates, Jr., Surf. Sci. 159, L460(1985); J.T.Yates,Jr., C. Klauber, M.D. Alvey, H. Metiu, J. Lee, R.M. Martin, J. Arias and C. Hanraman, in: Desorption 
Induced by Electronic Transitions, Proc. DIET II Conf., Eds. W.Brenig and D. Menzel (Springer, Berlin, 1985)p.123; B. A. Gurney and W. Ho, J. Vacuum Sci. Technol. A3, 1541(1985).

${ }^{17}$ Yu.K. Tovbin, Prog. Surf. Sci. 34,1(1991).

18 T.S. Lin, H.-J. Lu and R. Gomer, Surf. Sci. 234, 251(1990).

${ }^{19}$ M. Trenary, K.J.Uram and J. T. Yates,Jr., Surf. Sci. 157, 512(1985).

${ }^{20} \mathrm{H}$. Froitzheim and U. Kohler, Surf. Sci. 188,70(1987); J.B.Miller, H.R.Siddiqui, S.M.Gates, J.N.Russell,Jr., J.T. Yates,Jr., J.C.Tully, and M.J.Cardillo, J.Chem.Phys. 87, 6725(1987).

${ }^{21}$ B. Roop, A. Costello, D. R. Mullins, and J. M. White, J. Chem. Phys. 86, 3003(1987).

22 H. C. Peebles, D. E. Peebles and J. M. White, Surface Sci. Lett. 125, L87(1983).

23 J.T.Yates,Jr., and D. W.Goodman, J. Chem.Physics, 73, 5371(1980).

${ }^{24}$ T. Narusawa, W. M. Gibson, and E. Tornqvist, Phys. Rev. Lett. 47, 417(1981); Surf. Sci. 114, 331(11982).

${ }^{25}$ W. Oed, U. Starke, K. Heinz, K. Muller, and J. B. Pendry, Surf. Sci. 251/252, 488(1991).

${ }^{26}$ M. L. Xu, and S. Y. Tong, Phys. Rev. B 31, 6332((1985); S. M. Yalisove, W. R. Graham, E. D. Adams, M. Copel, and T. Gustafsson, Surf. Sci. 171, 400(1986).

${ }^{27}$ M. W. Finnis, and V. Heine, J. Phys. F 4, L37(1974).

${ }^{28}$ G. Doyen and G. Ertl, Surf. Sci. 43, 197(1974).

${ }^{29}$ For example, C. Kittle, Introduction to Solid State Physics, (John Wiley \& Son, Inc. New York, 1986).

${ }^{30}$ Peter Hanggi, Peter Talkneer, and Michal Borkovec, Rev. Modern Phys. 62, 251(1990). 
${ }^{31}$ C. H. Mak and S. M. George, Chem. Phys. Lett. 135, 381(1987).

32 T.-S. Lin and R. Gomer, Surf. Sci. 255, 41(1991).

${ }^{33}$ C.H.Mak, J. L. Brand, B.G.Koehler, and S.M.George, Surface Sci. 191,108(1987).

${ }^{34}$ S.S.Mann, T.Seto, C.J.Barnes and D.A.King, Surface Sci. 261, 155(1992).

${ }^{35}$ E. G. Seebauer, A.C.F. Kong and L.D.Schmidt, J. Chem. Phys. 88, 6597(1988).

${ }^{36}$ V.J.Kwasniewski and L.D. Schmidt, Surf. Sci. 274, 329(1992).

${ }^{37}$ A.A.Deckert, J. L. Brand, M. V. Arena and S. M. George, Surface Sci. 208,441(1989); V.P.Zhdanov, Surface Sci. 257, 63(1991).

${ }^{38}$ V. P. Zhdanov, Phys. Lett. A 137, 409(1989). 
Figure captions:

Figure 1: Linear diffraction signals versus time for coverage $\theta=0.98$ at three temperatures for diffusion (a) along [110], and (b) along [001]. The solid lines are single exponential fits.

Figure 2: Diffraction signals versus time with a second laser pulse applied at the times indicated by the arrows. The experiment have been carried out along [001] at $T=210 \mathrm{~K}$ for initial coverage $\theta_{0}=0.5$. The starting $S / N$ is $\sim 40$, and the second laser pulse intensities are: (a)1.08J/ $\mathrm{cm}^{2}$, (b) $0.98 \mathrm{~J} / \mathrm{cm}^{2}$, and (c) $1.11 \mathrm{~J} / \mathrm{cm}^{2}$. The time constants indicated along the decay signals are from single exponential fits.

Figure 3: Arrhenius plot for diffusion of $\mathrm{CO}$ on $\mathrm{Ni}(110)$ with coverage $0.48 \mathrm{ML}$ and $0.98 \mathrm{ML}$ along the two principal directions: [1 $1 \overline{1} 0]$ and [001].

Figure 4: (a) Diffusion activation energy and (b) preexponential factor as a function of coverage for $\mathrm{CO}$ diffusing along [110] and [001] directions. The solid lines in (a) are theoretical fits by Eq. (16), and the solid lines in (b) are for eyeguide. The desorption energy as a function of coverage has also been depicted in (a) for comparison.

Figure 5: Diffusion coefficient as a function of coverage at constant temperatures. The solid lines are theoretical fits by Eq.(15) with $2 \varepsilon=0.55 \mathrm{kcal} / \mathrm{mol}$ for $218 \mathrm{~K}, 0.61 \mathrm{kcal} / \mathrm{mol}$ for $200 \mathrm{~K}$, and $0.63 \mathrm{kcal} / \mathrm{mol}$ for $182 \mathrm{~K}$ respectively. 
Figure 6: Geometries of $\mathrm{CO}$ on Ni(110) surface with one Ni site unoccupied. The positions of diffusion saddle points along [1 $1 \overline{1} 0]$ and along [001] has been indicated in (a) and (b) with the relevant distances labeled. The $\mathrm{Ni}$ and $\mathrm{CO}$ are not drawn in proportion.

Figure 7: Surface potential corrugations given by Eq. (8). The scales of the barrier height in [1 10$]$ and [001] are not in proportion for the purpose of showing anisotropy. 


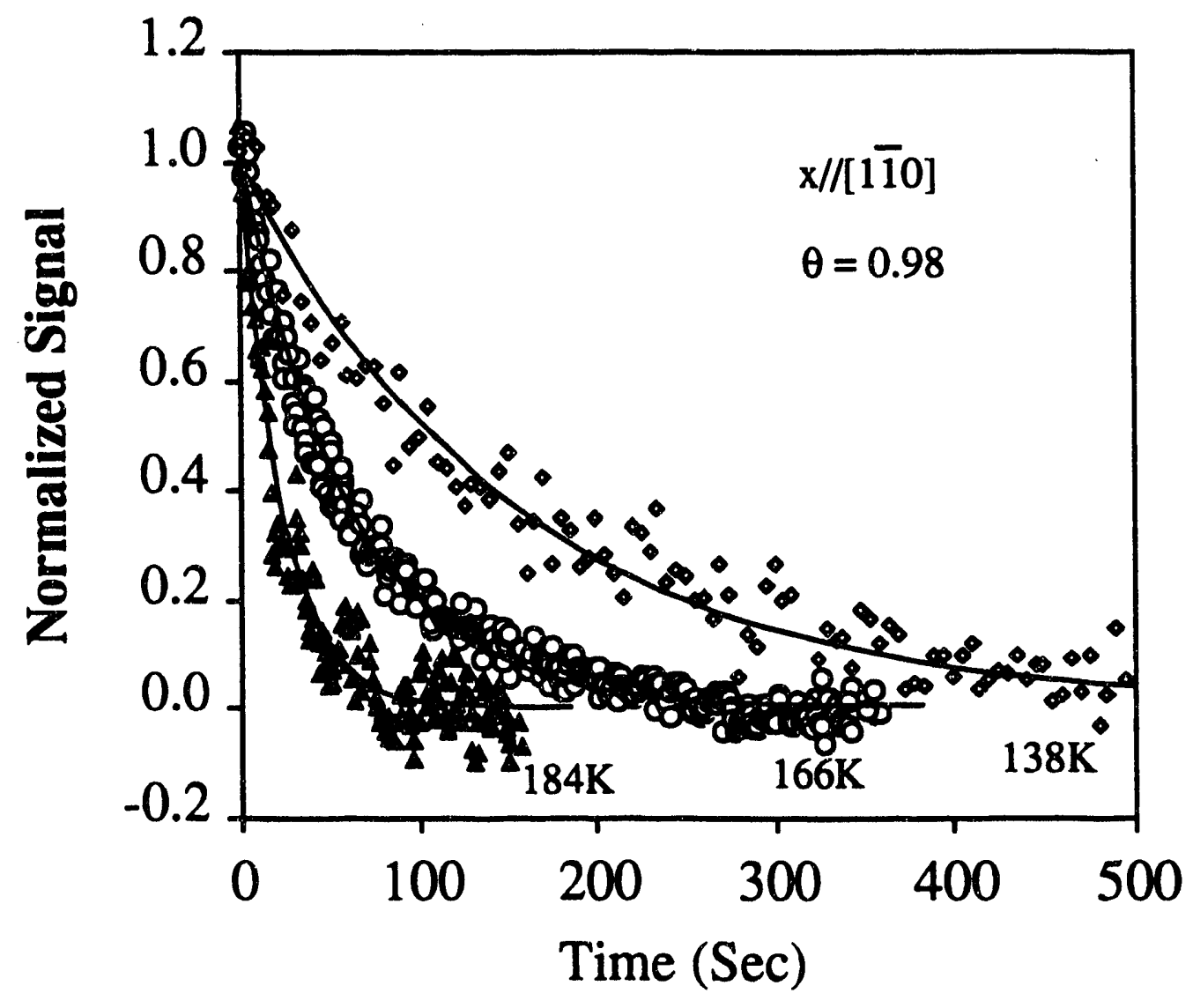

Figure 1(a) 


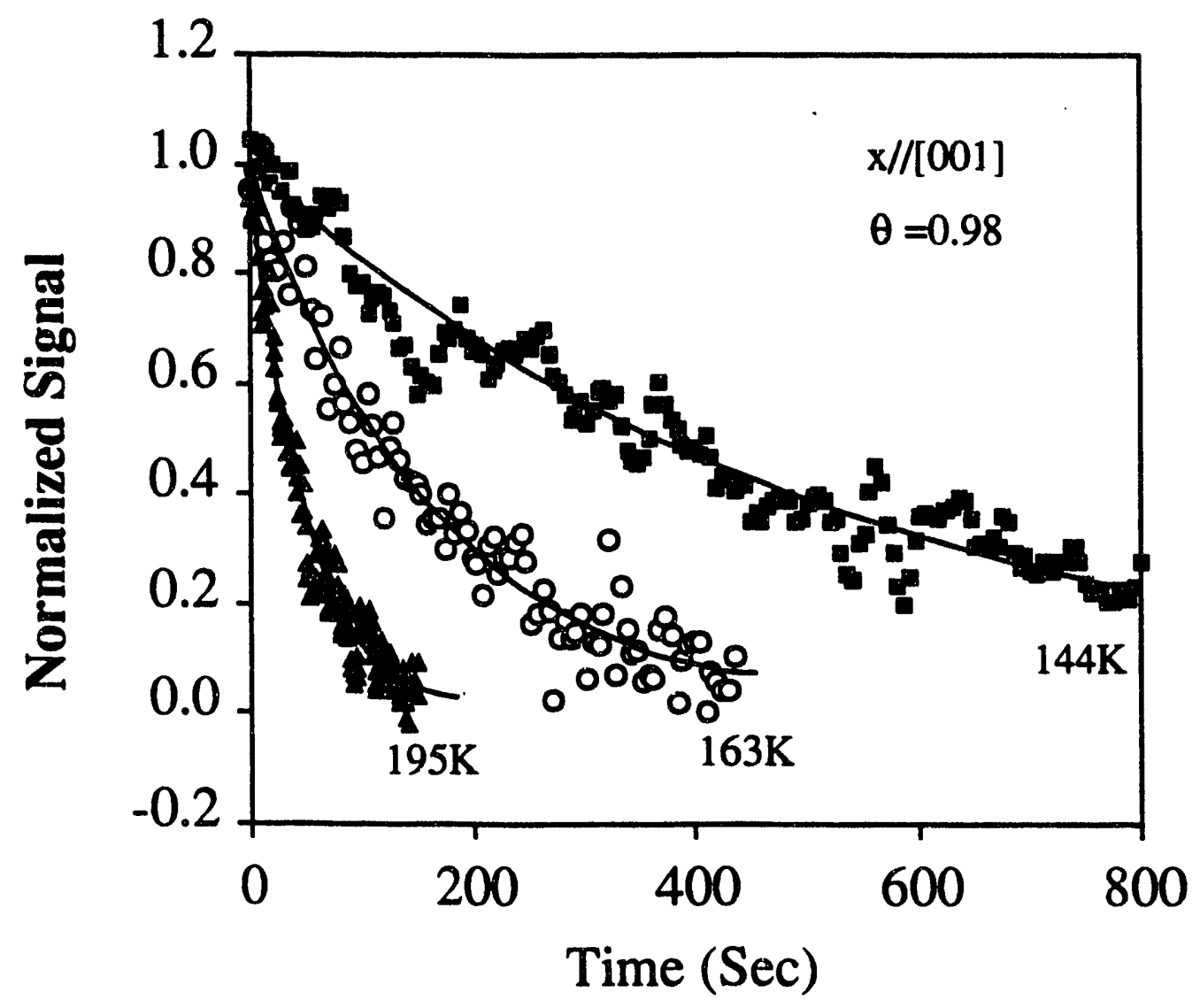

Figure 1(b) 


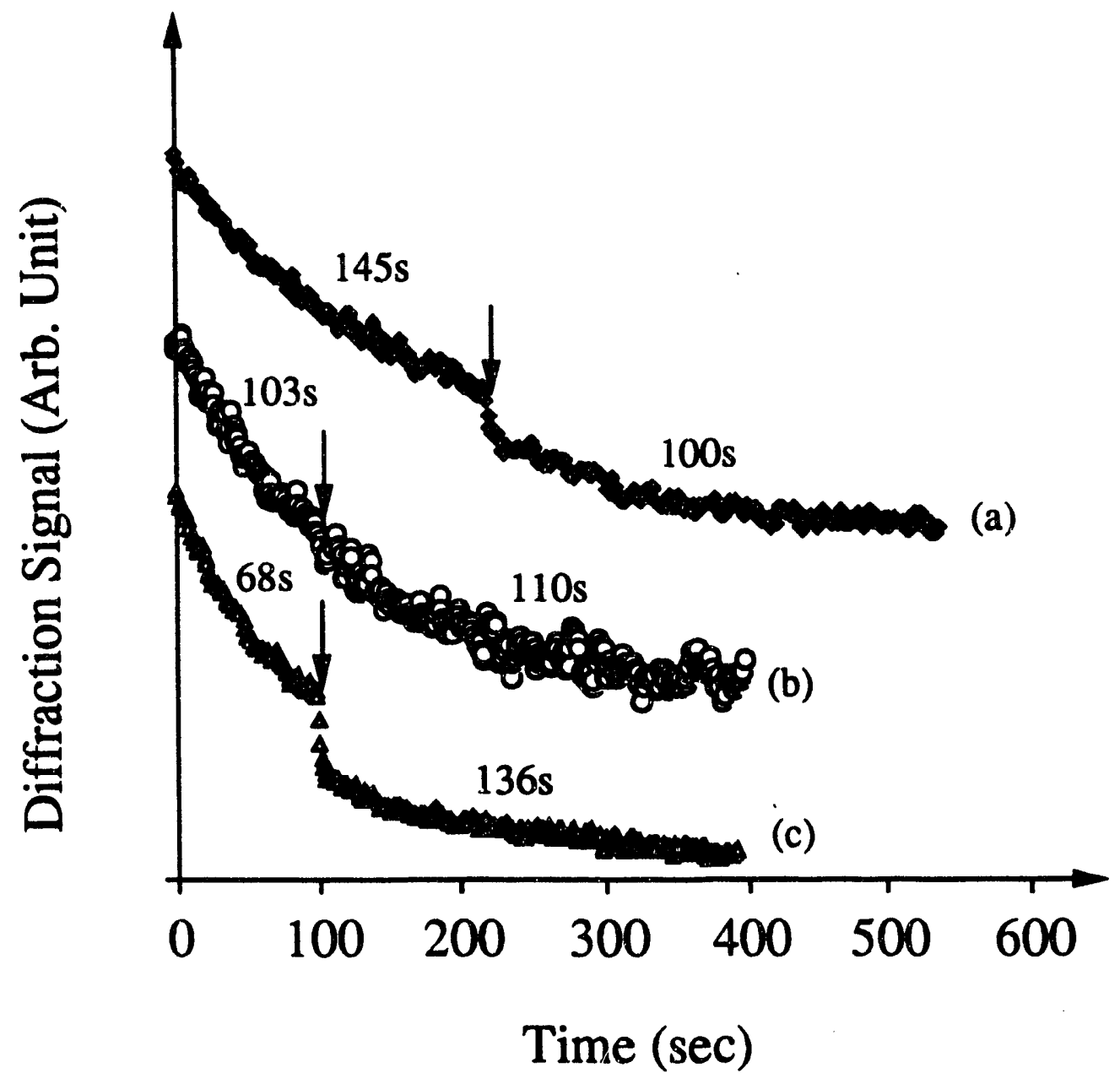

Figure 2 


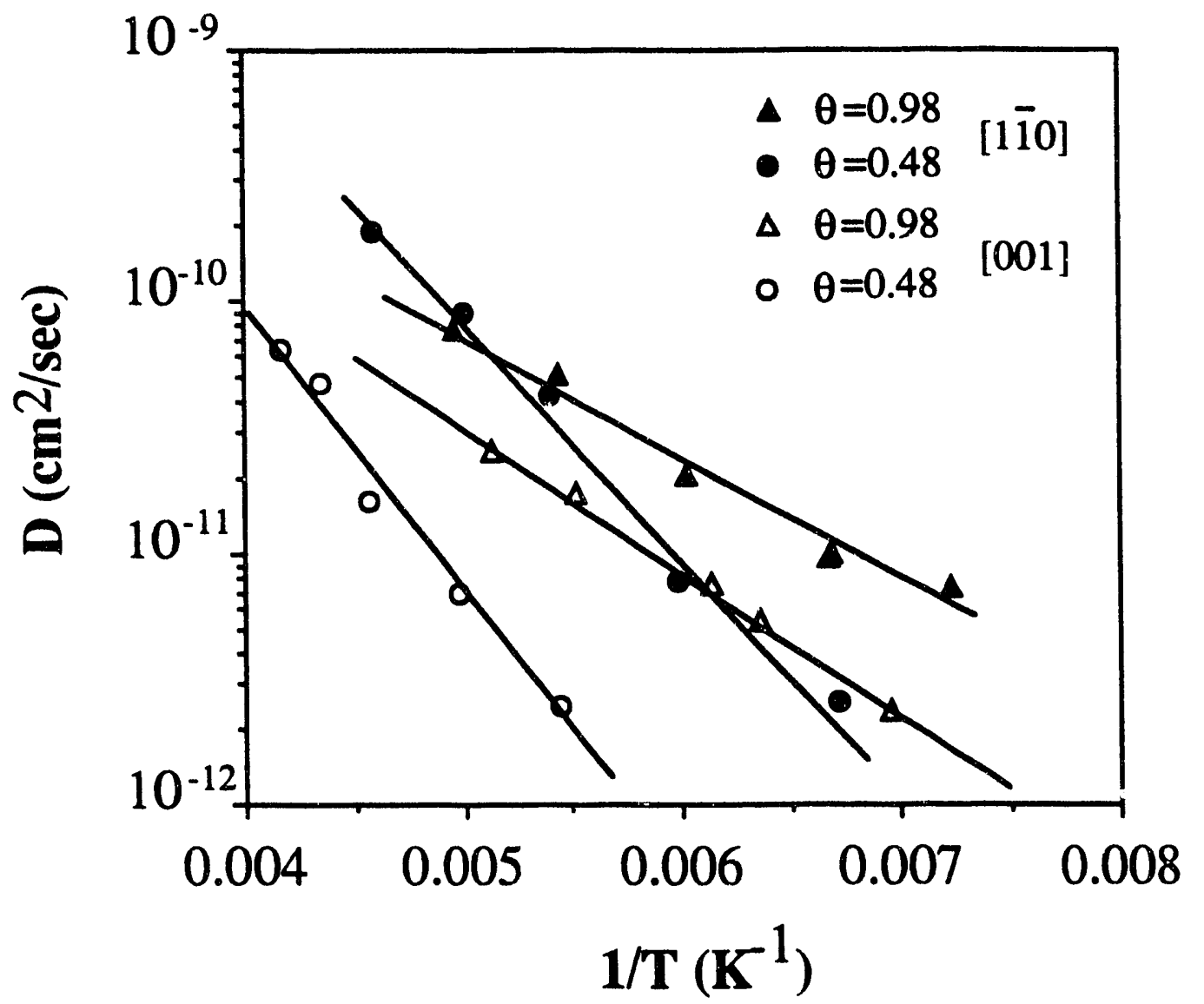

Figure 3 


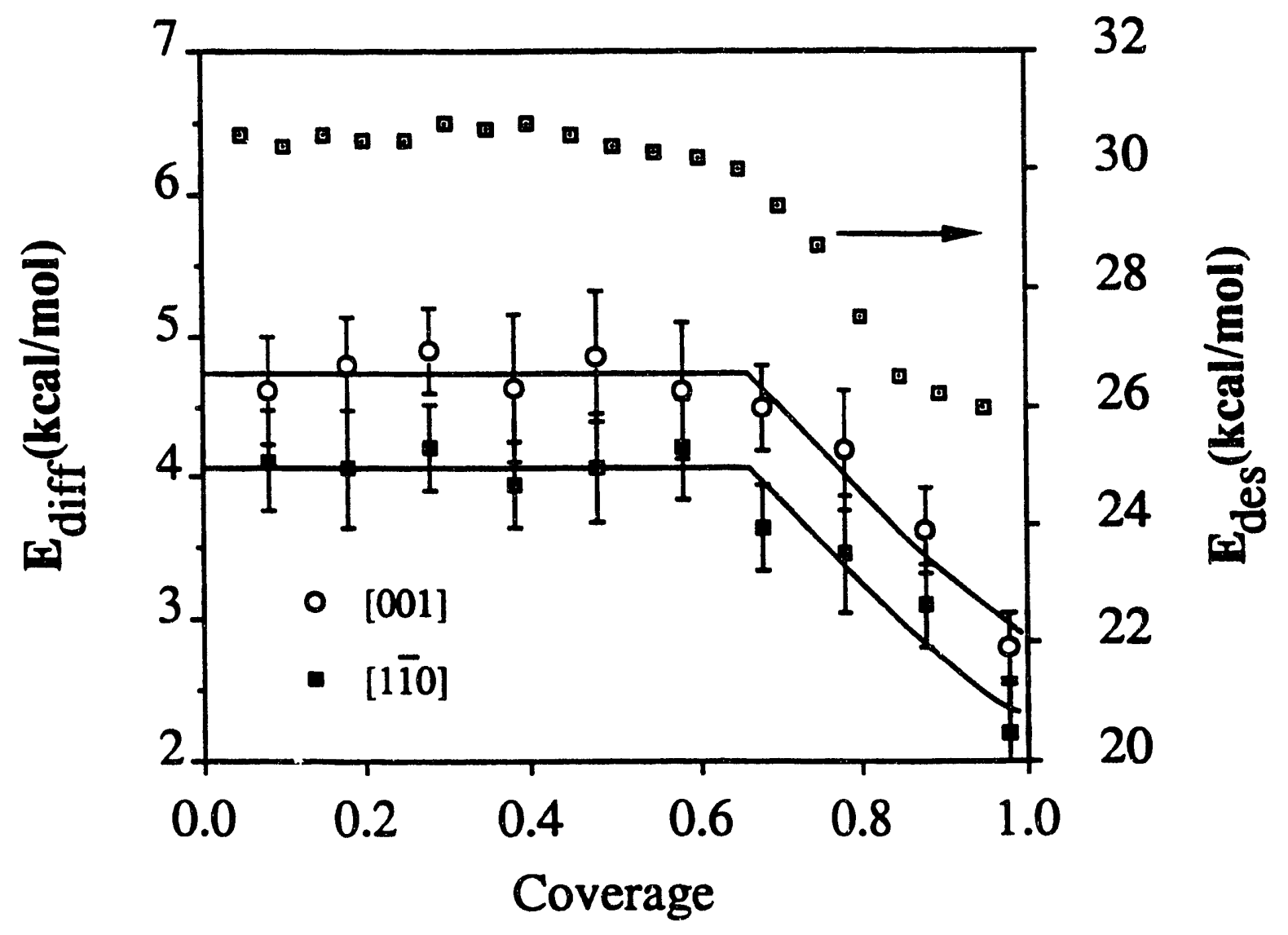

Figure 4(a) 


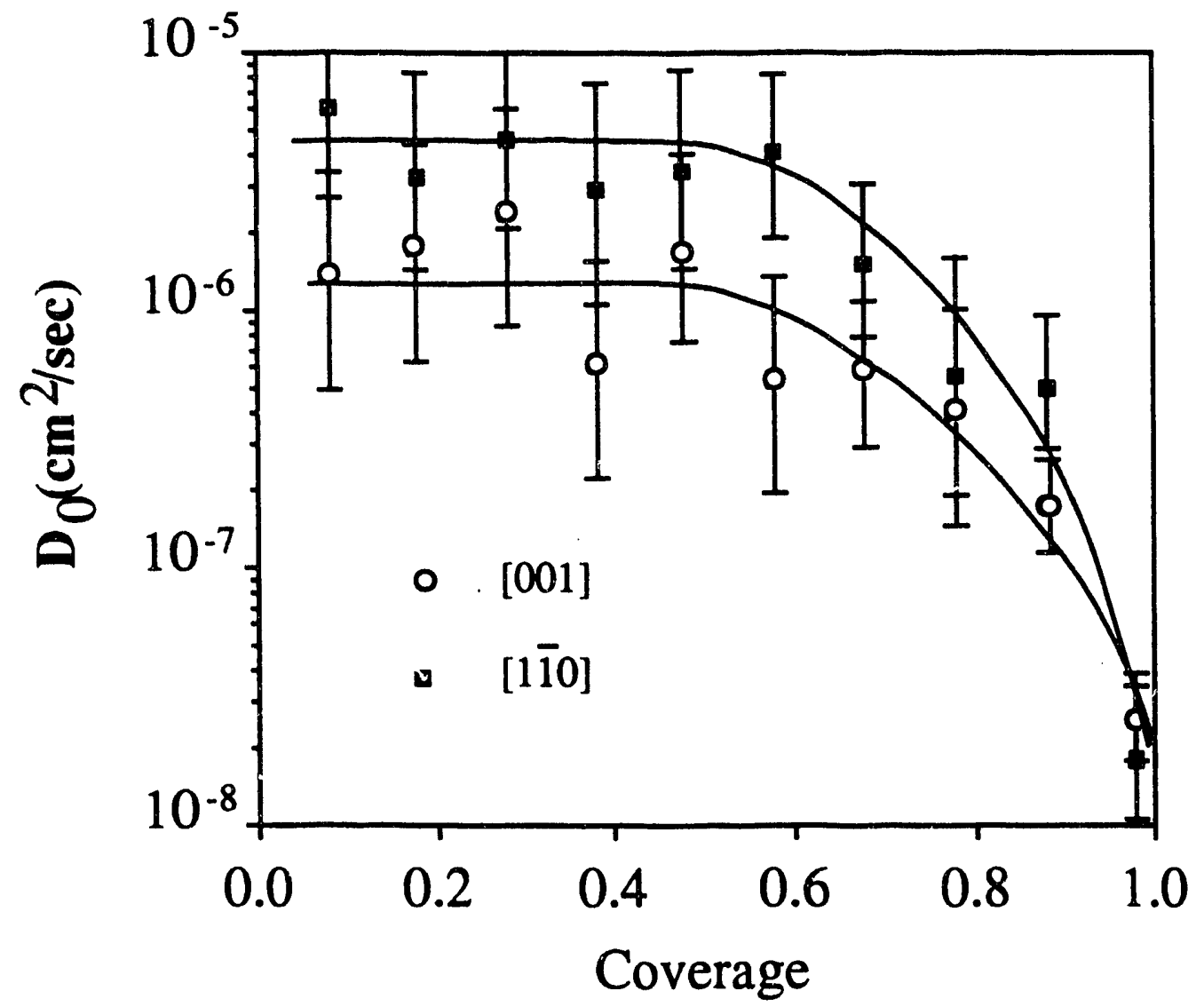

Figure 4(b) 


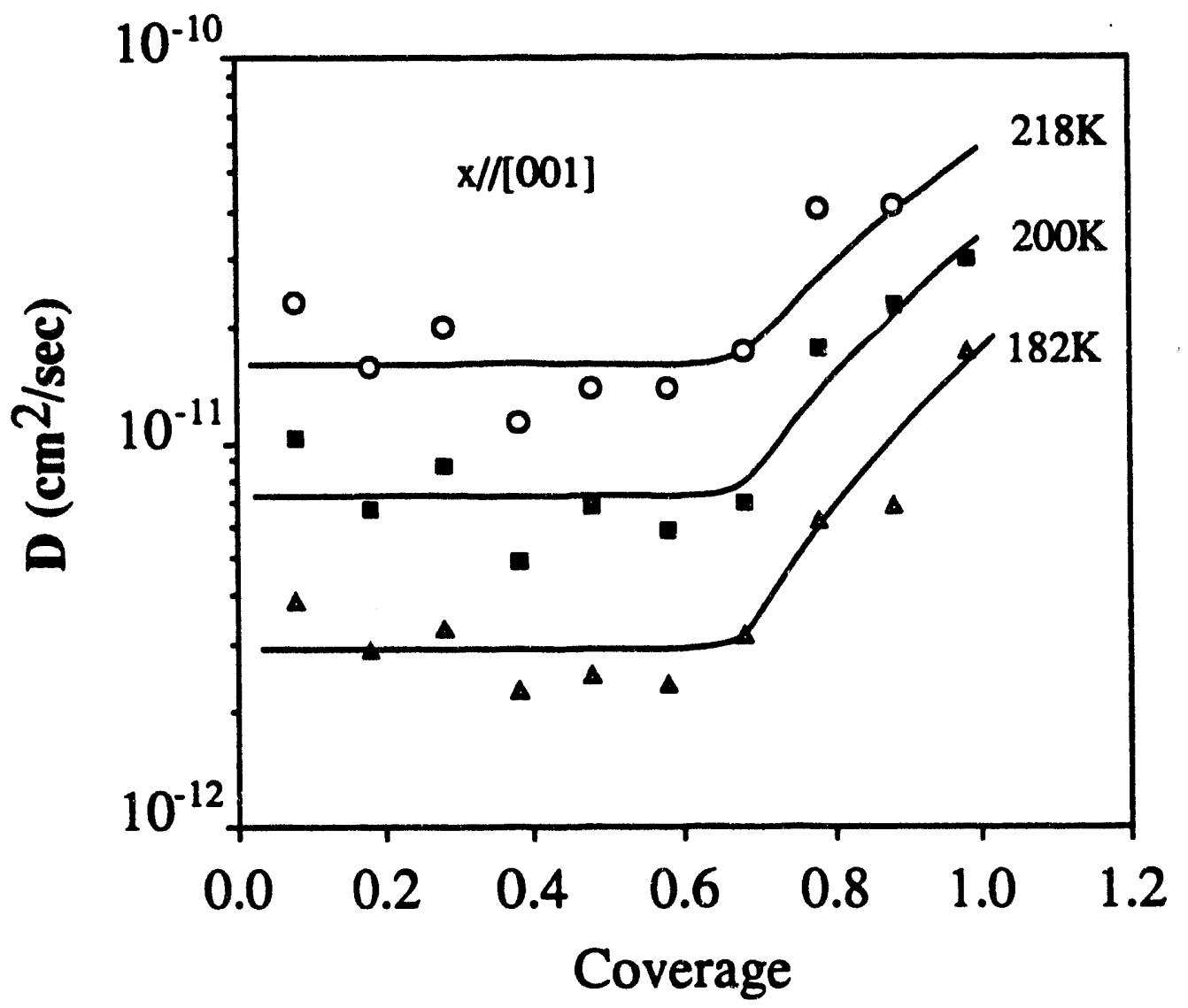

Figure 5 


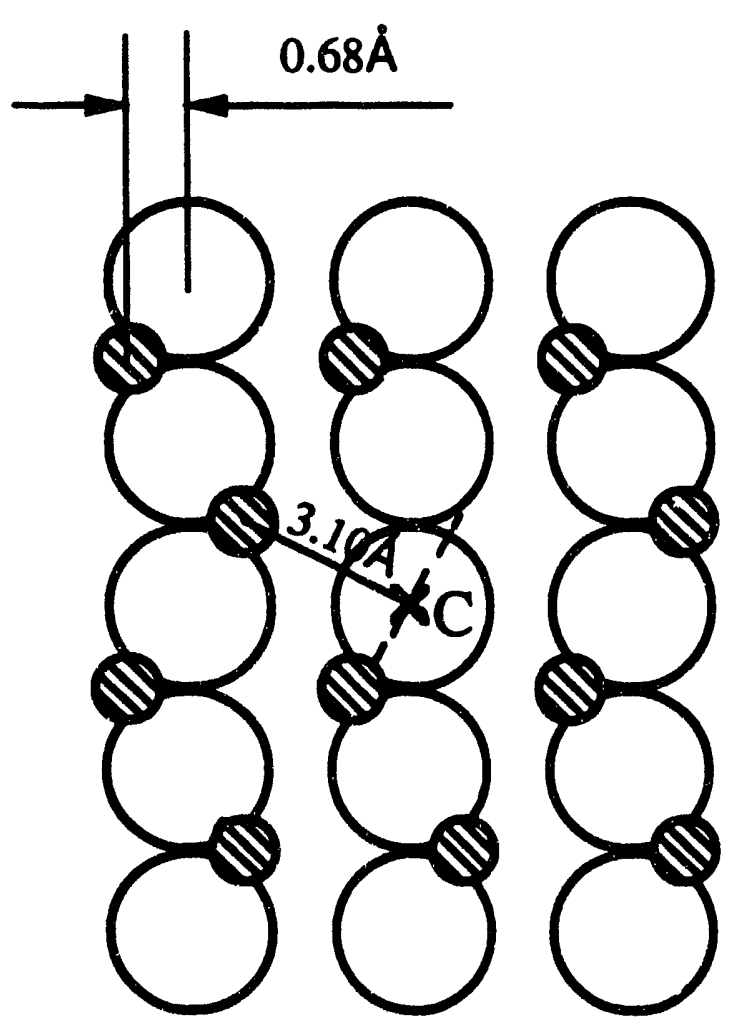

(a)

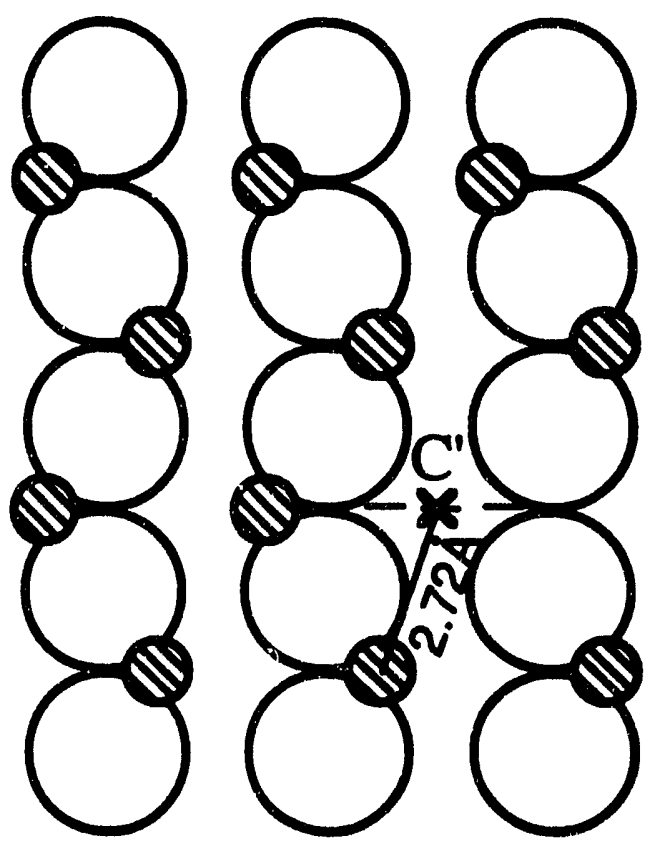

(b)

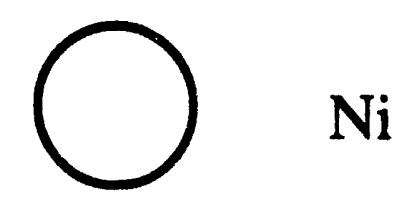

Figure 6 


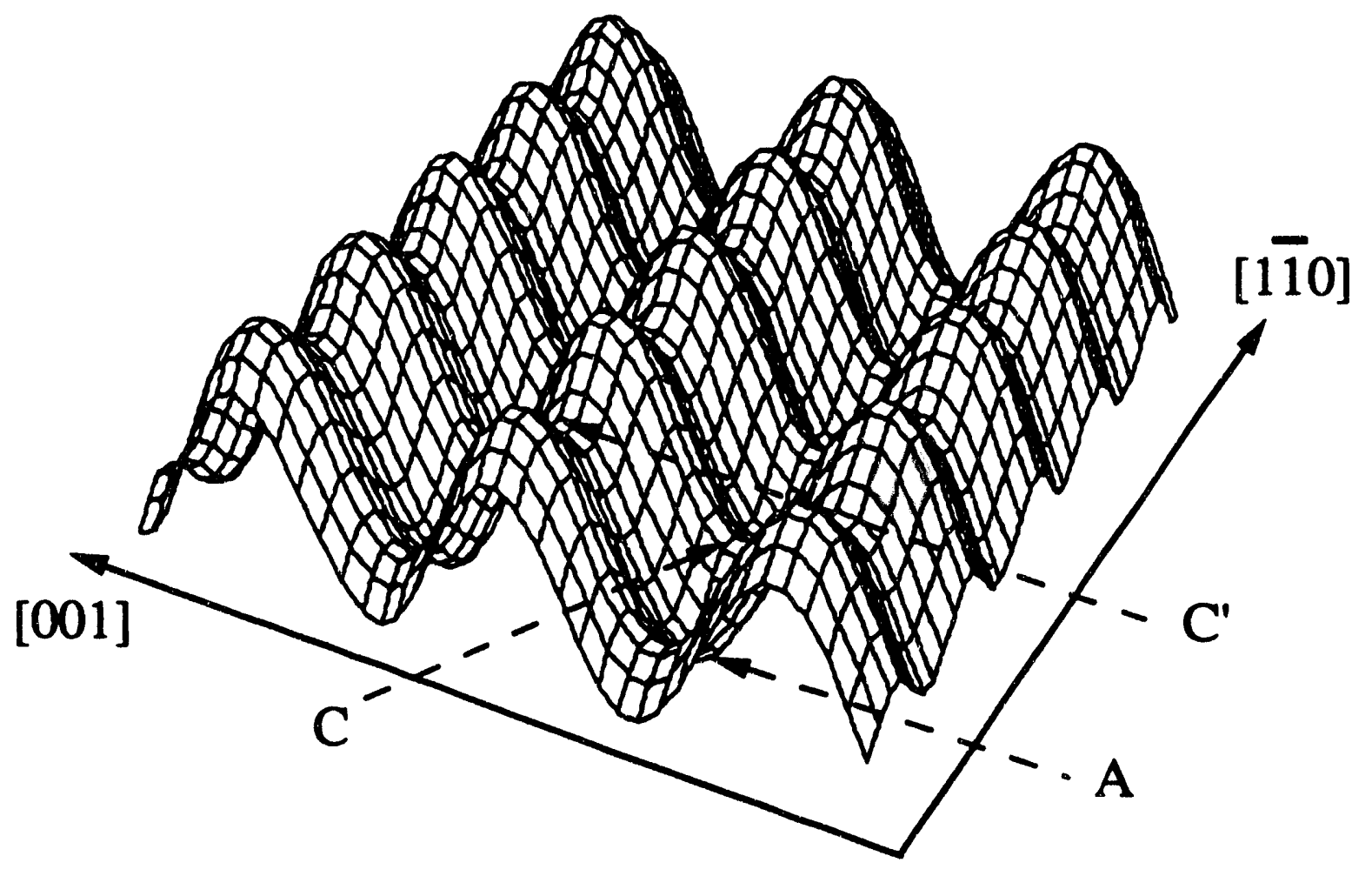

Figure 7 


\section{Impurities, Defects, and Surface Diffusion: CO/NI(110)}

\section{A. Introduction}

As an important surface process surface diffusion has been studied extensively with a variety of techniques in the past ${ }^{1}$. Considerable data base was established for different systems. However, questions such as how impurities and defects affect surface diffusion were seldom explored experimentally. Theoretical calculations on these questions do exist. They predict little effect from small amount of impurities or defects (few percent) ${ }^{2,3}$.

It has been noted that surface diffusion measurements carried out presumably on the same systems with different techniques by different authors often yield rather different activation energies and preexponential factors ${ }^{1,4}$ The discrepancy was often attributed to difference in temperatures ${ }^{1}$ or techniques ${ }^{5}$ used in the measurements. Whether this discrepancy can also be due to different samples and different sample treatments, or more specifically, due to surface impurities, defects, or strains, no one has yet provided any answer.

Recently we have worked on $\mathrm{CO}$ diffusion on $\mathrm{Ni}(110)$ surface using different samples and different sample preparation procedures. The results showed unambiguously that the sample treatment could significantly affect surface diffusion. In this chapter we will report effects from $S$ impurities and surface defects on $\mathrm{CO}$ diffusion on $\mathrm{Ni}(110)$. The investigation on the effect of $\mathrm{S}$ impurities was performed for a few low $S$ coverages and it was found that as low as $3 \% \mathrm{~S}$ monolayer could change appreciably the diffusion speed and its activation energy of $\mathrm{CO}$ on $\mathrm{Ni}(110)$. The effect of surface point defects was examined in a qualitative way, namely by additional $\mathrm{Ar}^{+}$ion sputtering on a 
well annealed surface or by annealing an $\mathrm{Ar}^{+}$sputtered sample for different length of times. The step effect on $\mathrm{CO}$ diffusion was carried out with a miscut $\mathrm{Ni}(110)$ surface. The effect of point defects was found to be profound in altering the preexponential factor, but insignificant to the diffusion activation energy. The steps have been found to dominate surface diffusion with an increasing surface diffusion barrier. Qualitative explanations are given for the observations.

\section{B. Experiment}

The experiment was performed in an ultrahigh vacuum (UHV) chamber with a base pressure of $2.0 \times 10^{-10}$ torr. The major set of the data was collected from a single crystal $\mathrm{Ni}(110)$ sample, cut and mechanically polished to within $0.2^{\circ}$ from the (110) plane. The data on the step effect were obtained from a $\mathrm{Ni}(110)$ sample risscut by $\sim 1.5^{\circ}$ along the [110] direction. Before any measurement, the sample was treated by many sputtering and annealing cycles to get rid of impurities such as $S, O$, and $C$. Subsequently, normal cleaning procedure was adopted to prepare a clean surface, i.e., the surface of the sample was first $\mathrm{Ar}^{+}$sputtered at $1.0 \times 10^{-4}$ torr Ar pressure with a $500 \mathrm{~V}$ beam voltage for approximately 30 minutes at room temperature and then annealed at $1120 \mathrm{~K}$ for $10 \mathrm{~min}$ followed by a slow cooling down at a rate of $\sim 0.5 \mathrm{~K} / \mathrm{sec}$ to $800 \mathrm{~K}$ and a rate of $2 \mathrm{~K} / \mathrm{sec}$ to room temperature. In the later sections, surfaces prepared by this procedure will be referred as normally prepared surface. Auger spectra showed no detectable impurities (<0.3\% S and C ). Sharp $1 \times 1$ LEED pattern from a clean $\mathrm{Ni}(110)$ surface and clear $2 \times 1$ LEED pattern from a full CO monolayer on $\mathrm{Ni}(110)$ were observed to ensure that the surface was well ordered. Liquid nitrogen was used to cool the sample afterwards to the measurement temperatures. A Chromel-Alumel thermal couple welded to the 
sample was used to monitor the sample temperature. The temperature could be controlled to within $\pm 1 \mathrm{~K}$.

For experiment on the $S$ impurity effect, the $S$ surface impurities were introduced through bulk-to-surface segregation by heating a normally prepared sample for an extended period at $1120 \mathrm{~K}$, typically, about one hour to yield $1 \%$ $S$. The concentration of $S$ was measured by AES with a calibrated ratio of $\mathrm{S}(152 \mathrm{eV}) / \mathrm{Ni}(848 \mathrm{eV})^{6}$. The $\mathrm{S}$ atoms are known to desorb only at very high temperatures (above 1200K) ${ }^{7}$ and are presumably uniformly distributed on the $\mathrm{Ni}(110)$ surface due to the S-S repulsive interactions. Therefore briefly flashing sample to $600 \mathrm{~K}$ to get rid of either the adsorbed ambient gas molecules or the previously adsorbed $\mathrm{CO}$ before each diffusion measurement should not alter the amount of $S$. The experiment on the effect of point defects was performed in two different ways. The first one was by annealing the sample at $1120 \mathrm{~K}$ for different lengths of time, from less than 1 minute to 10 minutes, after it is sputtered by $\mathrm{Ar}^{+}$for 30 minutes. The second one was by sputtering a normally prepared $\mathrm{Ni}(110)$ surface by a $500 \mathrm{eV} \mathrm{Ar}^{+}$beam with a current density of $5 \mu \mathrm{A} / \mathrm{cm}^{2}$ at room temperature for $5 \mathrm{~min}$ without no further annealing.

With the Ni(110) sample prepared in the above ways, we then dosed CO to a saturation coverage. LEED structures were still found to be $2 \times 1$ for all the surfaces except for the $15 \%$ S surface, where a $2 \times 1$ structure was barely visible with a large diffused background. CO diffusion along [110] was chosen to be investigated and was measured at least at four different temperatures in the range of $140 \mathrm{~K}-220 \mathrm{~K}$ on each prepared surface. The measurement technique was described in Chapter III and we give only a briefly review here. First, two $1.06 \mu \mathrm{m}$ laser beams were used to interfere at the $\mathrm{CO}$ covered $\mathrm{Ni}$ surface to create an adsorbate grating with a coverage modulation of $\sim 3 \%$ and a grating spacing of $\sim 3 \mu \mathrm{m}$. Then a polarization modulated $\mathrm{He}-\mathrm{Ne}$ laser beam was used 
to probe the adsorbate grating with an incident angle of $45^{\circ}$ and the first order diffraction signal was monitored as a function of time by a lock-in detection scheme. The decay of the first order diffraction signal is related to the surface diffusion coefficient $D$ by

$$
S_{1}=S_{01} \exp \left(-8 \pi^{2} D t / s^{2}\right)
$$

where $s$ is the grating spacing and $S_{01}$ is the initial diffraction signal strength. It should be noticed that the decay time constant here depends only on the grating spacing but not no its shape.

\section{Results and Discussion}

\section{a) S Impurity Effect}

In Fig. 1 we show the measured CO diffusion coefficients along [1ํㅣ] as a function of reciprocal temperature in an Arrhenius plot for a number of differently prepared surfaces with a $\mathrm{CO}$ average coverage of $\theta \sim 0.98$. Data (a) is from a normally prepared surface as described in the previous section. Data (b), (c), $(d)$, and $(e)$ are from surfaces with different amount of $S$ impurities. The Arrhenius fitting parameters for these cases are summarized in Table 1. It is seen that the CO diffusion activation energy on $\mathrm{Ni}(110)$ with $\leq 1 \% \mathrm{~S}$ impurity remain unchanged $(2 \mathrm{kcal} / \mathrm{mol})$ within the experimental error. However, the $\mathrm{CO}$ diffusion activation energies on surfaces with $3 \%$ or higher S coverage are clearly much higher: about $6 \mathrm{kcal} / \mathrm{mol}$ for surfaces with $3 \%$ and $5 \% \mathrm{~S}$ coverages and $8 \mathrm{kcal} / \mathrm{mol}$ for surface with $15 \% \mathrm{~S}$. Although the preexponential factors are 
significantly larger with impurities than that for a clean surface, the $\mathrm{CO}$ diffusion speeds are much slower than that on a clean surface in the temperature range of our measurement. Especially in the low temperature region ( 160K) the CO diffusion on surfaces with $5 \%$ S impurity are 2 3 orders of magnitude slower.

The above results are surprising in many folds. First, with only a few percent of S impurity on the Ni surface the $\mathrm{CO}$ diffusion has been dramatically impeded in the low temperature regime. The barrier for $\mathrm{CO}$ diffusion has also been significantly altered. Second, the change of $\mathrm{CO}$ diffusion activation energy seems to have a sudden jump as the $\mathrm{S}$ impurity on Ni(110) surface increases. A careful investigation of this may provide information about the nature of the $S$ contaminated $\mathrm{Ni}(110)$ surface.

If sulfur is only considered as a usual coadsorbate of $\mathrm{CO}$, the existing theory cannot predict such a dramatic change in the $\mathrm{CO}$ diffusion coefficient with any realistic S-CO interactions ${ }^{2}$. What is responsible for the change, in our opinion, must be associated with the $\mathrm{Ni}$ substrate. It is known that $\mathrm{S}$ acts as a poison on $\mathrm{Ni}$ for catalytic methanation reaction ${ }^{9,10}$. The surface science studies of $\mathrm{S} / \mathrm{Ni}(110)$ has revealed that $\mathrm{S}$ atoms adsorb at the rectangular hollow sites of the $\mathrm{Ni}(110)$ surface and form a $p(2 \times 2)$ superstructure at $0.25 \mathrm{ML}$ coverage, and a $c(2 \times 2)$ superstructure at $0.5 \mathrm{ML}^{11}$. Sulfur has a strong interaction with $\mathrm{Ni}$ surfaces and this interaction generally leads to an expansion of the Ni surface. With a saturation $\mathrm{S}$ coverage $(0.5 \mathrm{ML}, \mathrm{c}(2 \times 2))$, the originally contracted $\mathrm{Ni}(110)$ surface $\left(\Delta d_{12} / d_{12 B} \sim-9 \%, d_{12 B}\right.$ is the bulk distance between two layers in the surface normal direction) is expanded by more than $10 \%$ as compared to the bulk atomic distance ${ }^{11}$. For a clean metal surface, contractive relaxation presumably results from smoothing of surface electronic charge density ${ }^{12}$. Expansion of $20 \%$ of the first $\mathrm{Ni}$ layer at an $\mathrm{S}$ saturation coverage could significantly increase the electronic charge density corrugation and thus 
the surface potential corrugation. Although no measurement exists on how this first $\mathrm{Ni}$ layer of $\mathrm{Ni}(110)$ expands as $\mathrm{S}$ coverage increases, experiment on $\mathrm{S} / \mathrm{Ni}(100)$ system seems to indicate that the substrate expands linearly as a function of the $\mathrm{S}$ coverage ${ }^{13}$. In the case of $\mathrm{Ni}(110)$, we suspect that considerable first Ni layer expansion can occur at low $S$ coverages so that a large number of $\mathrm{CO}$ adsorption sites are affected. Despite some controversy ${ }^{14}$, it was suggested that for a $\mathrm{S} / \mathrm{Ni}(100)$ surface each $\mathrm{S}$ atom may have effectively poisoned $10 \mathrm{Ni}$ atoms ${ }^{10,15}$. Theoretical calculation on $\mathrm{S} / \mathrm{Rh}(100)$ also indicated that the effect of $\mathrm{S}-\mathrm{Ni}$ interaction can be extended to the next nearest neighbors of the $S$ adsorption site (hollow site) through the local density of electronic state at the Fermi energy ${ }^{16}$. For $\mathrm{S} / \mathrm{Ni}(110)$, if we assume that the local Ni expansion induced by $S$ is independent of coverage, then this distortion has to be relaxed by the nearby $\mathrm{Ni}$ atoms. Consequently, it is possible that one adsorbed $\mathrm{S}$ atom can affect its nearest four and the next nearest neighbor eight $\mathrm{Ni}$ atoms. With this picture in mind, a $3 \%$ S coverage could have affected more than $30 \%$ of the $\mathrm{Ni}(110)$ surface. With $\mathrm{CO}$ diffusing on such a modified, more corrugated $\mathrm{Ni}(110)$ surface, the CO diffusion energy barrier can be effectively higher, as indicated by our measurement.

The sudden jump in the diffusion activation energy with coverage of $S$ could be due to the fact that a critical coverage of $S$ can effectively induce a surface corrugation change over the entire surface. Whether this is true or not can be checked by a dynamic LEED analysis as a function of $S$ coverage.

\section{b) Defects Effect}

The data $(f)$ and $(g)$ in Fig. 1 were taken on surfaces with different defect densities for $C O$ coverage $\theta=0.98$. In the case of $(f)$, a Ni(110) surface was 
flash-annealed at $1120 \mathrm{~K}$ for $<1 \mathrm{~min}$ followed by a cooling down procedure described in section $\mathrm{B}$. In the case of (g), a normally prepared Ni(110) surface, which was annealed at $1120 \mathrm{~K}$ for $10 \mathrm{~min}$, was $\mathrm{Ar}^{+}$sputtered for $5 \mathrm{~min}$.

Comparing to the normally prepared surface (a), CO diffusions in cases ( $f$ ) and (g) are significantly faster. However, the diffusion activation energies remain the same as that of a normal surface within the experimental error (Table 1).

In order to explain the $\mathrm{CO}$ diffusion data with defects on the $\mathrm{Ni}(110)$ surface, we need to know the defects density on the surfaces. Although experiment showed that a saturation density of defects created by $\mathrm{Ar}^{+}$ sputtering exists ${ }^{17}$, no absolute knowledge of this value is known since the techniques that have been employed in measuring this quantity can only provide relative information. In principle, the annealing process should remove the surface defects and lead to a microscopic smooth and flat surface as long as the annealing temperature is lower than the surface roughening temperature and the annealing time is long enough ${ }^{18}$. However, the healing speed and its temperature dependence are seldom known. In the literature, annealing temperatures in the range of $1000-1300 \mathrm{~K}$ for preparing a $\mathrm{Ni}(110)$ surface have been often reported without providing the annealing time ${ }^{19}$, not to speak about the residual defect density. For $\mathrm{Pt}(110)$, which has a surface roughening temperature of $1080 \mathrm{~K}^{20}$, it has been found that as much as $40 \mathrm{~min}$ annealing at $1000 \mathrm{~K}$ is required to remove the defects and create a smooth flat surface (judging by the $X$-ray diffraction pattern to have an average step spacing of $500 \AA)^{21}$. In the case of $\mathrm{Ni}(110)$, the surface roughening has been studied by high resolution LEED and a roughening transition temperature of $1300 \mathrm{~K}$ is found ${ }^{22}$. Annealing $\mathrm{Ni}(110)$ at $1120 \mathrm{~K}$ should eventually lead to a microscopically smooth surface. The annealing time at this temperature can be estimated by the power law of the terrace growth kinetics ${ }^{23}$, 


$$
L=A(T) t^{n}
$$

where $L$ is the dimension of terrace, $A(T)$ is a temperature dependent rate coefficient, and the exponent $n$ is found to be $\sim \frac{1}{2}$ by at high temperatures ${ }^{21}$.

Knowing that $A(T)$ is proportional to the self-diffusion coefficient $D(T)$ and $D(T \sim 1120 \mathrm{~K}) \sim 3 \times 10^{-6} \mathrm{~cm}^{2} / \mathrm{sec}$ for $\mathrm{Ni}(110)^{24}$, and $\mathrm{D}(\mathrm{T} \sim 100 \mathrm{~K}) \sim 5 \times 10^{-7} \mathrm{~cm}^{2} / \mathrm{sec}$ for $\mathrm{Pt}(110)^{25}$, the annealing time for getting a smooth $\mathrm{Ni}(110)$ surface is then in the order of minutes at $1120 \mathrm{~K}$. From the above argument, we conclude that the defect densities increases as we go from (a) to $(f)$ and to $(g)$.

Although in the surface treatment of (a) and (f), two processes could happen simultaneously during surface annealing, one being the healing of defects, the other being the segregation of $S$ atoms to the surface with time, we could safely exclude the possible $S$ impurity effect since it has an extremely low surface density $(<0.3 \%)$. Knowing that there were increasing surface defect densities for surfaces (a), (f), and (g), our experimental results show unambiguously that $\mathrm{CO}$ diffusion along $[1 \overline{1} \mathrm{1}]$ on $\mathrm{Ni}(110)$ becomes faster with a larger defect density. Similar behavior were also observed for low $\mathrm{CO}$ coverages and for $\mathrm{CO}$ diffusion along [001]. These results are very astonishing and puzzling. Intuitively, one would expect $\mathrm{CO}$ diffusion to become slower as the defect density increases since $\mathrm{CO}$ molecules adsorbed on the defect sites have a larger binding energy than those on normal sites and may diffuse slower on one hand and block diffusion of the others on the other hand. Theoretical calculations based on lattice gas models for adsorbate diffusion on an inhomogeneous surface also predict that the diffusion coefficient decreases with increasing inhomogenity ${ }^{3}$. However, both the intuition and the theory have 
assumed that the defect sites has changed only the diffusion activation energy but not the preexponential factor. The probable existence of a compensation effect between the activation energy and the preexponential factor, namely the larger the activation energy the larger the preexponential factor, may lead to faster diffusion on defect sites. However, this does not explain our observation either because the relative low defect density compared to normal site density indicates that the $\mathrm{CO}$ diffusion on surfaces with low density of defects should correspond to normal-site diffusion. The observed invariance of the $\mathrm{CO}$ diffusion activation energy is a further support to this assertion.

No similar study has been carried out on any other systems and a general conclusion is very difficult to reach at this moment. It can be shown (Appendix) that in the usual lattice gas models, even assuming that the interactions between $\mathrm{CO}$ molecules depends on whether they are adsorbed on normal sites or defect sites, an appreciable change in the diffusion coefficient is not possible. Therefore, new mechanism has to be responsible for our observation.

One possible mechanism is long jumps over multiple lattice distance initiated by $\mathrm{CO}$ filled vacancies. As an experimental fact, it is known that a second layer $\mathrm{CO}$ molecule cannot be formed on top of the chemical adsorbed first $\mathrm{CO}$ layer. Therefore, it is very possible that a $\mathrm{CO}$ adsorbed in a vacancy can smooth the potential at that site for other $\mathrm{CO}$. Unlike $\mathrm{CO}$ adsorbed on normal site, a CO molecule adsorbed in a vacancy even geometrically may be less effective in blocking motion of other $\mathrm{CO}$ molecules due to the lowered latitude. As a result, a $\mathrm{CO}$ molecule adsorbed on a normal site near the vacancy can jump across this vacancy and land at the next normal site. Such long jumps can increase the diffusion coefficient through its quadratic dependence on the average jumping length but will not change the diffusion 
activation energy. As long as enough $\mathrm{CO}$ molecules are there on the surface to fill the vacancies, no coverage dependence should be expected for $\mathrm{CO}$ diffusion on surfaces with defects. Therefore, all our results have been consistently explained. The details for this model to work certainly depend on the exact surface morphology. There are experimental evidence that both $\mathrm{Ni}$ adatoms and vacancies can exist on a sputtered $\mathrm{Ni}(110)$ surface. Furthermore, 'a vacancy clustering model has been proposed to interpret the low temperature $(\sim 340 \mathrm{~K})$ thermal annealing results ${ }^{17}$. With this larger vacancy structure, the average distance of long jumps can be much longer. Then, to explain our observation, only a low density of vacancies is needed.

\section{c) CO Diffusion on Stepped Surface}

Study of CO diffusion has also been carried out on a stepped Ni(110) surface. The sample we used has a step density of $\sim 1$ step/40 terrace atoms and a step direction parallel to the [001] direction. In Fig. 2, we have plotted the CO diffusion coefficient along [1 $1 \overline{1} 0]$ as a function of reciprocal temperature for the stepped surface (a) and the good surface (b) at $\theta=0.98$. It is seen that the CO diffusion activation energy on the stepped surface is much higher than that on the good surface $(5 \mathrm{kcal} / \mathrm{mol} \mathrm{vs}$. $2 \mathrm{kcal} / \mathrm{mol})$. This is a clear evidence that diffusion along [110] has been affected by steps. However, detailed understanding is not straight forward. From the discussion in Chapter II, with the interaction between the steps and the terrace neglected the diffusion coefficient on a surface with steps is given by 


$$
\frac{1}{D}=\frac{1}{D_{s}{ }^{\prime}}+\frac{1}{D_{t}{ }^{\prime}}
$$

with $D_{S}^{\prime}$ denoting the diffusion coefficient when the duration time of the adparticle on terraces is negligible and $D_{t}^{\prime}$ denoting the diffusion coefficient when the duration time of the adparticle on steps is negligible respectively. The overall diffusion coefficient $D$ is expected from the above relation to be smaller than the terrace diffusion coefficient $D_{t}{ }^{\prime}\left(\right.$ By definition, $D_{s}{ }^{\prime}<D_{t}{ }^{\prime}$ ). Opposite to this anticipation, the diffusion data shown in Fig. 2 showed diffusion on stepped surface is faster than that on good surface in the high temperature region. Without knowing the true morphology of such a stepped surface we cannot pin down the reason for the observed phenomenon. However, the results can be easily understood if we assume that a significant large number of point defects also exist on the terrace region of the stepped surface.

The coverage dependent $\mathrm{CO}$ diffusion activation energies and the preexponential factors on the stepped surface are shown in Fig. 3. The activation energy of $\mathrm{CO}$ diffusion along [001] direction (parallel to the steps) is not affected by the steps as compared with that on a good surface (Fig. 4 in Chapter V). However, the preexponential factors are larger than that on a good surface. In the [1ํㅣ direction (perpendicular to the steps), both the CO diffusion activation energy and the preexponential factor are affected by the steps significantly. From the discussion in Chapter II, the invariance of $\mathrm{CO}$ diffusion activation energy along [001] on the stepped $\mathrm{Ni}(110)$ is a consequence of the relatively low step density and the preexponential factor change is a consequence of possible presence of point defects associated on the stepped surface. For diffusion perpendicular to the steps, i.e., along [1 $\overline{1} 0]$, the 
difference in the activation energies associated with terrace sites and step sites is very important. Using Eq. (28) of Chapter II,

$$
\gamma=N^{2} \exp \left[\left(E_{\text {diff }}(\text { terrace })-E_{\text {diff }}(\text { step })\right) / k_{B} T\right] \ll 1,
$$

as the condition for steps to dominate diffusion, we find that $N \leq 70$ if the energy difference $E_{\text {diff }}$ (terrace) $-E_{\text {diff }}$ (step) is taken as $\sim 3 \mathrm{kcal} / \mathrm{mol}$. That $N \sim 40$ on the stepped surface we used is in agreement with the step dominated diffusion picture.

In summary, we have reported a $\mathrm{CO}$ diffusion study on $\mathrm{Ni}(110)$ to observe the effects of impurities and defects. The results on $S$ impurities can be understood through surface modification induced by adsorption of $S$. The defect effect on $\mathrm{CO}$ diffusion is more difficult to understand: first, we do not have a good description of defects and defect densities on Ni(110); and second, an intuitive thinking leads to results opposite to observations. We propose a vacancy-filled model that lead to long jumps in diffusion to explain the observations. Results on the effect of steps on $\mathrm{CO} / \mathrm{Ni}(110)$ show that steps can dominate diffusion if they are perpendicular to the diffusion direction. 


\section{Appendix}

In order to show that the observed defect effect on $\mathrm{CO}$ diffusion on $\mathrm{Ni}(110)$ cannot be a thermodynamic result, we use the expression for coverage-dependent diffusion coefficient from Chapter II

$$
D(\theta)=v a^{2} S(\theta) \exp \left(-E_{a} / k_{B} T\right) \exp \left(\mu(\theta) / k_{B} T\right) \frac{1}{\theta}\left(\frac{\partial\left(\mu / k_{B} T\right)}{\partial \ln \theta}\right) T .
$$

where $v$ is the trial frequency, $a$ the lattice constant, $E_{a}$ the activation energy at zero coverage. The quantity $S(\theta)$ and chemical potential $\mu(\theta)$ will be discussed in this appendix. Now we have two kind of sites, normal sites and defect sites (ignoring the differences among adatom sites, vacancy site, and double vacancy sites, and so on) on the $\mathrm{Ni}(110)$ surface. The adsorption of $\mathrm{CO}$ on these two kinds of sites resulted in different binding energies, $E_{D}$ on defect sites and $E_{N}$ on normal sites. Using

$$
a=\exp \left(E_{N} / k_{B} T\right) \text {, }
$$

and

$$
b=\exp \left(E_{D} / k_{B} T\right)
$$

we can write down the free energy for a noninteracting $\mathrm{CO}$ adlayer

$$
\exp (-\beta F)=Z=\sum_{n=0}^{N_{d}} \frac{N_{d} !}{n !\left(N_{d}-n\right) !} \frac{N_{t} !}{(N-n) !\left(N_{t}-N+n\right) !} a^{N-n_{b} n}
$$


where $N_{t}$ is the number of normal sites, $N_{d}$ the number of defect sites, $N$ the total number of $\mathrm{CO}$ molecules adsorbed on the surface, and $n$ the number of $\mathrm{CO}$ molecules adsorbed on the defect sites. There is no exact way to carry out this summation. However, we can simply take the most probable configuration of $n$ without introducing too much error. Let the firsi order derivative of the quantity within the summation symbol with respect to $n$ be zero, we obtain an equation for the most probable $n$,

$$
\frac{\left(N_{d}-n\right)(N-n)}{n\left(N_{t}-N+n\right)}=\frac{a}{b}
$$

If the interaction between the $\mathrm{CO}$ molecules comes only from nearest neighbors and can be treated as perturbation and the mean field approximation is employed, the free energy for an interacting system cari be expressed as

$$
\begin{aligned}
\exp (-\beta F)=Z=\frac{N_{d} !}{n !\left(N_{d}-n\right) !} \frac{N_{t} !}{(N-n) !\left(N_{t}-N+n\right) !} a^{N-n_{b} n} \\
\quad \exp \left(-N_{t t} \varepsilon_{t t} / k_{B} T\right) \exp \left(-N_{d t} \varepsilon_{d t} / k_{B} T\right)
\end{aligned}
$$

where $N_{t t}$ is the number of pairs of nearest neighbor CO's on the good sites, $\varepsilon_{t t}$ the corresponding CO-CO interaction; $\mathrm{N}_{\mathrm{dt}}$ is the number of pairs of nearest neighbor CO's with one $\mathrm{CO}$ on good site and other on defect site, $\varepsilon_{\mathrm{dt}}$ the corresponding CO-CO interaction. In the mean field approximation(see Ref. 19a in Chapter II),

$$
\begin{aligned}
& N_{t t}=(N-n)^{2} / N_{t} \\
& N_{d t}=(N-n) n / N_{t}
\end{aligned}
$$


The chemical potential will be given by

$$
\begin{aligned}
\mu / k T=\frac{\partial \beta F}{\partial N} & \\
=\left(1-\frac{\partial n}{\partial N}\right) & \ln \frac{N-n}{N_{t}-N+n}-\ln a+\frac{1}{N_{t}}\left[(N-n) \frac{2 \varepsilon_{t t}}{k_{B} T}+\frac{\varepsilon_{d t}}{k_{B} T}\right] \\
& +\frac{1}{N_{t}}\left[(N-2 n) \frac{\varepsilon_{d t}}{k_{B} T}-(N-n) \frac{2 \varepsilon_{t t}}{k_{B} T}\right] \frac{\partial n}{\partial N}
\end{aligned}
$$

Expressing this in terms of coverages, we have

$$
\begin{aligned}
\mu / k T=\left(1+\frac{\partial \theta_{d}}{\partial \theta}\right) & \ln \frac{\theta-\theta_{d}}{\theta_{t}-\theta-\theta_{d}}-\ln a+ \\
& +\frac{1}{\theta_{t}}\left[\left(\theta-\theta_{d}\right) \frac{2 \varepsilon_{t t}}{k_{B} T}+\theta_{d} \frac{\varepsilon_{d t}}{k_{B} T}\right] \\
& +\frac{1}{\theta_{t}}\left[\left(\theta-2 \theta_{d}\right) \frac{\varepsilon_{d t}}{k_{B} T}-\left(\theta-\theta_{d}\right) \frac{2 \varepsilon_{t t}}{k_{B} T}\right] \frac{\partial \theta_{d}}{\partial \theta}
\end{aligned}
$$

where $\Theta_{t}$ is the percentage of the normal sites on the surface, $\theta$ the CO coverage and $\theta_{d}$ the CO coverage on defect sites. The thermodynamic factor $\left(\frac{\partial\left(\mu / k_{B} T\right)}{\partial \ln \theta}\right) T$ can then be found from the above expression and is involved with first and second derivatives of $\theta_{d}$ with respect to $\theta$. The defect associated contribution to the diffusion coefficient is related to $\theta_{d}$ and its derivatives. However, with a reasonable difference in the binding energy for $\mathrm{CO}$ on defect and normal sites, a ratio of 10-1000 can be expected for b/a. In this range, $n$ is almost equal to the defect sites. Therefore, the derivatives of $\theta_{d}$ are very small and their contribution to the diffusion coefficient negligible. Numerical calculations show that $10 \%$ increase in $D$ could be 
expected from $5 \%$ defect on the surface. This is too small to explain our experimental results. 


\section{References}

${ }^{1}$ See a recent review by R. Gomer, Rep. Prog. Phys. 53, 917(1990).

${ }^{2}$ V. P. Zhdanov, Surf. Sci. 194, 1(1988); Phys. Lett. A 137, 225(1989).

${ }^{3}$ C. H. Mak, H. C. Anderson, S. M. George, J. Chem. Phys. 88, 4052(1988); V. Pereyra, G. Zgrablich and V. P. Zhdanov, Langmuir 6, 691(1990); F. Bulnes, J.

L. Riccardo, G. Zgrablich and V. Pereyra, Surf. Sci. 260, 304(1992).

${ }^{4}$ For example, inconsistent results have been obtained by different authors on the following systems: CO/Ni(111), X. D. Zhu, Th. Rasing, and Y. R. Shen, Phys. Rev. Lett. 61, 2883(1988); T. S. Lin, H.-J. Lu, And R. Gomer, Surf. Sci. 234, 251(1990). H/Ni(100), S. M. George, A. M. Desantolo, and R. B. Hall, Surf. Sci. 159, L425(1985); D. A. Mullins, B. Roop, and J. M. White, Chem. Phys. Lett. 129, 511(1986); T.-S. Lin and R. Gomer, Surf. Sci. 255, 41(1991); X. D. Zhu, A. Lee, and A. Wong, Phys. Rev. Lett. 68, 1862(1992). CO/Pt(111), B. Poelsema, L. K. Verherij, and G. Comsa, Phys. Rev. Lett. 49, 1731(1982); J. E. Reutt-Robey, D. J. Doren, Y. J. Chabal, and S. B. Christman, Phys. Rev. Lett. 61, 2778(1988); V. J. Kwasniewski, and L. D. Schmidt, Surf. Sci. 274, 329(1992).

${ }^{5}$ M. C. Tringides, J. Chem. Phys. 92, 2077(1990); M. C. Tringides and R. Gomer, Surf. Sci. 265, 283(1992).

${ }^{6}$ W. Daum, Surf. Sci. 182, 521(1987).

${ }^{7}$ M. Blaszczyszyn, R. Blaszczyszyn, R. Meclewski, A. J. Melmed and T. E. Madey, Surf. Sci. 131, 433(1983).

${ }^{8}$ Xu-dong Xiao, Yuanlin Xie and Y. R. Shen, Surf. Sci. 271, 295(1992).

${ }^{9}$ R. A. Dalla Betta, A. G. Piken and M. Shelef, J. Catalysis 40, 173(1975); C. H. Bartholomew, G. D. Weatherbee and G. A. Jarvi, J. Catalysis 60, 257(1979); 
M. A. Vannice, Catalysis Rev. Sci. Eng. 14, 153(1976); R. D. Kelley and D. W. Goodman, in: Chemical Physics of Solid Surfaces and Heterogeneous

Catalysis, Vol. 4, Eds. D. A. King and D. P. Woodruff (Elsevier, Amsterdam, $1982)$ p427.

${ }^{10}$ M. P. Kiskinova, Surf. Sci. Rep. 8, 359(1988).

11 D. R. Warburton, G. Thornton, D. Norman, C. H. Richardson and R. Mcgrath, Surf. Sci. 189/190, 495(1987); R. Baudoing, E. Blanc, C. Gaubert and Y. Gauthier, Surf. Sci. 128, 22(1983).

${ }^{12}$ M. W. Finnis, and V. Heine, J. Phys. F4, L37(1974).

${ }^{13}$ W. Oed, U. Starke, K. Heinz, K. Muller and J. B. Pendry, Surt. Sci. 251/252, 488(1991).

${ }^{14}$ R. J. Madix, Michael Thornburg and S.-B. Lee, Surf. Sci. 133, L447(1983).

15 Maya Kiskinova and D. Wayne Goodman, Surf. Sci. 108, 64(1981).

${ }^{16}$ P. J. Feibelman, Annu. Rev. Phys. Chem. 40, 261(1989); P. J. Feibelman and D. R. Hamann, Phys. rev. Lett. 52, 61(1984); Surf. Sci. 149, 48(1985).

${ }^{17}$ L. K. Verheij, J. A. Van Den Berg and D. G. Armour, Surf. Sci. 122, 216(1982).

18 J. D. Weeks and G. H. Gilmer, Adv. Chem. Phys. 40, 157(1979).

${ }^{19}$ For examples, S. M. Yalisove, W. R. Graham, E. D. Adams, M. Copel and T. Gustafsson, Surf. Sci. 171, 400(1986); B. Voigtander, D. Bruchmann, S. Lehwald and H. Ibach, Surf. Sci. 225, 151(1990); D. R. Huntley, Surf. Sci. 240, 13(1990).

20 I. K. Robinson, E. Vlieg, and K. Kern, Phys. Rev. Lett. 63, 2578(1989).

${ }^{21}$ Klaus Kern, Ian K. Robinson and Elias Vlieg, Surf. Sci. 261, 118(1992).

22 E. H. Conrad, Prog. Surf. Sci. 39, 65(1992); Y. Cao, and E. H. Conrad, Phys. Rev. Lett. 64, 447(1990). 
${ }^{23}$ I. M. Lifshitz, Sov. Phys. JETP 15, 939(1962); S. M. Allen and J. W. Cahn, Acta Metall. 27, 1085(1979).

${ }^{24}$ H. P. Bonzel and E. E. Latta, Surf. Sci. 76, 275(1978).

25 D. W. Basset and P. R. Webber, Surf. Sci. 70, 520(1978). 


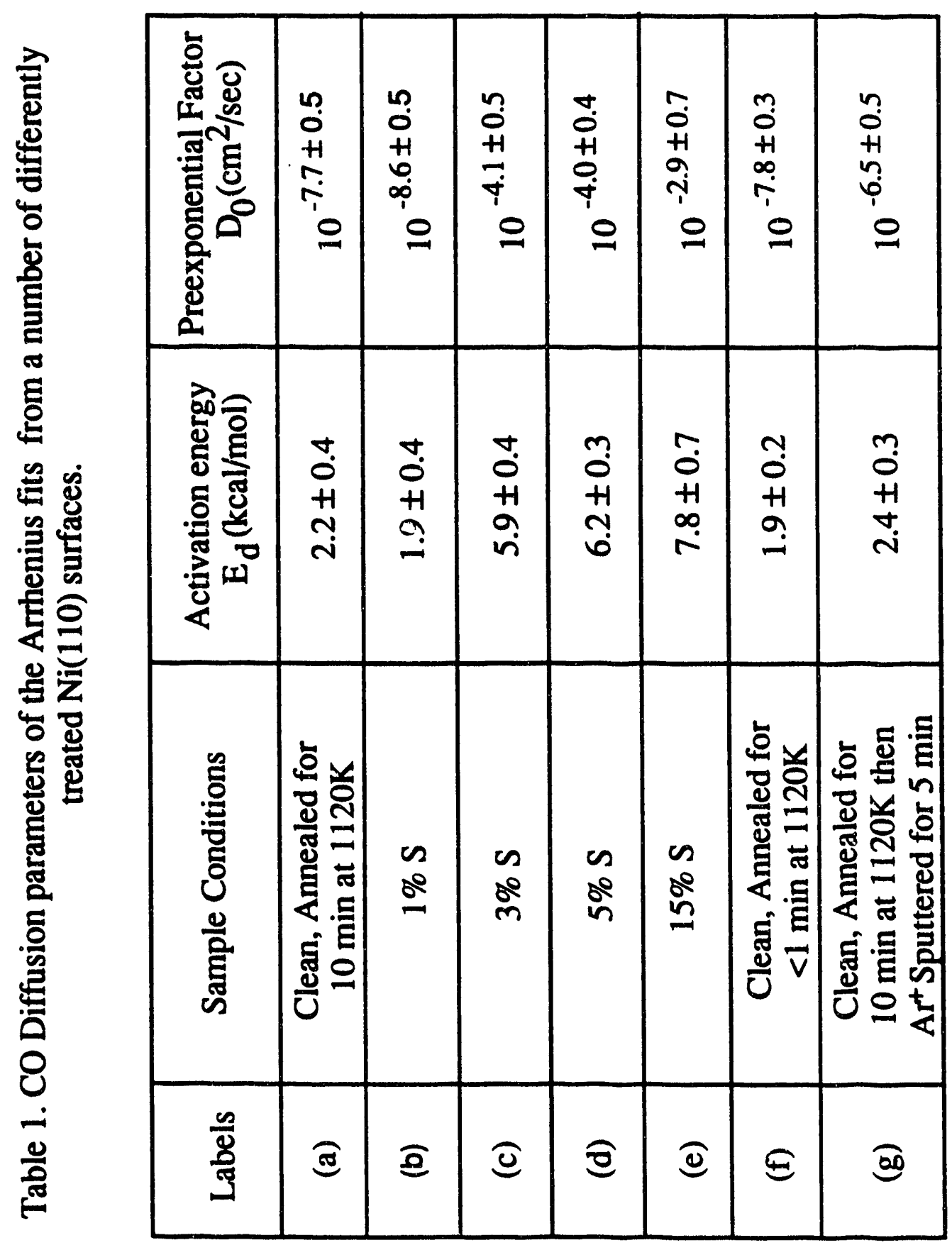




\section{Figure Captions}

Figure 1: CO Diffusion coefficient along [170] as a function of reciprocal temperature for a number of differently treated $\mathrm{Ni}(110)$ surfaces at coverage $\theta=0.98$ : (a) normally prepared, (b) $1 \% \mathrm{~S}$, (c) $3 \% \mathrm{~S}$, (d) $5 \% \mathrm{~S}$, (e) $15 \% \mathrm{~S}$ contaminated, $(f)$ flash-annealed, $(g)$ normally prepared followed by $5 \mathrm{~min} \mathrm{Ar}^{+}$ sputtering.

Figure 2: CO diffusion coefficient along [110] as a function of reciprocal temperature at coverage $\theta=0.98$ on (a) good surface and (b) stepped surface. Both surfaces have been flash-annealed after 30 minutes $\mathrm{Ar}^{+}$sputtering. The stepped surface has a step density of 1 step/40 terrace atoms and a direction

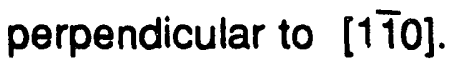

Figure 3: Diffusion: activation energies and preexponential factors as a function of coverage along (a) [1] ] and (b) [001] for $\mathrm{CO}$ on the stepped $\mathrm{Ni}(110)$ surface. 


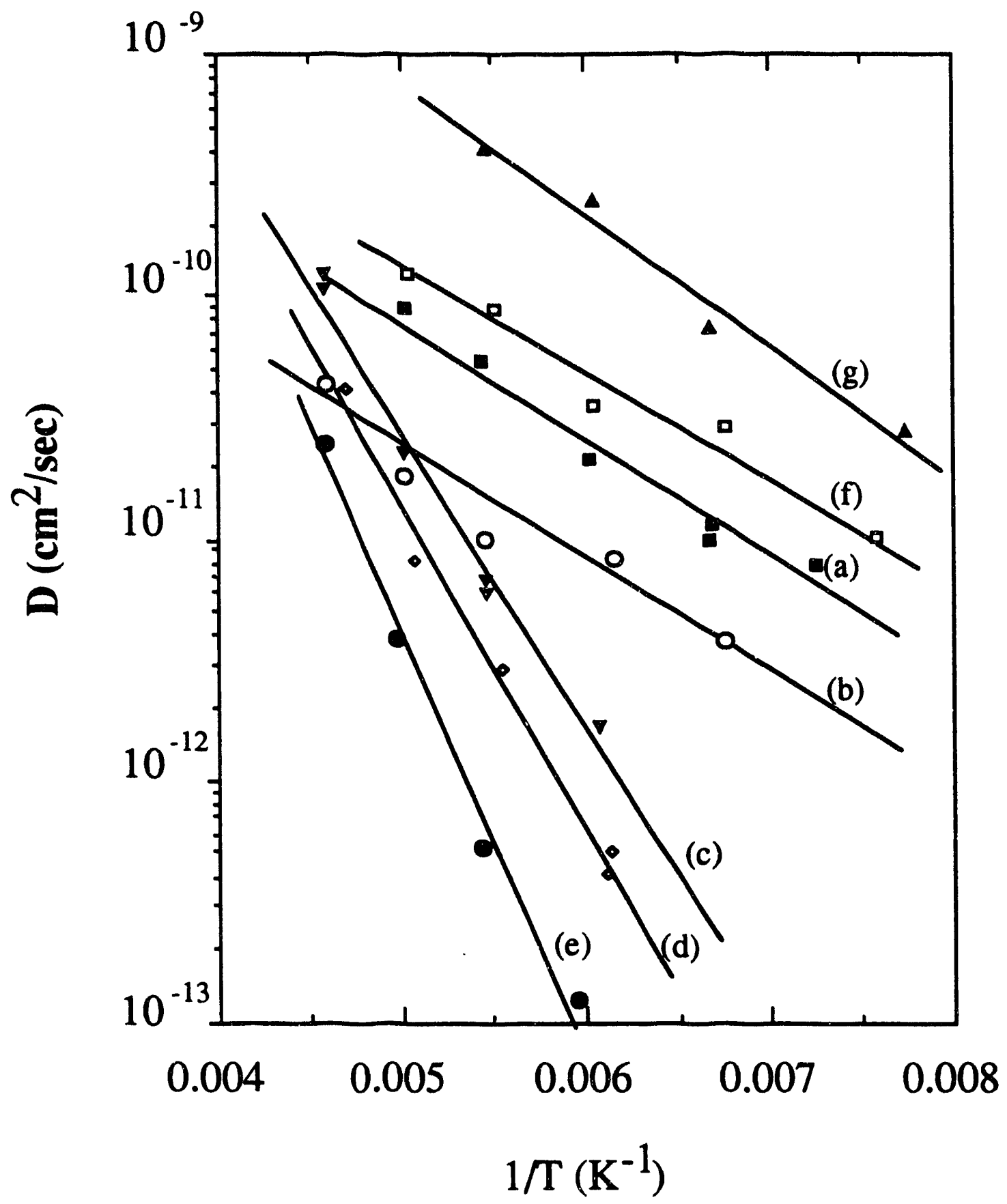

Figure 1 


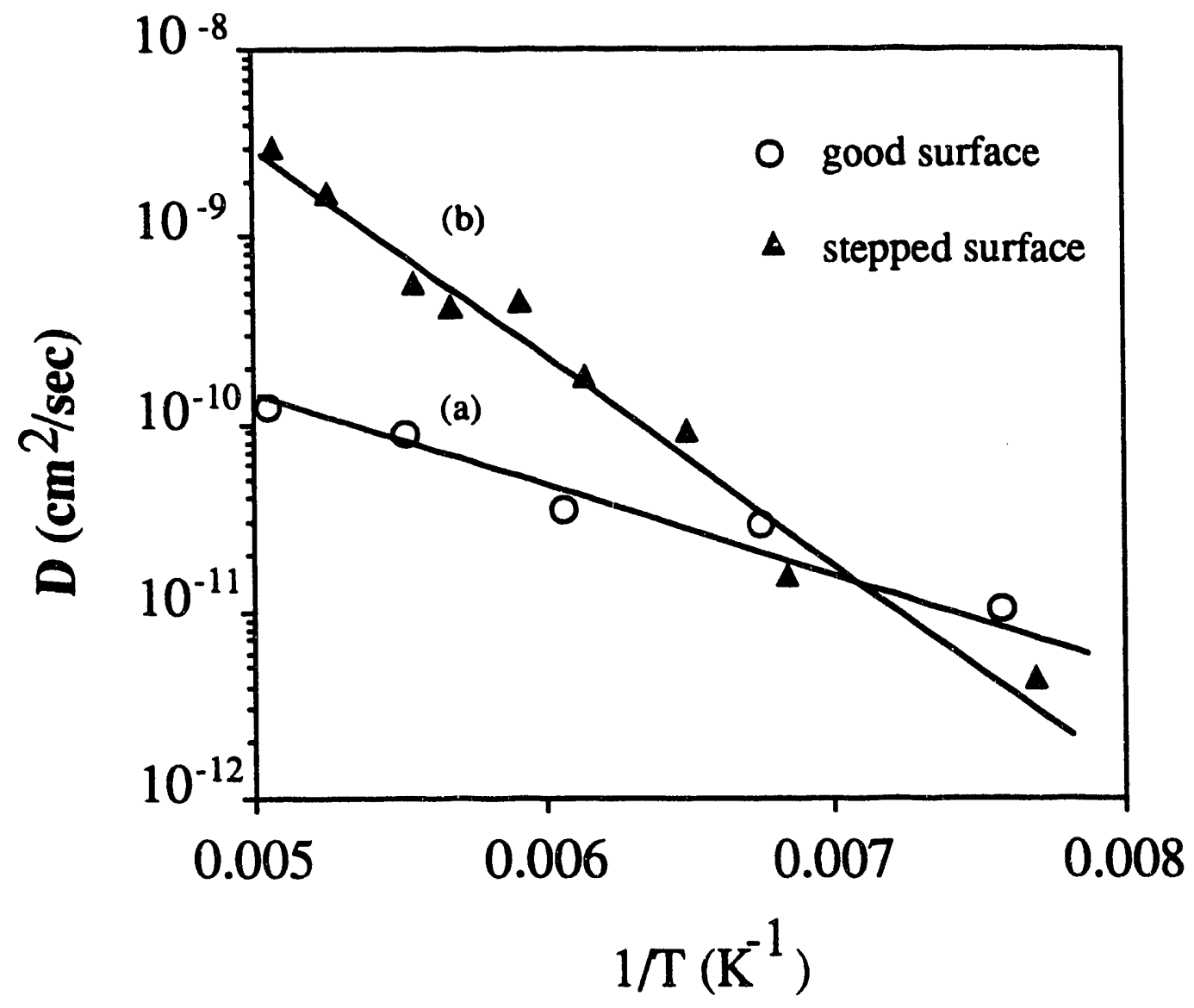

Figure 2 


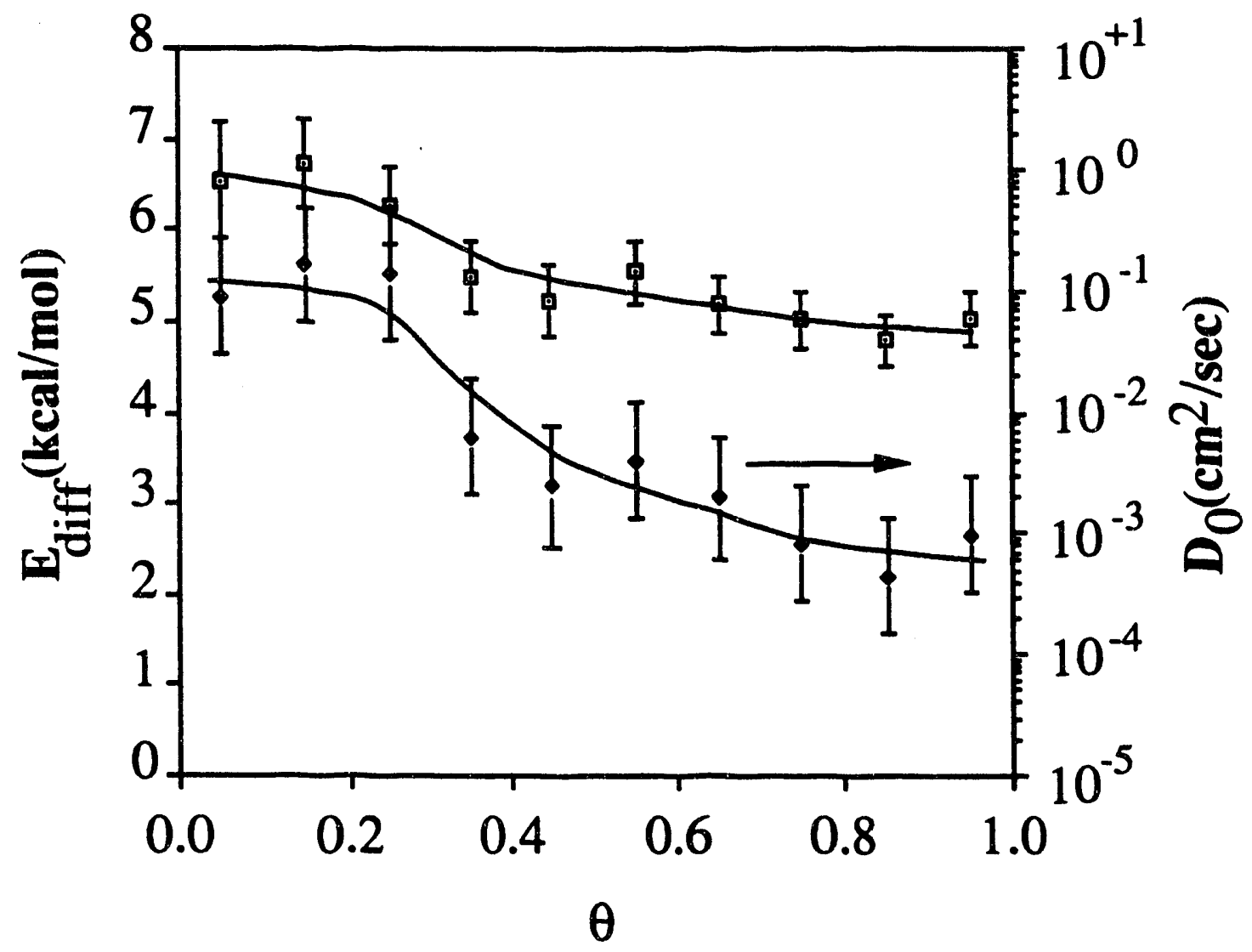

Figure 3(a) 


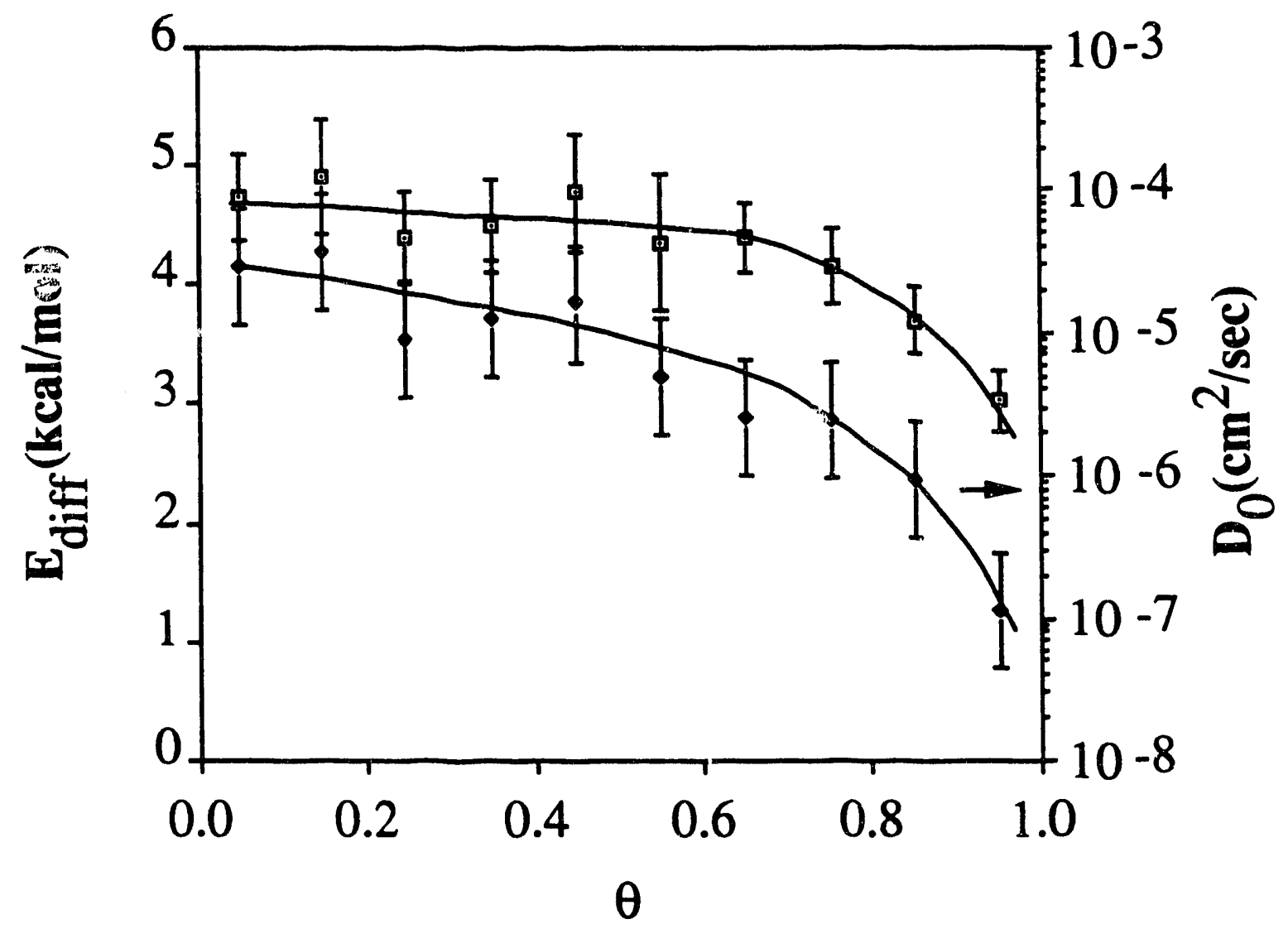

Figure 3(b) 


\section{Future Prospects}

In the previous chapters many aspects of surface diffusion have been ciscussed. However, surface diffusion is not yet a mature field and there are still many problems to solve. The importani issues in surface diffusion studies are: First, the existing data measured by different methods often do not overlap due to their limited dynamic ranges and are often contradictory. The optical diffraction method presented in this thesis with its extremely large dynamic range should contribute in resolving this problem to a great extent. Second, the systems that have been measured are often limited to adsorbate diffusion on refractory metal surfaces. Diffusion on soft metal surfaces and semiconductor surfaces should be explored complementarily. Third, theoretical studies of surface diffusion are very limited, especially for chemical diffusion. Models that can account for the adsorbate-adsorbate interaction in more sophisticated manner than the lattice gas model must be developed. The role of the thermodynamic factor $\left(\frac{\partial\left(\mu / k_{B} T\right)}{\partial \ln \theta}\right) T$ in surface diffusion in connection with adlayer properties has to be further invesiigated. Only with substantial advance in theory, can a variety of experimental results be understood.

Related with the first issue, the study of effects of defects and impurities presented in Chapter VI has revealed the importance of surface characterization. Since diffusion is very sensitive to the fine conditions of a surface, caution has to be taker, when one compares diffusion data for the nominally same surfaces. This may have contributed to the divergence of the existing data in a large degree.

With the S impurity effect on CO diffusion interpreted in terms of $S$ induced surface structure change in Chapter VI, one may wonder whether impurities of oxygen, and hydrogen can have similar effect. Experiment to 
reveal the answer for it is currently undergoing in Shen's group. Oxygen has a strong interaction with $\mathrm{Ni}(110)$ and presumably will change the structure of $\mathrm{Ni}(110)$ to certain extent. On the other hand, hydrogen interacts with $\mathrm{Ni}(110)$ less stronger and probably will not affect $\mathrm{CO}$ diffusion much.

The observed effect of defects for $\mathrm{CO}$ diffusion on $\mathrm{Ni}(110)$ is counterintuitive and can be explained in terms of multiple lattice distance jump assisted by $\mathrm{CO}$ filled vacancies. Is this picture general? Experiment on other systems, for example, CO/Pt(110) which resembles $\mathrm{CO} / \mathrm{Ni}(110)$ in many aspects, can be used to test our proposed model. Along this direction, significant effort in both experiment and theory has to be invested.

Coverage dependence measurements of $\mathrm{CO}$ diffusion on low Miller index $\mathrm{Ni}$ surfaces have not reached a consistent picture yet. $\mathrm{A}$ measurement for CO/Ni(100) with the optical diffraction technique would be helpful in order to eliminate the possible artifacts due to "hole burning" LITD measurement scheme, which is ill defined for coverage dependence studies. Hopefully the new results will support the conclusion from the other two surfaces, namely long range $\mathrm{CO}-\mathrm{CO}$ interaction does not change the diffusion activation energy. Whether it is generally true that long range interaction does not affect diffusion, systems with other adsorbate and other surfaces should be also investigated. Only with an accumulation of experimental data, can a consistent picture then be built.

Diffusion of adsorbates on semiconductor surfaces are ve:y interesting due to the intrinsic bonding difference between semiconductor surface and metal surface. The covalent bonding in the case of semiconductor presumably provides a much higher potential barrier for adsorbate to overcome in the path of jumping from one well to another. Diffusion of $H$ and $K$ on $\operatorname{GaAs}(110)$ is being investigated with the optical diffraction technique. Since the surface 
potentials of these two systems have been calculated, direct comparison with experimental results will be possible. In particular, diffusion of $K$ could be very interesting because $\mathrm{K}$ might form chains along [1 $\left.{ }^{1} \overline{0}\right]$ on the GaAs(110) surface. Whether $\mathrm{K}$ diffuses as a chain or an atom should be an important issue to address. Even if the diffusion occurs via single atom motion, how the $\mathrm{K}$ atom detach from one chain and jump over some distance to attach to another chain should enrich our understandings on crystal growth. The anisotropy of the systems again provides us one more degree of freedom.

The last, the optical technique for coverage dependence measurement could be improved with a scheme of simultaneous detection of multi-order diffractions as described in Chapter III. A success in implementing such a scheme could save tremendous time and improve the measurement accuracy. 

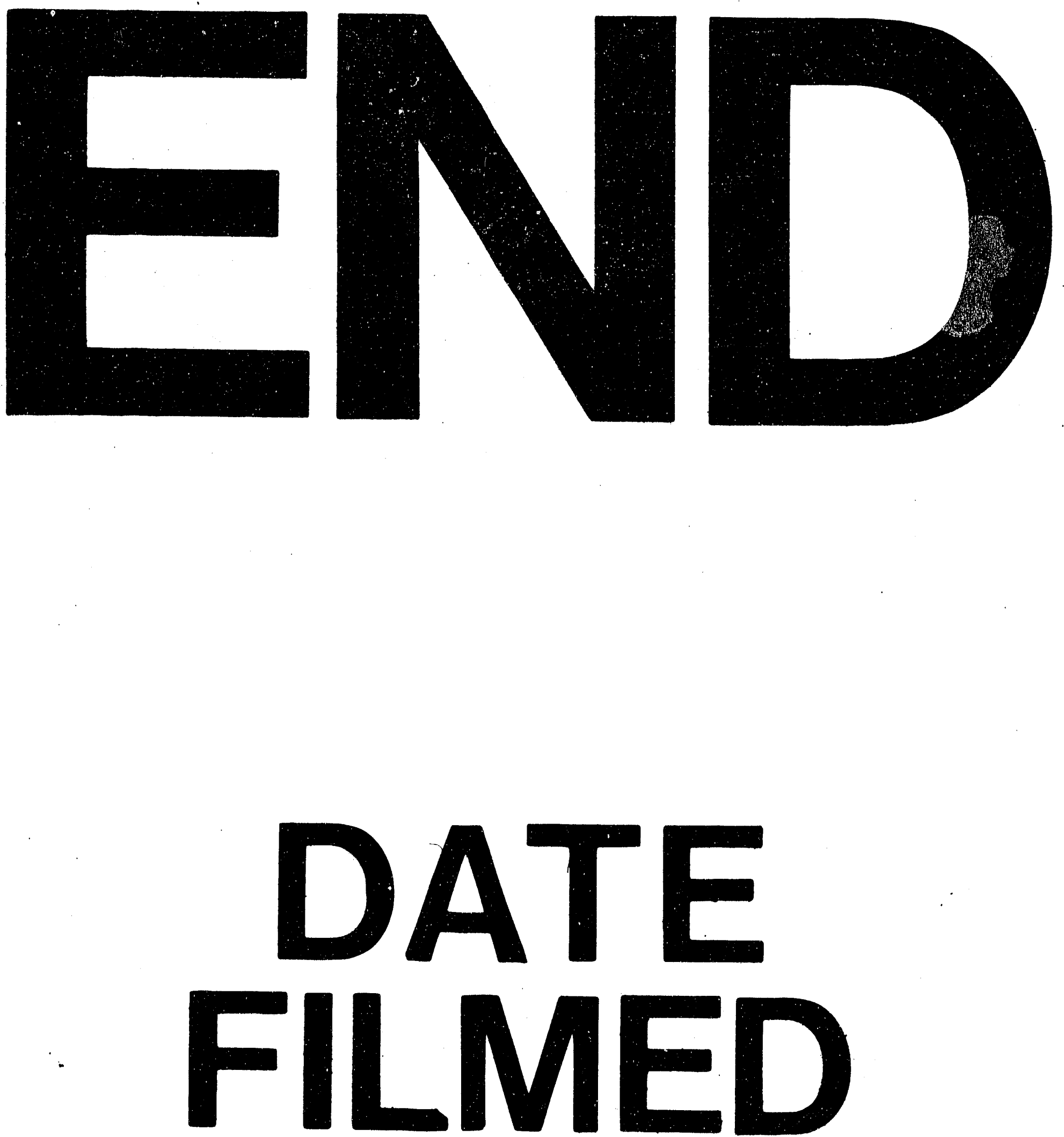

4

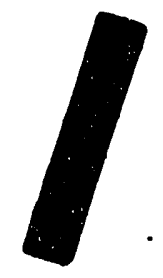

9

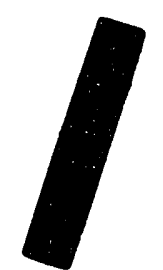

93 
